\title{
Distribuições de Probabilidade no Intervalo Unitário
}

Francimário Alves de Lima

\section{DisSERTAÇÃO APRESENTADA}

$\mathrm{AO}$

Instituto DE MATEMÁTICA E EstatísticA

DA

Universidade DE SÃo PAUlo

PARA

OBTENÇÃO DO TÍTULO

$\mathrm{DE}$

MESTRE EM CIÊNCIA

\section{Programa: Pós-Graduação em Estatística \\ Orientadora: Prof ${ }^{\mathrm{a}}$. Dr ${ }^{\mathrm{a}}$. Silvia Lopes de Paula Ferrari}

Durante o desenvolvimento deste trabalho o autor recebeu auxílio financeiro do CNPq.

São Paulo, março de 2018 


\section{Distribuições de Probabilidade no Intervalo Unitário}

Esta versão da dissertação contém as correções e alterações sugeridas pela Comissão Julgadora durante a defesa da versão original do trabalho, realizada em 16/03/2018. Uma cópia da versão original está disponível no

Instituto de Matemática e Estatística da Universidade de São Paulo.

Comissão Julgadora:

- Prof ${ }^{a}$. Dra ${ }^{a}$ Silvia Lopes de Paula Ferrari - IME-USP

- Prof. Dr. Fábio Mariano Bayer - DE-UFSM

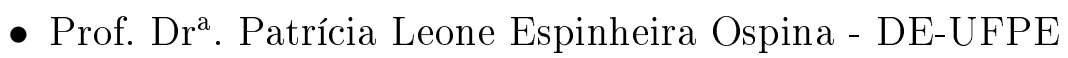




\section{Agradecimentos}

A Deus por tudo e pelo dom da vida.

Aos meus pais Francisco Assis Lima e Maria das Graças Alves e meu irmão Françoaldo Alves de Lima por todo amor.

À professora Silvia Lopes de Paula Ferrari por todos os ensinamentos, que vão além do mundo acadêmico, pela paciência e carinho, e pelo exemplo de pessoa.

A todos os professores e amigos que diretamente e indiretamente influenciaram na minha jornada. Em especial ao meu amigo de infância Cleidson Silva, vulgo Diego, por transformar a vida das pessoas e meus primos-irmãos júnior e Bruno. 


\section{Resumo}

LIMA, Francimário A. de Distribuições de Probabilidade no Intervalo Unitário. 2018. Dissertação (Mestrado) - Instituto de Matemática e Estatística, Universidade de São Paulo, São Paulo, 2018.

A distribuição beta é a mais frequentemente utilizada para a modelagem de dados contínuos observados no intervalo unitário, como taxas e proporções. Embora seja flexível, admitindo formas variadas, tais como J, J invertido, U e unimodal, não é adequada em todas as situações práticas. Nesta dissertação fazemos uma revisão sobre distribuições contínuas no intervalo unitário englobando as distribuições beta, Kumaraswamy, simplex, gama unitária e beta retangular. Também abordamos uma ampla classe de distribuições obtida por transformações (Smithson e Merkle, 2013). Em particular, focamos em duas subclasses, uma apresentada e estudada por Lemonte e Bazán (2015), que chamaremos de classe de distribuições logito, e outra que chamaremos de classe de distribuições logito skew. Todas as distribuições consideradas são aplicadas a conjuntos de dados do Banco Mundial.

Palavras-chave: Banco Mundial, distribuição beta, distribuições contínuas no intervalo unitário. 


\section{Abstract}

Lima, Francimário A. de Probability Distributions in the Unit Interval. 2010. Dissertation (Mesters) - Institute of Mathematics and Statistics, University of São Paulo, São Paulo, 2018.

The beta distribution is the most frequently used for modeling continuous data observed in the unit interval, such as rates and proportions. Although flexible, assuming varied forms, such as J, inverted $\mathrm{J}, \mathrm{U}$ and unimodal, it is not suitable in all practical situations. In this dissertation we make a review on continuous distributions in the unit interval encompassing the beta, Kumaraswamy, simplex, unit gamma and rectangular beta distributions. We also address a wide class of distributions obtained by transformations (Smithson and Merkle, 2013). In particular, we focus on two subclasses, one presented and studied by Lemonte and Bazán (2015), which we will call the logit class of distributions, and another that we will call the logit class of skew distributions. All distributions considered are applied to World Bank data sets.

Keywords: World Bank, beta distribution, continuous distributions in the unit interval. 


\section{Sumário}

Lista de Abreviaturas $\quad$ ix

Lista de Símbolos $\quad$ xi

Lista de Figuras $\quad$ xiii

Lista de Tabelas $\quad$ xvii

1 Introdução $\quad 1$

1.1 Organização da dissertação . . . . . . . . . . . . . . . . . 2

2 Principais distribuições no intervalo unitário $\quad 3$

3 Distribuições no intervalo unitário geradas por transformações 11

3.1 Introdução . . . . . . . . . . . . . . . . . . . . . . . 11

3.2 Transformação logito . . . . . . . . . . . . . . . . . 12

3.2 .1 Classe logito . . . . . . . . . . . . . . 15

3.2 .2 Classe logito skew . . . . . . . . . . . . . . . 19

3.3 Estimação . . . . . . . . . . . . . . . . . . . 23

3.3 .1 Estimação na classe logito . . . . . . . . . . . . . . . 24

3.3.2 Estimação na classe logito skew . . . . . . . . . . . . 25

4 Aplicação $\quad 27$

5 Considerações Finais $\quad 51$

$\begin{array}{ll}\text { Referências Bibliográficas } & 53\end{array}$ 
viii SUMÁRIO 


\section{Lista de Abreviaturas}

fdp Função densidade de probabilidade

fda Função distribuição acumulada

v.a. Variável aleatória

i.i.d. Independente e identicamente distribuído

AIC Critério de informação de Akaike

BIC Critério de informação bayesiana

TRGV Teste da razão de verossimilhanças generalizado 


\title{
Lista de Símbolos
}

\author{
$\Phi_{X}(t) \quad$ fdp da v.a. $X$ no ponto $t$ \\ $\Gamma(t) \quad$ função gama no ponto $t$ \\ $\Gamma(t ; x) \quad$ função gama incompleta com limite $x$ no ponto $t$ \\ $\Delta_{\mathrm{m}} \quad$ Evidência empírica de escala de Burnham e Anderson \\ $R M_{\mathrm{m}} \quad$ Razão da evidência \\ $w_{\mathrm{m}} \quad$ Peso do modelo \\ $V_{\mathrm{RV}} \quad$ Razão de verossimilhanças generalizada \\ $\mathrm{B}(x, y) \quad$ Função beta no ponto $(x, y), x>0, y>0$ \\ ${ }_{2} F_{1}(a ; b ; c ; d)$ Função hipergeométrica no ponto $(a, b, c, d)$
}


xii LISTA DE SÍMBOLOS 


\section{Lista de Figuras}

2.1 Funções densidade de probabilidade da distribuição beta $(\mu, \phi):(\mathrm{a})(\mu, \phi)=$ $(0.5,1),(\mathrm{b})(\mu, \phi)=(0.5,2),(\mathrm{c})(\mu, \phi)=(0.6,2.2),(\mathrm{d})(\mu, \phi)=(0.5,10),(\mathrm{e})$ $(\mu, \phi)=(0.1,5),(\mathrm{f})(\mu, \phi)=(0.9,5),(\mathrm{g})(\mu, \phi)=(0.2,10)$ e $(\mathrm{h})(\mu, \phi)=(0.8,10) 5$

2.2 Funções densidade de probabilidade da distribuição $\operatorname{Kuma}(\mu, \phi):(\mathrm{a})(\mu, \phi)=$ $(0.5,0.5),(\mathrm{b})(\mu, \phi)=(0.5,1),(\mathrm{c})(\mu, \phi)=(0.5,3),(\mathrm{d})(\mu, \phi)=(0.5,8),(\mathrm{e})$ $(\mu, \phi)=(0.25,3),(\mathrm{f})(\mu, \phi)=(0.65,1),(\mathrm{g})(\mu, \phi)=(0.75,3)$ e $(\mathrm{h})(\mu, \phi)=$

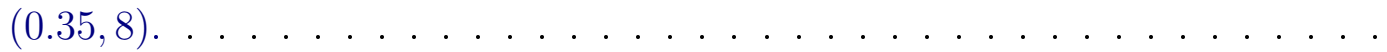

2.3 Funções densidade de probabilidade da distribuição $\mathrm{S}(\mu, \phi):(\mathrm{a})(\mu, \phi)=$ $(0.5, .05),(\mathrm{b})(\mu, \phi)=(0.5,0.3),(\mathrm{c})(\mu, \phi)=(0.75,1),(\mathrm{d})(\mu, \phi)=(0.25,1)$, (e) $(\mu, \phi)=(0.75,0.05)$, (f) $(\mu, \phi)=(0.25,0.05)$, (g) $(\mu, \phi)=(0.6,0.25)$ e $(\mathrm{h})$ $(\mu, \phi)=(0.4,0.1) \ldots \ldots \ldots \ldots \ldots \ldots \ldots \ldots \ldots \ldots \ldots \ldots \ldots \ldots \ldots$

2.4 Funções densidade de probabilidade da distribuição $\operatorname{Gu}(\mu, \phi):(a)(\mu, \phi)=$ $(0.5,0.5),(\mathrm{b})(\mu, \phi)=(0.5,1),(\mathrm{c})(\mu, \phi)=(0.5,5),(\mathrm{d})(\mu, \phi)=(0.05,9),(\mathrm{e})$ $(\mu, \phi)=(0.95,9),(\mathrm{f})(\mu, \phi)=(0.8,5),(\mathrm{g})(\mu, \phi)=(0.2,5)$ e $(\mathrm{h})(\mu, \phi)=$

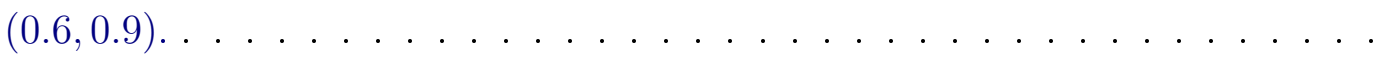

2.5 Funções densidade de probabilidade da distribuição $\operatorname{BR}(\gamma, \phi, \alpha):(\mathrm{a})(\gamma, \phi, \alpha)=$ $(0.5,2,0.7),(\mathrm{b})(\gamma, \phi, \alpha)=(0.5,1,0.3),(\mathrm{c})(\gamma, \phi, \alpha)=(0.5,7,0.4),(\mathrm{d})(\gamma, \phi, \alpha)=$ $(0.2,10,0.6),(\mathrm{e})(\gamma, \phi, \alpha)=(0.8,10,0.2),(\mathrm{f})(\gamma, \phi, \alpha)=(0.3,5,0.2),(\mathrm{g})(\gamma, \phi, \alpha)=$ $(0.3,5,0.8)$ e $(\mathrm{h})(\gamma, \phi, \alpha)=(0.75,5,0.5) \ldots \ldots \ldots \ldots$

3.1 Funções densidade de probabilidade da distribuição $\operatorname{LL}(\mu, \phi):(\mathrm{a})(\mu, \phi)=$ $(0,0.1),(\mathrm{b})(\mu, \phi)=(0,1),(\mathrm{c})(\mu, \phi)=(0,2),(\mathrm{d})(\mu, \phi)=(0,10),(\mathrm{e})(\mu, \phi)=$ $(-3,1),(\mathrm{f})(\mu, \phi)=(3,1),(\mathrm{g})(\mu, \phi)=(-1.5,4)$ e $(\mathrm{h})(\mu, \phi)=(1.5,4) \ldots$.

3.2 Funções densidade de probabilidade da distribuição $\operatorname{LN}(\mu, \phi):($ a) $(\mu, \phi)=$ $(0,0.1),(\mathrm{b})(\mu, \phi)=(0,0.3),(\mathrm{c})(\mu, \phi)=(0,6),(\mathrm{d})(\mu, \phi)=(1,0.3),(\mathrm{e})$ $(\mu, \phi)=(-3.5,1),(\mathrm{f})(\mu, \phi)=(3.5,1),(\mathrm{g})(\mu, \phi)=(-1,4)$ e $(\mathrm{h})(\mu, \phi)=(1,4) .17$

3.3 Funções densidade de probabilidade da distribuição $\operatorname{LT}(\mu, \phi, \nu):(\mathrm{a})(\mu, \phi, \nu)=$ $(0,3,1),(\mathrm{b})(\mu, \phi, \nu)=(0,0.1,4),(\mathrm{c})(\mu, \phi, \nu)=(1.5,1.5,1),(\mathrm{d})(\mu, \phi, \nu)=$ $(-1.5,1.5,1),(\mathrm{e})(\mu, \phi, \nu)=(-4,1.5,4),(\mathrm{f})(\mu, \phi, \nu)=(4,1.5,4),(\mathrm{g})(\mu, \phi, \nu)=$ $(-1.5,4,3)$ e $(\mathrm{h})(\mu, \phi, \nu)=(1.5,4,50) \ldots \ldots \ldots$ 
3.4 Funções densidade de probabilidade da distribuição $\operatorname{LEP}(\mu, \phi, \nu):($ a) $(\mu, \phi, \nu)=$ $(0,0.1,1.5),(\mathrm{b})(\mu, \phi, \nu)=(0,0.1,10),(\mathrm{c})(\mu, \phi, \nu)=(0,3,10),(\mathrm{d})(\mu, \phi, \nu)=$ $(0,6,5),(\mathrm{e})(\mu, \phi, \nu)=(1,3,1),(\mathrm{f})(\mu, \phi, \nu)=(1,3,1.5),(\mathrm{g})(\mu, \phi, \nu)=$ $(-1,3,10)$ e $(\mathrm{h})(\mu, \phi, \nu)=(-1.5,1,10)$.

3.5 Funções densidade de probabilidade da distribuição $\operatorname{LSL}(\mu, \phi, \lambda):(\mathrm{a})(\mu, \phi, \lambda)=$ $(0,10,-10),(\mathrm{b})(\mu, \phi, \lambda)=(1,1.5,-10),(\mathrm{c})(\mu, \phi, \lambda)=(1,1,10),(\mathrm{d})(\mu, \phi, \lambda)=$ $(-1.3,1,4),(\mathrm{e})(\mu, \phi, \lambda)=(-4,1,-10),(\mathrm{f})(\mu, \phi, \lambda)=(4,2,-10),(\mathrm{g})(\mu, \phi, \lambda)=$ $(0,1,0)$ e $(\mathrm{h})(\mu, \phi, \lambda)=(0,5,10)$

3.6 Funções densidade de probabilidade da distribuição $\operatorname{LSN}(\mu, \phi, \lambda):(\mathrm{a})(\mu, \phi, \lambda)=$ $(0,2,10),(\mathrm{b})(\mu, \phi, \lambda)=(0,2,-10),(\mathrm{c})(\mu, \phi, \lambda)=(1,0.3,8),(\mathrm{d})(\mu, \phi, \lambda)=$ $(1,1,5),(\mathrm{e})(\mu, \phi, \lambda)=(-2.1,0.1,3),(\mathrm{f})(\mu, \phi, \lambda)=(-2.5,10,-10),(\mathrm{g})(\mu, \phi, \lambda)=$ $(0,1,0)$ e $(\mathrm{h})(\mu, \phi, \lambda)=(0,5,-5) \ldots \ldots \ldots \ldots \ldots \ldots \ldots \ldots \ldots \ldots \ldots \ldots$

3.7 Funções densidade de probabilidade da distribuição $\operatorname{LST}(\mu, \phi, \nu, \lambda):(\mathrm{a})(\mu, \phi, \nu, \lambda)=$ $(1,2,5,-10),(\mathrm{b})(\mu, \phi, \nu, \lambda)=(1,2,5,10),(\mathrm{c})(\mu, \phi, \nu, \lambda)=(-1.5,0.7,3,-10)$, (d) $(\mu, \phi, \nu, \lambda)=(-1.5,1,0.5,-6),(\mathrm{e})(\mu, \phi, \nu, \lambda)=(0,1,2,-5),(\mathrm{f})(\mu, \phi, \nu, \lambda)=$ $(1,1,6,5),(\mathrm{g})(\mu, \phi, \nu, \lambda)=(0,1,2,0)$ e $(\mathrm{h})(\mu, \phi, \nu, \lambda)=(0,5,6,-5) \ldots \ldots 22$

3.8 Funções densidade de probabilidade da distribuição $\operatorname{LSEP}(\mu, \phi, \nu, \lambda):(\mathrm{a})(\mu, \phi, \nu, \lambda)=$ $(-1,0.5,0.8,-10),(\mathrm{b})(\mu, \phi, \nu, \lambda)=(-1,1,8,10),(\mathrm{c})(\mu, \phi, \nu, \lambda)=(0.4,0.5,1.4,10)$, (d) $(\mu, \phi, \nu, \lambda)=(1,2,0.2,-7.5),(\mathrm{e})(\mu, \phi, \nu, \lambda)=(-1,2.3,1,-10),(\mathrm{f})(\mu, \phi, \nu, \lambda)=$ $(1,1,4,-10),(\mathrm{g})(\mu, \phi, \nu, \lambda)=(0,1,2,0)$ e $(\mathrm{h})(\mu, \phi, \nu, \lambda)=(0,5,2,-5) . \quad$.

4.1 Fdp e fda ajustadas, beta (contínua), Kumaraswamy (tracejada) e BR (pontilhada), suavização e fda empírica (verde); Exportações de alta tecnologia. .

4.2 Fdp e fda ajustadas, beta (contínua), Gu (tracejada) e Kumaraswamy (pontilhada), suavização e fda empírica (verde); Despesas em educação.

4.3 Fdp e fda ajustadas, beta (contínua), Kumaraswamy (tracejada) e Gu (pontilhada), suavização e fda empírica (verde); Terra arável.

4.4 Fdp e fda ajustadas, beta (contínua), simplex (tracejada) e Gu (pontilhada), suavização e fda empírica (verde); Serviços. . . . . . . . . . . . . . . .

4.5 Fdp e fda ajustadas, beta (contínua), Gu (tracejada) e Kumaraswamy (pontilhada), suavização e fda empírica (verde); Despesas em pesquisa e desenvolvimento.

4.6 Fdp e fda ajustadas, beta (contínua), Gu (tracejada) e Kumaraswamy (pontilhada), suavização e fda empírica (verde); Taxa de alfabetização. . . . . . . .

4.7 Fdp e fda ajustadas, beta (contínua), BR (tracejada) e Gu (pontilhada), suavização e fda empírica (verde); Energia alternativa e nuclear. . . . . . . . . .

4.8 Fdp e fda ajustadas, beta (contínua), LSEP (tracejada) e LSL (pontilhada), suavização e fda empírica (verde); Área florestal. . . . . . . . . . . . . . .

4.9 Fdp e fda ajustadas, beta (contínua), LSEP (tracejada) e BR (pontilhada), suavização e fda empírica (verde); Exportações de combustível. . . . . . . . . 
4.10 Fdp e fda ajustadas, beta (contínua), LL (tracejada) e LT (pontilhada), suavização e fda empírica (verde); Despesas. . . . . . . . . . . . . 41

4.11 Fdp e fda ajustadas, beta (contínua), LSL (tracejada) e Kumarwamy (pontilhada), suavização e fda empírica (verde); Terra agrícola. . . . . . . . . . 42

4.12 Fdp e fda ajustadas, beta (contínua), LSL (tracejada) e LST (pontilhada), suavização e fda empírica (verde); População rural. . . . . . . . . . . . . . 43

4.13 Fdp e fda ajustadas, beta (contínua), LSL (tracejada) e LST (pontilhada), suavização e fda empírica (verde); População urbana. . . . . . . . . . . . . . 44

4.14 Fdp e fda ajustadas, beta (contínua), LEP (tracejada) e LSEP (pontilhada), suavização e fda empírica (verde); Saneamento. . . . . . . . . . . 45

4.15 Fdp e fda ajustadas, beta (contínua), LEP (tracejada) e LSEP (pontilhada), suavização e fda empírica (verde); Saneamento urbano. . . . . . . . . . . . 46

4.16 Fdp e fda ajustadas, beta (contínua), LSEP (tracejada) e LST (pontilhada), suavização e fda empírica (verde); Títulos da dívida externa. . . . . . . . . 47 


\section{Lista de Tabelas}

2.1 Algumas características das principais distribuições. . . . . . . . . . . . 10

3.1 Funções $g(u)$ e $W_{g}(u)$ para diferentes distribuições. . . . . . . . . . . . . . 24

4.1 Critérios de seleção de modelos, Exportações de alta tecnologia; $n=123$,

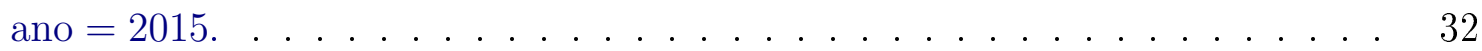

4.2 Medidas descritivas e estimativas, Exportações de alta tecnologia. . . . . . . 32

4.3 Critérios de seleção de modelos, Despesas em educação; $n=83$, ano $=2014$. 33

4.4 Medidas descritivas e estimativas, Despesas em educação. . . . . . . . . . . 33

4.5 Critérios de seleção de modelos, Terra arável; $n=190$, ano $=2014$. . . . . 34

4.6 Medidas descritivas e estimativas, Terra arável. . . . . . . . . . . . . 34

4.7 Critérios de seleção de modelos, Serviços; $n=133$, ano $=2016 \ldots \ldots$. . . . 35

4.8 Medidas descritivas e estimativas, Serviços. . . . . . . . . . . . . 35

4.9 Critérios de seleção de modelos, Despesas em pesquisa e desenvolvimento;

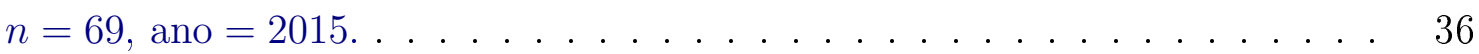

4.10 Medidas descritivas e estimativas, Despesas em pesquisa e desenvolvimento. . 36

4.11 Critérios de seleção de modelos, Taxa de alfabetização; $n=36$, ano $=2014$. . 37

4.12 Medidas descritivas e estimativas, Taxa de alfabetização. . . . . . . . . . . 37

4.13 Critérios de seleção de modelos, Energia alternativa e nuclear; $n=133$, ano $=$

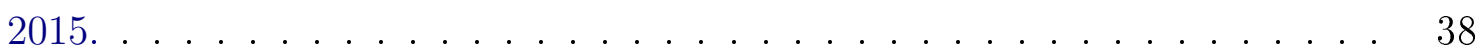

4.14 Medidas descritivas e estimativas, Energia alternativa e nuclear. . . . . . . 38

4.15 Critérios de seleção de modelos, Área florestal; $n=192$, ano $=2015 \ldots$. . . . 39

4.16 Medidas descritivas e estimativas, Área florestal. . . . . . . . . . . . . 39

4.17 Critérios de seleção de modelos, Exportações de combustível; $n=125$, ano $=$

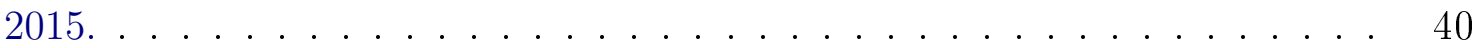

4.18 Medidas descritivas e estimativas, Exportações de combustível. . . . . . . . . 40

4.19 Critérios de seleção de modelos, Despesas; $n=94$, ano $=2015$. . . . . . . 41

4.20 Medidas descritivas e estimativas, Despesas. . . . . . . . . . . . . 41

4.21 Critérios de seleção de modelos, Terra agrícola; $n=192$, ano $=2014$. . . . 42

4.22 Medidas descritivas e estimativas, Terra agrícola. . . . . . . . . . . . 42

4.23 Critérios de seleção de modelos, População rural; $n=193$, ano $=2016$. . . 43

4.24 Medidas descritivas e estimativas, População rural. . . . . . . . . . . . . 43 
4.25 Critérios de seleção de modelos, População urbana; $n=193$, ano $=2016 . \quad$. 44

4.26 Medidas descritivas e estimativas, População urbana. . . . . . . . . . . . 44

4.27 Critérios de seleção de modelos, Saneamento; $n=181$, ano $=2015$. . . . 45

4.28 Medidas descritivas e estimativas, Saneamento. . . . . . . . . . . 45

4.29 Critérios de seleção de modelos, Saneamento urbano; $n=182$, ano $=2015$. $\quad 46$

4.30 Medidas descritivas e estimativas, Saneamento urbano. . . . . . . . . . 46

4.31 Critérios de seleção de modelos, Títulos da dívida externa; $n=110$, ano $=2015$. 47

4.32 Medidas descritivas e estimativas, Títulos da dívida externa. . . . . . . . . . . 47

4.33 Resumo dos ajustes. . . . . . . . . . . . . . . . . 48 


\section{Capítulo 1}

\section{Introdução}

Modelos probabilísticos são utilizados para representar características de uma determina população de interesse. Um dos modelos probabilísticos mais utilizados é o modelo normal, pois além de ter boas propriedades, como, por exemplo, parâmetros interpretáveis, formas amigáveis e fácil tratamento algébrico, está implementado na maioria dos softwares matemáticos e estatísticos e consegue explicar vários fenômenos de diversas áreas do conhecimento. Entretanto, há situações em que o modelo normal não é apropriado, pois não acomoda casos de variáveis assimétricas, de cauda pesada ou com suporte limitado. Modelos mais flexíveis, que conseguem capturar essas características, têm sido estudados. Ley (2015) apresenta uma excelente revisão sobre modelagem flexível e apresenta caminhos para o futuro. Entretanto, todos os modelos probabilísticos que ele apresenta têm como suporte a reta real. Jones (2015) também apresenta famílias de distribuições flexíveis com parâmetro de forma, não necessariamente tendo como suporte a reta real.

A necessidade por avanços e sofisticações nos métodos estatísticos para modelagem mais fidedigna à realidade fez com que esses novos modelos ganhassem importância. Suponha que uma determinada organização quer estudar a proporção do PIB investido em educação nos países. Sabemos que a proporção será um número entre zero e um, pois se todo PIB for utilizado para investir na educação teríamos a proporção máxima, e se nada do PIB fosse investido em educação seria o caso oposto, ou seja, proporção mínima. Claro que dificilmente haverá casos extremos nesse exemplo. Portanto, é de suma importância que o modelo probabilístico tenha como suporte o intervalo unitário. Além disso, sabemos que provavelmente haverá uma diferença no investimento em educação nos países desenvolvidos e subdesenvolvidos caracterizando duas populações de países; então quanto mais flexível for o modelo probabilístico mais rica será a modelagem.

No contexto de dados com suporte limitado, especificamente no intervalo $(0,1)$, existem várias distribuições de probabilidade que podem ser consideradas. Uma das distribuições mais comuns e a mais utilizada é a distribuição beta (Gupta e Nadarajah, 2004). Na parametrização de Ferrari e Cribari-Neto (2004) os parâmetros da distribuição beta são interpretados como média e precisão. Entretando, a distribuição beta não tem função distribuição acumulada (fda) em forma fechada. Uma distribuição alternativa é a distribuição Kumaraswamy (Kumaraswamy, 1980), que tem fda e, consequentemente, quantis em forma explícita. Além das distribuições beta e Kumaraswamy, apresentaremos as distribuições simplex, gama unitária e beta retangular. Como alternativa a estas distribuições, Smithson e Merkle (2013) apresentam um mecanismo que permite gerar distribuições de probabilidade no intervalo unitário induzidas por transformações. Neste mecanismo é possível partir das distribuições mencionadas em Ley (2015) e Jones (2015) e gerar novas distribuições flexíveis com suporte 
no intervalo unitário.

Nesta dissertação apresentamos as principais distribuições contínuas definidas no intervalo unitário, além de duas classes de distribuições obtidas por transformações recentemente apresentadas na literatura estatística. Estudamos propriedades dessas distribuições e as utilizamos para aplicações a dados reais. Por fim, criamos uma página online para analisar dados observados no intervalo unitário, considerando todas as distribuições estudadas nesta dissertação.

\subsection{Organização da dissertação}

Esta dissertação é composta por cinco capítulos. No Capítulo 2 apresentamos as principais e usuais distribuições no intervalo unitário; apresentamos suas função densidade de probabilidade (fdp), fda e momentos. Além disso, ilustramos algumas formas que essas distribuições de probabilidade podem assumir. Para cada distribuição apresentamos sua parametrização original e uma outra parametrização que permite, quando possível, trazer interpretabilidade aos parâmetros. Exploramos essa interpretabilidade e discutimos quais são as relações dos parâmetros com as possíveis formas da distribuição.

No Capítulo 3 apresentamos uma nova classe de distribuições, que originalmente foi sugerida por Smithson e Merkle (2013, Seção 6.3). Como alternativa às distribuições usuais, esta classe apresenta algumas características particulares, que podem representar vantagens ou desvantagens. Essa classe de distribuição é bastante ampla, e nos concentraremos em duas possíveis subclasses. A primeira é a classe de distribuições generalized Johnson $S_{B}$ proposta por Lemonte e Bazán (2015), que aqui chamaremos de distribuição logito. A segunda, uma nova família de distribuições, chamaremos de logito skew.

No Capítulo 4 utilizamos as distribuições de probabilidade apresentadas nos Capítulos 2 e 3 para modelar dados de algumas variáveis do tipo proporção coletadas pelo Banco Mundial referentes aos países do mundo.

Para finalizar, no Capítulo 5 apresentamos nossas considerações finais, com sugestões de trabalhos futuros para o tema em questão. 


\section{Capítulo 2}

\section{Principais distribuições no intervalo unitário}

Seja $(a, b)$, com $-\infty<a<b<\infty$, um intervalo limitado na reta real. Seja $g:(a, b) \rightarrow \mathbb{R}^{+}$ uma função contínua tal que $\int_{a}^{b} g(y) d y<\infty$. Então, $f:(a, b) \rightarrow \mathbb{R}^{+}$definida como

$$
f(y)=\frac{g(y)}{\int_{a}^{b} g(y) d y}, \quad y \in(a, b),
$$

é uma função densidade de probabilidade (fdp) no intervalo $(a, b)$. Existem, portanto, infinitas distribuições em intervalos limitados, em particular, no intervalo unitário, $(0,1)$.

It is one of the easiest things in statistics to invent new univariate distributions; after all, any non-negative integrable function is the core of a density function. The ongoing challenge is to extract from the overwhelming plethora of possibilities those relatively few with the best and most appropriate properties that are of real potential value in practical applications. (Jones, 2015, p. 189)

As distribuições de probabilidade utilizadas para fins de modelagem estatística dependem de parâmetros que determinam suas possíveis formas. É desejável que as distribuições não tenham muitos parâmetros, sejam flexíveis no sentido de acomodar formas diversificadas, tenham parâmetros interpretáveis e sejam tratáveis para fins de inferência acerca de seus parâmetros.

Neste capítulo, apresentamos uma breve revisão sobre algumas distribuições de probabilidade no intervalo unitário, a saber: beta, Kumaraswamy, simplex, gama unitária e beta retangular. Apresentamos suas fdp, fda e momentos. Possíveis formas para as fdp de cada distribuição são exibidas em gráficos estátiscos e dinâmicos. Para acesso aos gráficos dinâmicos clique aqui ${ }^{1}$. Além disso, incluimos alguns comentários que particularizam cada distribuição. Uma exploração ampla e detalhada sobre distribuições de probabilidade com suporte limitado é encontrada em Kotz e Van Dorp (2004).

\section{Distribuição beta}

Dizemos que $Y$ tem distribuição beta com parâmetros de forma $\alpha>0$ e $\beta>0$, e escrevemos $Y \sim \operatorname{Beta}(\alpha, \beta)$, se sua fdp é dada por

$$
f_{0}(y ; \alpha, \beta)=\frac{\Gamma(\alpha+\beta)}{\Gamma(\alpha) \Gamma(\beta)} y^{\alpha-1}(1-y)^{\beta-1}, \quad y \in(0,1)
$$

\footnotetext{
${ }^{1}$ https://francimario.shinyapps.io/unit_data/\#section-models
} 
em que $\Gamma(\cdot)$ é a função gama, isto é, $\Gamma(t)=\int_{0}^{\infty} x^{t-1} e^{-x} d x$. A fda de $Y$ não tem forma fechada. A média e a variância de $Y$ são dadas, respectivamente, por

$$
\mathrm{E}(Y)=\frac{\alpha}{\alpha+\beta}, \quad \operatorname{Var}(Y)=\frac{\alpha \beta}{(\alpha+\beta)^{2}(\alpha+\beta+1)} .
$$

Ferrari e Cribari-Neto (2004) propõem uma parametrização alternativa para a distribuição beta. Seja $\mu=\mathrm{E}(Y)$ e $\phi=\alpha+\beta$. Dessa forma, $\operatorname{Var}(Y)=\mu(1-\mu) /(1+\phi)$ e $\phi$ pode ser interpretado como um parâmetro de precisão. Dizemos que $Y$ tem distribuição beta de média $\mu \in(0,1)$ e parâmetro de precisão $\phi>0$ se sua fdp é dada por

$$
f(y ; \mu, \phi)=\frac{\Gamma(\phi)}{\Gamma(\mu \phi) \Gamma((1-\mu) \phi)} y^{\mu \phi-1}(1-y)^{(1-\mu) \phi-1}, \quad y \in(0,1),
$$

e escrevemos $Y \sim \operatorname{beta}(\mu, \phi)$.

A distribuição beta tem a propriedade da reflexão, isto é, se $Y \sim \operatorname{beta}(\mu, \phi)$, então $1-Y \sim \operatorname{beta}(1-\mu, \phi)$. Se $\mu=0.5$ e $\phi=2, Y$ tem distribuição uniforme no intervalo $(0,1)$.

A Figura 2.1 mostra gráficos de fdp da distribuição beta $(\mu, \phi)$ para várias configurações de parâmetros. Nota-se que as densidades podem assumir diferentes formas, a saber: unimodal, simétrica, assimétrica, U, J, J invertido, til e uniforme. Para assumir forma unimodal basta $\mu \phi>1 \mathrm{e}(1-\mu) \phi>1$. Para assumir forma simétrica $\mu=0.5$ e, consequentemente, assimétrica $\mu \neq 0.5$. Para forma de U deve-se ter $\mu \phi<1$ e $(1-\mu) \phi<1$. Para assumir forma de J é necessário que $\mu \phi>1$ e $(1-\mu) \phi=1$. Para assumir forma de J invertido é necessário que $(1-\mu) \phi>1$ e $\mu \phi=1$. Para assumir forma de til é necessário que $\mu \phi>1$ e $(1-\mu) \phi<1$. Para assumir forma de til invertido é necessário que $(1-\mu) \phi>1$ e $\mu \phi<1$.

\section{Distribuição Kumaraswamy}

Dizemos que $Y$ tem distribuição Kumaraswamy com parâmetros de forma $\alpha>0$ e $\beta>0$, e escrevemos $Y \sim \operatorname{Kumaraswamy}(\alpha, \beta)$, se sua fdp é dada por

$$
f_{0}(y ; \alpha, \beta)=\alpha \beta y^{\alpha-1}\left(1-y^{\alpha}\right)^{\beta-1}, \quad y \in(0,1) .
$$

Essa distribuição, proposta por Kumaraswamy (1980), tem fda em forma fechada dada por

$$
F_{0}(y ; \alpha, \beta)=1-\left(1-y^{\alpha}\right)^{\beta}, \quad y \in(0,1) .
$$

Consequentemente, o quantil de ordem $p$ também tem forma fechada, e é dado por

$$
y_{p}=\left[1-(1-p)^{1 / \beta}\right]^{1 / \alpha}, \quad p \in(0,1) .
$$

O momento de ordem $n$ de $Y$ é dado por

$$
\mathrm{E}\left(Y^{n}\right)=\beta \mathrm{B}(1+n / \alpha ; \beta)
$$

em que $\mathrm{B}(\cdot, \cdot)$ é a função beta, $\mathrm{B}(x, y)=\int_{0}^{1} t^{x-1}(1-t)^{y-1} d t$. 


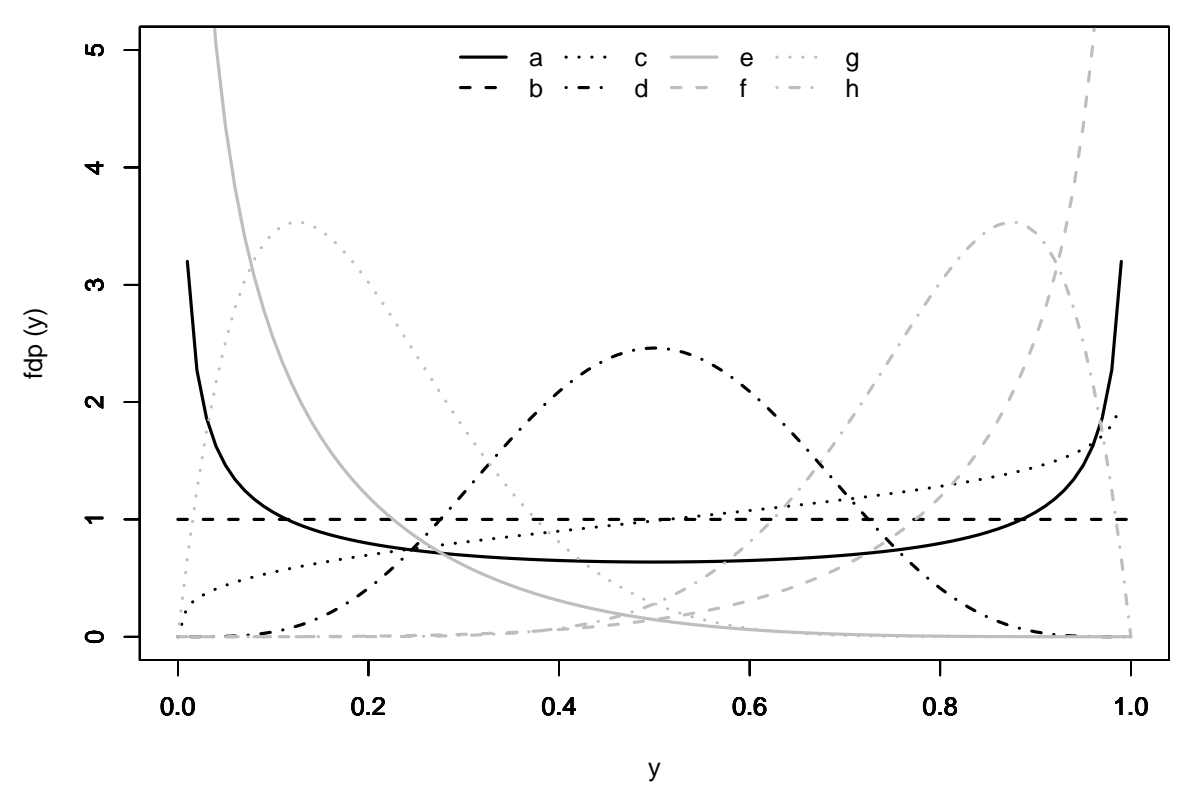

Figura 2.1: Funçôes densidade de probabilidade da distribuição beta $(\mu, \phi):(a)(\mu, \phi)=(0.5,1)$, (b) $(\mu, \phi)=(0.5,2)$, (c) $(\mu, \phi)=(0.6,2.2)$, (d) $(\mu, \phi)=(0.5,10)$, (e) $(\mu, \phi)=(0.1,5)$, (f) $(\mu, \phi)=$ $(0.9,5),(g)(\mu, \phi)=(0.2,10)$ e $(h)(\mu, \phi)=(0.8,10)$

Uma parametrização alternativa foi proposta por Mitnik e Baek (2013) em termos de um vetor de parâmetros $(\mu, \phi)$, em que

$$
\mu=\left[1-\exp \left(\frac{\ln (0.5)}{\beta}\right)\right]^{1 / \alpha}
$$

é a mediana de $Y$ e

$$
\phi=\alpha
$$

é visto como um parâmetro de precisão.

Assim, dizemos que $Y$ tem distribuição de Kumaraswamy com mediana $\mu \in(0,1)$ e parâmetro de precisão $\phi>0$ se a fdp e fda são

$$
f(y ; \mu, \phi)=\frac{\phi \log (0.5)}{\log \left(1-\mu^{\phi}\right)} y^{\phi-1}\left(1-y^{\phi}\right)^{\frac{\log (0.5)}{\log \left(1-\mu^{\phi}\right)}-1}, \quad y \in(0,1),
$$

e

$$
F(y ; \mu, \phi)=1-\left(1-y^{\phi}\right)^{\frac{\log (0.5)}{\log \left(1-\mu^{\phi}\right)}}, \quad y \in(0,1),
$$

respectivamente, e escrevemos $Y \sim \operatorname{Kuma}(\mu, \phi)$. O quantil de ordem $p$ fica dado por

$$
y_{p}=\left[1-(1-p)^{\frac{\log \left(1-\mu^{\phi}\right)}{\log (0.5)}}\right]^{1 / \phi}, \quad p \in(0,1)
$$

Sejam $X$ e $Y$ duas variáveis aleatórias (v.a.'s) arbitrárias com fda $F$ e $G$, respectivamente. Dize-se que $X$ é mais dispersa do que $Y$ se $F^{-1}(v)-F^{-1}(u) \geq G^{-1}(v)-G^{-1}(u)$ para 
todo $0<u<v<1$. Consequentemente, definindo $Q S_{X}(u)=F^{-1}(1-u)-F^{-1}(u)$ e $Q S_{Y}(u)=G^{-1}(1-u)-G^{-1}(u)$ para $u \in(0,0.5)$, temos que $X$ é mais dispersa do que $Y$ se $Q S_{X}(u) \geq Q S_{Y}(u)$. De fato, $\phi$ é um parâmetro de precisão pois Mitnik e Baek (2013) mostram que, se $X \sim \operatorname{Kuma}\left(\mu, \phi_{x}\right)$ e $Y \sim \operatorname{Kuma}\left(\mu, \phi_{y}\right) \operatorname{com} \phi_{y} \leq \phi_{x}$, então $Q S_{X}(u) \leq$ $Q S_{Y}(u)$ para $\mu \in(0,0.5)$.

Um resultado interessante utilizando a parametrização padrão desta distribuição é que, se $Y=X^{n}, n>0$, então $X \sim \operatorname{Kumaraswamy}(\alpha, \beta)$ se e somente se $Y \sim \operatorname{Kumaraswamy}(\alpha / n, \beta)$. Quando $n=\alpha, Y \sim \operatorname{Beta}(1, \beta)$.

Na Figura 2.2 estão expressos os gráficos da fdp da distribuição $\operatorname{Kuma}(\mu, \phi)$ para alguns pares de parâmetros. Assim como a distribuição beta a distribuição Kumaraswamy pode assumir as formas unimodal, U, J, J invertido e forma de til. Como 1-Y não tem distribuição Kumaraswamy então essa família de distribuições não tem a propriedade da reflexão, ou seja, $\exists t \in(0,1)$ tal que $f_{Y}(t) \neq f_{1-Y}(1-t)$. Uma característica desta distribuição é que se $\mu=0.5$ a distribuição não é simétrica, exceto quando $\phi=1$ que é o caso da distribuição uniforme. Para forma de U deve-se ter $\phi<1$ e $\mu=0.5$.

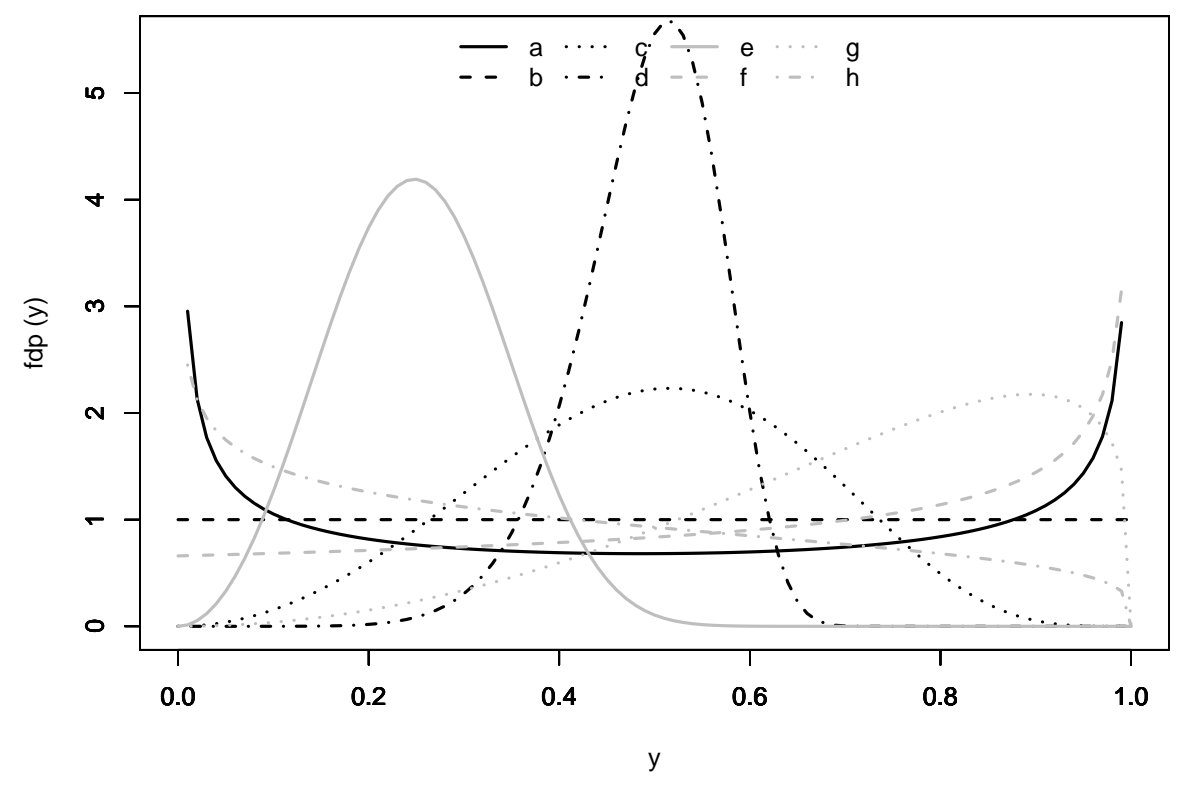

Figura 2.2: Funçôes densidade de probabilidade da distribuição Kuma $(\mu, \phi):(a)(\mu, \phi)=(0.5,0.5)$, (b) $(\mu, \phi)=(0.5,1)$, (c) $(\mu, \phi)=(0.5,3)$, (d) $(\mu, \phi)=(0.5,8)$, (e) $(\mu, \phi)=(0.25,3)$, (f) $(\mu, \phi)=$ $(0.65,1),(g)(\mu, \phi)=(0.75,3)$ e $(h)(\mu, \phi)=(0.35,8)$.

\section{Distribuição simplex}

Dizemos que $Y$ tem distribuição simplex com média $\mu \in(0,1)$ e parâmetro de precisão $\phi>0$, e denotamos $Y \sim \mathrm{S}(\mu, \phi)$, se sua fdp é dada por

$$
f(y ; \mu, \phi)=\left\{\frac{\phi}{2 \pi\{y(1-y)\}^{3}}\right\}^{\frac{1}{2}} \exp \left\{-\frac{\phi}{2} \frac{(y-\mu)^{2}}{y(1-y) \mu^{2}(1-\mu)^{2}}\right\}, \quad y \in(0,1) .
$$


Esta distribuição foi proposta por Barndorff-Nielsen e Jørgensen (1991) e sua fda não tem forma fechada. Sua média e variância são

$$
\mathrm{E}(Y)=\mu, \quad \operatorname{Var}(Y)=\mu(1-\mu)-\sqrt{\frac{\phi}{2}} \exp \left\{\frac{\phi}{2 \mu^{2}(1-\mu)^{2}}\right\} \Gamma\left\{\frac{1}{2} ; \frac{\phi}{2 \mu^{2}(1-\mu)^{2}}\right\},
$$

em que $\Gamma(t ; x)=\int_{x}^{\infty} y^{t-1} e^{-y} d y$ é a função gama incompleta.

Assim como a distribuição beta, a distribuição simplex tem a propriedade de reflexão, ou seja, se $Y \sim \mathrm{S}(\mu, \phi)$, então $1-Y \sim \mathrm{S}(1-\mu, \phi)$.

A Figura 2.3 apresenta gráficos da fdp da distribuição $\mathrm{S}(\mu, \phi)$ para diferentes configurações de parâmetros $\mu$ e $\phi$. Essa distribuição pode apresentar as formas simétrica unimodal ou bimodal e assimétrica. Nesta distribuição não é possível obter a distribuição uniforme. Para assumir forma simétrica é necessário que $\mu=0.5$ e para assumir forma assimétrica é necessário que $\mu \neq 0.5$.

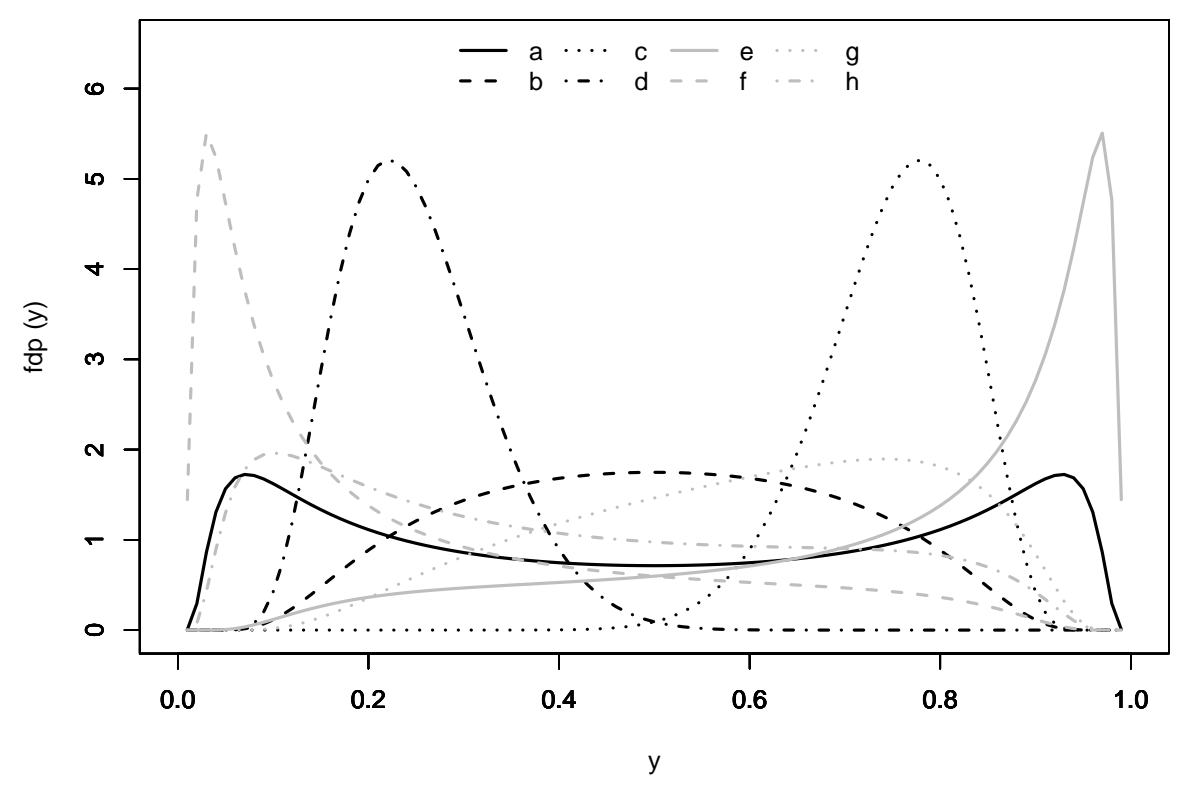

Figura 2.3: Funçôes densidade de probabilidade da distribuição $S(\mu, \phi):(a)(\mu, \phi)=(0.5, .05)$, (b) $(\mu, \phi)=(0.5,0.3)$, (c) $(\mu, \phi)=(0.75,1)$, (d) $(\mu, \phi)=(0.25,1),(e)(\mu, \phi)=(0.75,0.05),(f)$ $(\mu, \phi)=(0.25,0.05),(g)(\mu, \phi)=(0.6,0.25)$ e $(h)(\mu, \phi)=(0.4,0.1)$.

\section{Distribuição gama unitária}

Dizemos que $Y$ tem distribuição gama unitária com parâmetros $\alpha>0$ e $\tau>0$, e escrevemos $Y \sim \operatorname{GU}(\alpha, \tau)$, se sua fdp é dada por

$$
f_{0}(y ; \alpha, \tau)=\frac{\alpha^{\tau}}{\Gamma(\tau)} y^{\alpha-1}\left[\log \left(\frac{1}{y}\right)\right]^{\tau-1}, \quad y \in(0,1) .
$$

A família de distribuições gama unitária foi apresentada por Grassia (1977) e o motivo 
desse nome é pelo fato de que se $X$ segue uma distribuição gama com parâmetros de forma $\alpha>0$ e $\tau>0$, então $Y=e^{-X}$ segue uma distribuição gama unitária com parâmetros de forma $\alpha>0$ e $\tau>0$. A fda não tem expressão fechada. A média e a variância de $Y$ são

$$
\mathrm{E}(Y)=\left[\frac{\alpha}{\alpha+1}\right]^{\tau}, \quad \operatorname{Var}(\mathrm{Y})=\left\{\left[\frac{\alpha}{\alpha+2}\right]^{\tau}-\left[\frac{\alpha}{\alpha+1}\right]^{2 \tau}\right\}=\mu\left[\frac{1}{\left(2-\mu^{\frac{1}{\tau}}\right)^{\tau}}-\mu\right]
$$

respectivamente. A variância depende da média, para $\mu$ fixado, e diminui quando $\tau$ cresce. Logo, $\tau$ pode ser interpretado como um parâmetro de precisão. Além disso, quando $\tau \rightarrow \infty$ temos que a variância converge a $\mu(1-\mu)$, que coincide com a variância da distribuição $\operatorname{beta}(\mu, \phi)$ quando $\phi \rightarrow 0$.

Sejam $\mu=\mathrm{E}(Y)$ e $\phi=\tau$. Dizemos que $Y$ tem distribuição gama unitária com média $\mu$ e parâmetro de precisão $\phi$ se sua fdp é dada por

$$
f(y ; \mu, \phi)=\frac{\left[\frac{\mu^{1 / \phi}}{1-\mu^{1 / \phi}}\right]^{\phi}}{\Gamma(\phi)} y^{\frac{\mu^{1 / \phi}}{1-\mu^{1 / \phi}}-1}\left[\log \left(\frac{1}{y}\right)\right]^{\phi-1}, \quad y \in(0,1)
$$

e escrevemos $Y \sim \operatorname{Gu}(\mu, \phi)$.

A Figura 2.4 exibe gráficos da fdp da distribuição $\mathrm{Gu}(\mu, \phi)$ para alguns pares de parâmetros $\mu$ e $\phi$. Se $\mu=0.5$ e $\phi=1$ temos a distribuição uniforme; além disso, é possível obter as formas assimétrica, U, J, J invertido e til. Assim como a distribuição Kumaraswamy a distribuição gama unitária não tem a propriedade da reflexão. O único caso de perfeita simetria é quando $\mu=0.5$ e $\phi=1$ e corresponde à distribuição uniforme. Para forma de U deve-se ter $\phi<1$ e $\mu=0.5$. Para as formas J e J invertido é necessário $\mu$ ser próximo de 1 e 0 , respectivamente e $\phi=1$, novamente característica similar a distribuição Kuma.

\section{Distribuição beta retangular}

Dizemos que $Y$ tem distribuição beta retangular com parâmetros $\mu \in(0,1), \phi>0$ e $\theta \in[0,1]$, e escrevemos $Y \sim \operatorname{BRr}(\mu, \phi, \theta)$, se sua fdp é dada por

$$
f(y ; \mu, \phi, \theta)=\theta+(1-\theta) \mathrm{b}(y ; \mu, \phi), \quad y \in(0,1),
$$

em que $\mathrm{b}(y ; \mu, \phi)$ é a fdp da distribuição beta $(\mu, \phi)$.

A família de distribuições beta retangular foi proposta por Hahn (2008) e é uma generalização da família de distribuições beta. Surgiu com a necessidade de considerar áreas de cauda e uma maior flexibilidade com respeito à variância segundo Hahn (2008) e García et al. (2011). Note que se $\theta=1$ teremos uma distribuição uniforme e se $\theta=0$, uma distribuição beta. Como a distribuição beta não tem fda com expressão fechada, a distribuição beta retangular também não tem, exceto para $\theta=1$. A distribuição beta retangular é uma mistura de uma distribuição uniforme, ou seja, beta $(0.5,2)$, e uma distribuição beta $(\mu, \phi)$. Sendo assim, temos que a média e variância são

$$
\mathrm{E}(Y)=\frac{\theta}{2}+(1-\theta) \mu, \quad \operatorname{Var}(Y)=\frac{\mu(1-\mu)}{1+\phi}(1-\theta)(1+\theta(1+\phi))+\frac{\theta}{12}(4-3 \theta),
$$




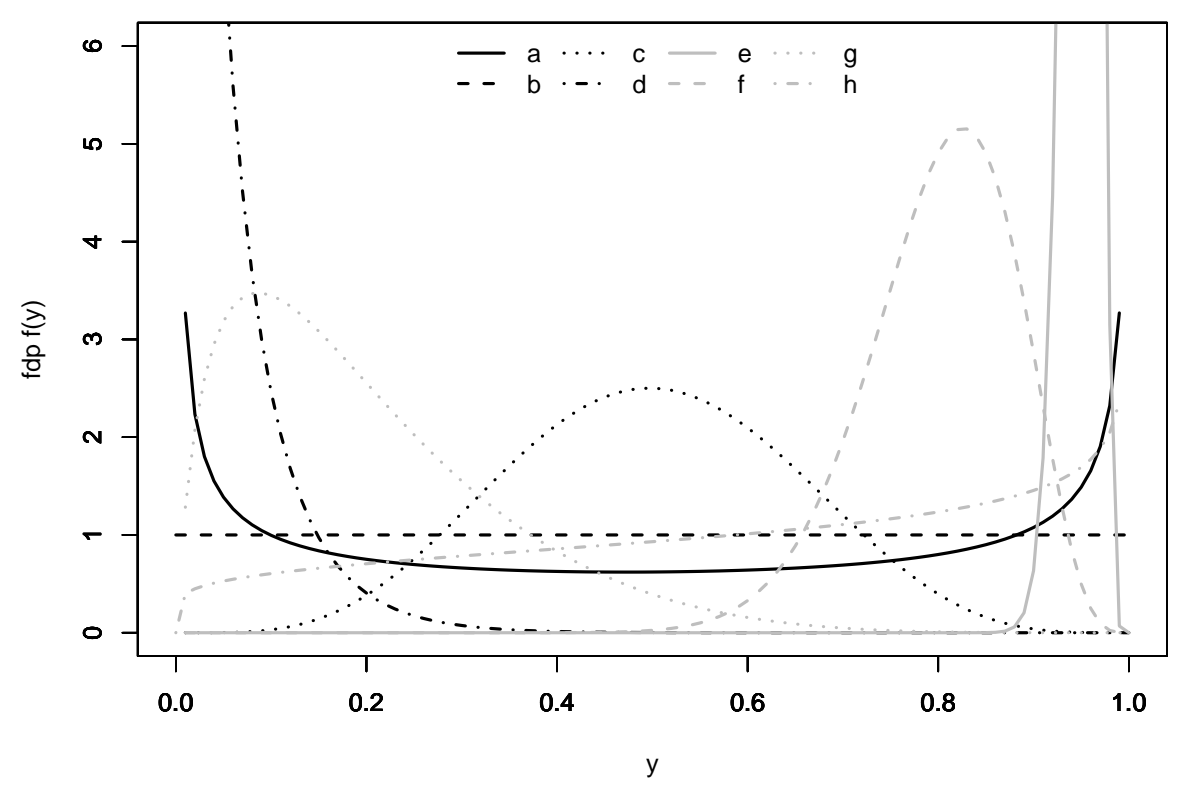

Figura 2.4: Funçôes densidade de probabilidade da distribuição $G u(\mu, \phi):(a)(\mu, \phi)=(0.5,0.5)$, (b) $(\mu, \phi)=(0.5,1)$, (c) $(\mu, \phi)=(0.5,5)$, (d) $(\mu, \phi)=(0.05,9)$, (e) $(\mu, \phi)=(0.95,9)$, (f) $(\mu, \phi)=$ $(0.8,5),(g)(\mu, \phi)=(0.2,5)$ e $(h)(\mu, \phi)=(0.6,0.9)$.

respectivamente.

Uma nova parametrização apresentada por Bayes et al. (2012) considera

$$
\gamma=\mathrm{E}(Y)=\frac{\theta}{2}+(1-\theta) \mu, \quad \alpha=\frac{\theta}{1-(1-\theta)|2 \mu-1|} .
$$

Dessa forma, o espaço paramétrico de $\theta$ tem uma restrição, isto é, $0<\theta<1-|2 \gamma-1|$. Neste caso, $(\gamma, \alpha) \in[0,1]^{2}$ e $\phi>0$. Temos que $Y$ tem distribuição beta retangular com média $\gamma$, parâmetro de precisão $\phi$ e parâmetro de forma associado às caudas $\alpha$ se sua fdp é dada por $f(y ; \gamma, \phi, \alpha)=\alpha(1-|2 \gamma-1|)+(1-\alpha(1-|2 \gamma-1|)) \mathrm{b}\left(y ; \frac{\gamma-0.5 \alpha(1-|2 \gamma-1|)}{1-\alpha(1-|2 \gamma-1|)}, \phi\right), \quad y \in(0,1)$, em que $\mathrm{b}(y ; \mu, \phi)$ é a fdp da distribuição beta $(\mu, \phi)$, e escrevemos $Y \sim \operatorname{BR}(\gamma, \phi, \alpha)$.

A Figura 2.5 exibe gráficos da fdp da distribuição beta retangular para diferentes valores de $\gamma, \phi$ e $\alpha$. Além das formas possíveis da distribuição beta ainda é possível considerar formas em que $\lim _{y \rightarrow 0} f(y) \neq 0(<+\infty)$ e $\lim _{y \rightarrow 1} f(y) \neq 0(<+\infty)$ quando $\alpha>0$.

Neste capítulo apresentamos as principais distribuições de probabilidade cujo suporte é o intervalo $(0,1)$. Notamos que todas distribuições conseguem assumir, algumas com limitação, formas simétricas e assimétricas, algumas também conseguem formas J e J invertido de til. Há certas características de cada distribuição que vale a pena ressaltar. Não existe uma distribuição simplex que seja uniforme. Pode ser bimodal, mas a posição das modas não é flexível, sendo assim, uma alternativa seria utilizar misturas de distribuições para obter multimodalidade. Dentre essas distribuições a distribuição beta retangular é a única que consegue ter caudas mais pesadas. Entretanto, mudanças no parâmetro de cauda $(\alpha)$ 


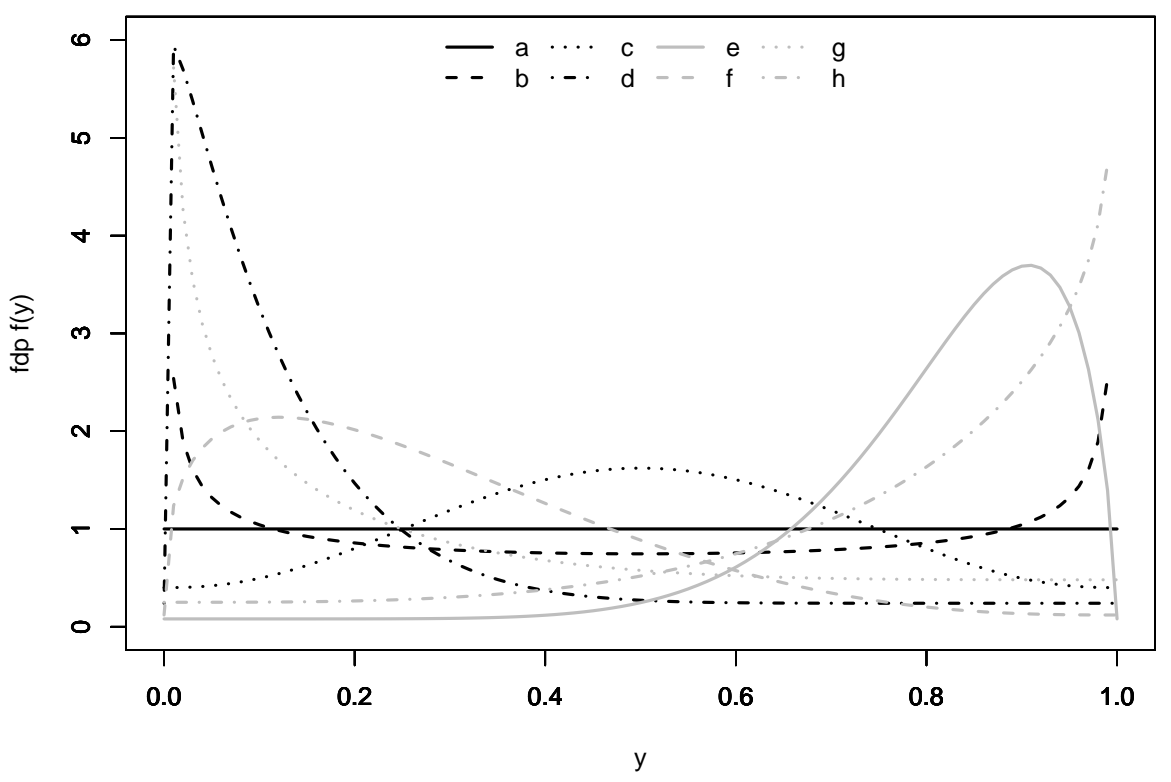

Figura 2.5: Funçôes densidade de probabilidade da distribuição $B R(\gamma, \phi, \alpha):(a)(\gamma, \phi, \alpha)=$ $(0.5,2,0.7)$, (b) $(\gamma, \phi, \alpha)=(0.5,1,0.3)$, (c) $(\gamma, \phi, \alpha)=(0.5,7,0.4),(d)(\gamma, \phi, \alpha)=(0.2,10,0.6)$, (e) $(\gamma, \phi, \alpha)=(0.8,10,0.2)$, (f) $(\gamma, \phi, \alpha)=(0.3,5,0.2)$, (g) $(\gamma, \phi, \alpha)=(0.3,5,0.8)$ e (h) $(\gamma, \phi, \alpha)=$ $(0.75,5,0.5)$.

altera simultaneamente ambas as caudas. Pelo nosso breve estudo podemos perceber que as distribuições beta, Kumaraswamy e gama unitária apresentam formas relativamente bem similares.

Com essas distribuições já é possível modelar bases de dados para as mais diversas formas no intervalo $(0,1)$. Além disso, pode-se estimar de maneira relativamente simples os parâmetros pelo método da máxima verossimilhança. Comumente o interesse não é apenas estimar os parâmetros, mas também um quantil da distribuição. Portanto, pode ser desejável que a função de distribuição acumulada tenha forma fechada. Neste caso pela propriedade de invariância dos estimadores de máxima verossimilhança facilmente consegue-se estimar quantis. Das distribuições apresentadas apenas a distribuição Kumaraswamy tem forma fechada para a função de distribuição acumulada. Na Tabela 2.1 apresentamos um resumo de algumas características das distribuições apresentadas neste capítulo.

Tabela 2.1: Algumas características das principais distribuições.

\begin{tabular}{l|ccccc}
\hline & Beta & Kuma & Simplex & Gu & BR \\
\hline Simétrica & $\operatorname{sim}$ & não & $\operatorname{sim}$ & $\operatorname{sim}$ & $\operatorname{sim}$ \\
Assimétrica & $\operatorname{sim}$ & $\operatorname{sim}$ & $\operatorname{sim}$ & $\operatorname{sim}$ & $\operatorname{sim}$ \\
J e J invertido & $\operatorname{sim}$ & $\operatorname{sim}$ & não & $\operatorname{sim}$ & $\operatorname{sim}$ \\
U & $\operatorname{sim}$ & $\operatorname{sim}$ & não & $\operatorname{sim}$ & $\operatorname{sim}$ \\
Bimodal & não & não & sim & não & não \\
Til e til invertido & $\operatorname{sim}$ & $\operatorname{sim}$ & não & sim & sim \\
Reflexão & $\operatorname{sim}$ & não & sim & não & sim \\
Fda fechada & não & sim & não & não & não \\
\hline
\end{tabular}




\section{Capítulo 3}

\section{Distribuições no intervalo unitário geradas por transformações}

\subsection{Introdução}

Smithson e Merkle (2013, Seção 6.3) apresentam uma família de distribuições relativamente pouco explorada. Seja $H:(-\infty, \infty) \rightarrow(0,1)$ uma transformação inversível. Defina $Y=H(X)$, em que $X$ é uma v.a. definida no domínio de $H$ com fdp $g(x)$. Então a fdp da v.a. $Y$ é

$$
f(y)=g\left(H^{-1}(y)\right) \frac{\partial H^{-1}(y)}{\partial y} .
$$

A princípio, $X$ pode ter qualquer distribuição na reta real, porém os autores sugerem que $X$ seja simétrica padrão.

Uma v.a. $W$ segue uma distribuição simétrica se sua fdp é dada por $h(w)=\sqrt{\phi} g(\phi(w-$ $\left.\mu)^{2}\right), w \in \mathbb{R}, \mu \in \mathbb{R}$ e $\phi>0$. A função $g: \mathbb{R} \rightarrow[0, \infty)$ é tal que $\int_{0}^{\infty} u^{-1 / 2} g(u) d u=1$. Escrevemos $W \sim \mathrm{S}(\mu, \phi ; g)$. A função $g$, que é independente de $\mu$ e $\phi$, é conhecida como função geradora de densidades. Para mais detalhes sobre distribuições simétricas ver Anderson e Fang (1990). Sempre que existem, $\mathrm{E}(W)=\mu$ e $\operatorname{Var}(W)=\xi \phi^{-1}$, em que $\xi$ não depende de parâmetros. Se $W \sim \mathrm{S}(0,1 ; g)$ dizemos que $W$ tem distribuição simétrica padrão.

Como mencionado anteriormente, $X$ pode ter qualquer distribuição na reta real. Sendo assim, uma outra classe de distribuições que pode ser utilizada para gerar distribuições no intervalo unitário é a classe de distribuições assimétricas, ou simplesmente skew, que foi apresentada inicialmente por Azzalini (1985) e outros autores para o caso normal. Posteriormente, Gupta et al. (2002) estenderam a ideia para as distribuições uniforme, t, Cauchy, Laplace e logística, apresentando os quatro primeiros momentos.

Sejam $W_{0} \sim \mathrm{S}\left(0,1 ; g_{0}\right)$ com fdp $g_{W_{0}}(w)$ e $W_{1} \sim \mathrm{S}\left(0,1 ; g_{1}\right)$ com fda $G_{W_{1}}(w)$. Gupta et al. (2002) consideram a função

$$
g_{W}(w \mid \lambda)=2 g_{W_{0}}(w) G_{W_{1}}(\lambda w), \quad \lambda \in \mathbb{R},
$$

e mostra que é uma fdp. Não é necessário que $G_{W_{1}}^{\prime}(w)=g_{W_{0}}(w)$, mas aqui será, e neste caso

$$
g_{W}(w \mid \lambda)=2 g_{W_{0}}(w) G_{W_{0}}(\lambda w), \quad \lambda \in \mathbb{R},
$$

em que $G_{W_{0}}^{\prime}(\lambda w)=g_{W_{0}}(w)$. O parâmetro $\lambda$ controla a assimetria de $g_{W}$ : se $\lambda>0(\lambda<0)$, 
$g_{W}$ é assimétrica à direita (à esquerda) e se $\lambda=0, g_{W}$ é simétrica em torno de zero. Dizemos que $W$ tem distribuição skew padrão com parâmetro de assimetria $\lambda$ e função geradora de densidade $g$ se sua fdp é dada por (3.1) e escrevemos $W \sim \operatorname{Skew}(0,1, \lambda ; g)$. Esta distribuição pode ser estendida por localização e escala tomando a transformação $W^{\prime}=\mu+X / \sqrt{\phi}, \mu \in$ $\mathbb{R}, \phi>0$, mas esta extensão não será necessária no que segue.

Daqui em diante, tomamos

$$
Y=H_{\mu, \phi}(X)=H(\mu+X / \sqrt{\phi}),
$$

em que $\mu \in \mathbb{R}, \phi>0$ e $X \sim \mathrm{S}(0,1 ; g)$ ou $X \sim \operatorname{Skew}(0,1, \lambda ; g)$.

A família de distribuições de $Y$ tem como casos particulares a distribuição Johnson $\mathrm{S}_{\mathrm{b}}$, que foi apresentada por Johnson (1949), e a distribuição logito logística (LL), que foi apresentada por Tadikamalla e Johnson (1982). Se a função $H$ e a fda da v.a. $X$ têm forma fechada, então a distribuição de $Y$ tem fda em forma fechada, como é o caso da distribuição LL, para a qual $H$ é a função logística e a v.a. $X$ segue uma distribuição logística. Assim, a distribuição LL é uma classe muito útil para modelar quantis.

\subsection{Transformação logito}

Utilizando a transformação logística $H(x)=1 /\left(1+e^{-x}\right)$ temos, de (3.2), que

$$
Y=\frac{1}{1+e^{-(\mu+X / \sqrt{\phi})}}
$$

em que $X \sim \mathrm{S}(0,1 ; g)$ ou $X \sim \operatorname{Skew}(0,1, \lambda ; g)$.

Se $X \sim \mathrm{S}(0,1 ; g)$, então dizemos que $Y$ tem distribuição logito com parâmetros de localização $\mu$, dispersão $\phi$ e função geradora de densidades $g$ e escrevemos $Y \sim \operatorname{Logito}(\mu, \phi ; g)$. Denominação análoga é dada para o caso em que $X \sim \operatorname{Skew}(0,1, \lambda ; g)$ e escrevemos $Y \sim$ $\operatorname{LogitoS}(\mu, \phi, \lambda ; g)$.

Note que a transformação (3.3) é não-linear e, portanto, os momentos de $Y$ não são facilmente obtidos através dos momentos da distribuição de $X$. De fato, essa classe de distribuições não tem forma fechada para os momentos (Smithson e Merkle, 2013, p. 158). Uma alternativa é utilizar métodos numéricos para obtenção dos momentos.

Uma segunda alternativa é utilizar uma aproximação por meio da expansão em série de Taylor em torno de zero (série de Maclaurin), isto é, se $Y$ é dada por (3.3), então

$$
Y \approx \frac{1}{1+e^{-\mu}}+\frac{X e^{-\mu}}{\sqrt{\phi}\left(1+e^{-\mu}\right)^{2}}
$$

considerando apenas os dois primeiros termos da expansão. Dessa maneira, a média e a variância da distribuição de $Y$ são, aproximadamente,

$$
\mathrm{E}(Y) \approx \frac{\mu_{X} e^{-\mu}+\sqrt{\phi}\left(1+e^{-\mu}\right)}{\sqrt{\phi}\left(1+e^{-\mu}\right)^{2}}, \quad \operatorname{Var}(Y) \approx \xi \phi_{X}^{-1}\left[\frac{e^{-\mu}}{\phi\left(1+e^{-\mu}\right)^{2}}\right]^{2},
$$

em que $\mu_{X}=\mathrm{E}(X)$ e $\xi \phi_{X}^{-1}=\operatorname{Var}(X)$ caso existam. Se $\mu_{X}=0$ e $\xi \phi_{X}^{-1}=1$, então a média e 
a variância da distribuição de $Y$ são, aproximadamente,

$$
\mathrm{E}(Y) \approx \frac{1}{1+e^{-\mu}}, \quad \operatorname{Var}(Y) \approx\left[\frac{e^{-\mu}}{\phi\left(1+e^{-\mu}\right)^{2}}\right]^{2}
$$

A fdp e fda de $Y$ são

$$
f_{Y}(y ; \mu, \phi)=f_{X}\left(\sqrt{\phi}\left(y^{*}-\mu\right)\right) \frac{\sqrt{\phi}}{y(1-y)}, \quad y \in(0,1), \quad \phi>0, \quad \mu \in \mathbb{R},
$$

$\mathrm{e}$

$$
F_{Y}(y ; \mu, \phi)=F_{X}\left[\sqrt{\phi}\left(y^{*}-\mu\right)\right], \quad y \in(0,1), \quad \phi>0, \quad \mu \in \mathbb{R},
$$

em que $f_{X}(x)$ e $F_{X}(x)$ são, respectivamente, a fdp e fda de $X$ e $y^{*}=\log [y /(1-y)]$.

O quantil de ordem $p$ fica dado por

$$
y_{p}=\frac{1}{1+e^{-\left(F_{X}^{-1}(p) / \sqrt{\phi}+\mu\right)}} .
$$

Se a v.a. $X$ possui fda com expressão fechada, então $y_{p}$ também possui. Além disso, se $X$ for simétrica então a mediana da v.a. $Y$ fica dada por

$$
\operatorname{Med}(Y)=\frac{1}{1+e^{-\mu}}
$$

ou seja, $\mu=\log [\operatorname{Med}(Y) /(1-\operatorname{Med}(Y))]$.

O parâmetro $\phi$ é de precisão. De fato, considere, sem perda de generalidade, que $Y_{1} \sim$ $\operatorname{Logito}\left(0, \phi_{1}, g\right)$ e $Y_{2} \sim \operatorname{Logito}\left(0, \phi_{2}, g\right), \operatorname{com} \phi_{1}<\phi_{2}$. Para $p \in(0,0.5)$,

$$
\begin{gathered}
y_{2 p}-y_{1 p}>0 \Leftrightarrow \frac{1}{1+e^{-F_{X}^{-1}(p) / \sqrt{\phi_{2}}}}-\frac{1}{1+e^{-F_{X}^{-1}(p) / \sqrt{\phi_{1}}}}>0 \Leftrightarrow \\
e^{-F_{X}^{-1}(p) / \sqrt{\phi_{1}}}-e^{-F_{X}^{-1}(p) / \sqrt{\phi_{2}}}>0 \Leftrightarrow F_{X}^{-1}(p) / \sqrt{\phi_{2}}<F_{X}^{-1}(p) / \sqrt{\phi_{1}} \Leftrightarrow \phi_{1}<\phi_{2} .
\end{gathered}
$$

Por outro lado, para $p \in(0.5,1)$,

$$
y_{1 p}-y_{2 p}>0 \Leftrightarrow \phi_{1}<\phi_{2}
$$

Portanto, $\mathrm{QS}_{Y_{2}}<\mathrm{QS}_{Y_{1}}$.

Dois resultados importantes são apresentados a seguir.

(i) se $Y \sim \operatorname{Logito}(\mu, \phi$; g), então $1-Y \sim \operatorname{Logito}(-\mu, \phi$; g).

De fato, seja $Y \sim \operatorname{Logito}\left(\mu, \phi\right.$; g) e $Z=1-Y$, portanto $f_{Z}(z)=f_{Y}(1-z)$. De (3.6) temos que

$$
f_{Z}(z ; \mu, \phi)=f_{X}\left(\sqrt{\phi}\left(\log \frac{z}{1-z}+\mu\right)\right) \frac{\sqrt{\phi}}{z(1-z)}, \quad z \in(0,1), \quad \phi>0, \quad \mu \in \mathbb{R},
$$

que é a fdp de uma v.a. $Z \sim \operatorname{Logito}(-\mu, \phi ; \mathrm{g})$.

(ii) se $Y \sim \operatorname{LogitoS}(\mu, \phi, \lambda$; g), então $1-Y \sim \operatorname{LogitoS}(-\mu, \phi,-\lambda$; g). 
Seja $Y \sim \operatorname{LogitoS}(\mu, \phi, \lambda ; \mathrm{g})$ e $Z=1-Y$, portanto $f_{Z}(z)=f_{Y}(1-z)$. De (3.6) e (3.1) temos que

$$
\begin{aligned}
& f_{Z}(z ; \mu, \phi, \lambda)=g_{W_{0}}\left(\sqrt{\phi}\left(\log \frac{z}{1-z}+\mu\right)\right) G_{W_{0}}\left(-\lambda \sqrt{\phi}\left(\log \frac{z}{1-z}+\mu\right)\right) \frac{\sqrt{\phi}}{z(1-z)}, \quad z \in(0,1) \\
& \phi>0, \quad \mu \in \mathbb{R}, \text { que é a fdp de uma v.a. } Z \sim \operatorname{LogitoS}(-\mu, \phi,-\lambda ; \mathrm{g}) .
\end{aligned}
$$

Utilizando a transformação (3.3) e $X \sim \mathrm{N}(0,1) \operatorname{com} f(x)=e^{-x^{2} / 2} / \sqrt{2 \pi}$, temos a distribuição Johnson $\mathrm{S}_{\mathrm{B}}$ com fdp

$$
f_{Y}(y ; \mu, \phi)=\frac{\sqrt{\phi}}{\sqrt{2 \pi} y(1-y)} \exp \left\{-\frac{\phi}{2}\left(y^{*}-\mu\right)^{2}\right\}
$$

e fda

$$
F_{Y}(y ; \mu, \phi)=\Phi\left[\sqrt{\phi}\left(y^{*}-\mu\right)\right] .
$$

Nesta parametrização a distribuição Johnson $\mathrm{S}_{\mathrm{B}}$ é apresentada em Rennolls e Wang (2005) e, em parametrização alternativa, em Johnson (1949).

Utilizando a transformação (3.3) e $X \sim \mathrm{L}(0,1)$, ou seja, $X$ tem distribuição logística padrão com $f(x)=e^{-x} /\left(1+e^{-x}\right)^{2}$, temos a distribuição LL com fdp

$$
f_{Y}(y ; \mu, \phi)=\frac{(y /(1-y))^{-\sqrt{\phi}} e^{\mu \sqrt{\phi}}}{\left[1+(y /(1-y))^{-\sqrt{\phi}} e^{\mu \sqrt{\phi}}\right]^{2}} \frac{\sqrt{\phi}}{y(1-y)}
$$

e fda

$$
F_{Y}(y ; \mu, \phi)=\frac{1}{1+(y /(1-y))^{-\sqrt{\phi}} e^{\mu \sqrt{\phi}}} .
$$

Nesta parametrização esta distribuição é dada em Rennolls e Wang (2005) e, em outra parametrização, em Tadikamalla e Johnson (1982).

A parametrização utilizada aqui é a mesma do pacote gamıss (Rigby e Stasinopoulos, 2005; Stasinopoulos et al., 2017) que pode ser utilizado para fazer inferência.

Lemonte e Bazán (2015) estudaram essa classe de distribuições no caso em que a v.a. $X$ tem uma distribuição simétrica, apresentando algumas propriedades, métodos de estimação, estrutura de regressão e diagnóstico. Como ilustração utilizaram as distribuições normal, t generalizada, logística tipo I, logística generalizada, Kotz generalizada, exponencial dupla e potência estendida. Porém, a transformação utilizada pelos autores é

$$
Y=\frac{1}{1+e^{(X-\gamma) / \delta}}
$$

o que retorna uma parametrização diferente da que estamos trabalhando. Os parâmetros $\delta$ e $\lambda$ nessa classe são parâmetros de forma. Além disso, Lemonte e Bazán (2015) nomearam essa classe de generalized Johnson $S_{B}$ (GJS). Portanto, se $X \sim \mathrm{S}(0,1 ; g)$, em que $g$ é uma função geradora de densidade, então $Y \sim \operatorname{GJS}(\gamma, \delta ; g)$.

Nas duas próximas subseções apresentamos as distribuições logito obtidas através da transformação (3.3). Na Subseção 3.2.1 consideramos $X$ tendo uma distribuição simétrica, ou seja, a própria classe GJS e na Subseção 3.2.2 consideramos $X$ tendo uma distribuição assimétrica (skew). Por simplicidade, as distribuições aqui apresentadas serão logística, 
normal, t e exponencial potência. Para demais distribuições o mesmo raciocínio pode ser adotado. Enfatizamos que gráficos dinâmicos das fdp podem ser acessadas clicando aqui ${ }^{1}$.

\subsubsection{Classe logito}

Nesta subseção apresentamos as distribuições logito com $X$ tendo distribuição na classe simétrica, expressando as fdp's e fda's, assim como a média e variância utilizando a aproximação por expansão da série de Taylor, suas formas e alguns comentários que julgamos relevantes. As parametrizações que utilizaremos são as que estão implementas no pacote gamlss.

\section{Distribuição logito logística (LL)}

Se $X \sim \mathrm{L}(0,1)$, então dizemos que $Y$ dado em (3.3) tem distribuição logito logística com parâmetros $\mu \in \mathbb{R}$ e $\phi>0$, e escrevemos $Y \sim \operatorname{LL}(\mu, \phi)$, com fdp

$$
f(y ; \mu, \phi)=\frac{e^{-\sqrt{\phi}\left(y^{*}-\mu\right)}}{\left[1+e^{-\sqrt{\phi}\left(y^{*}-\mu\right)}\right]^{2}} \frac{\sqrt{\phi}}{y(1-y)}, \quad y \in(0,1),
$$

e fda

$$
F(y ; \mu, \phi)=\frac{1}{1+e^{-\sqrt{\phi}\left(y^{*}-\mu\right)}}, \quad y \in(0,1) .
$$

Como a fda tem forma fechada, então o quantil de ordem $p$ também tem e é dado por

$$
y_{p}=\frac{1}{1+e^{-\left(\phi^{-1 / 2} p^{*}+\mu\right)}},
$$

em que $p^{*}=\log [p /(1-p)]$.

A média e a variância da distribuição $\mathrm{L}(0,1)$ são $\mu_{X}=0$ e $\xi \phi_{X}^{-1}=\pi^{2} / 3$, respectivamente. Utilizando a aproximação dada em (3.4) temos que

$$
\mathrm{E}(Y) \approx \frac{1}{1+e^{-\mu}}, \quad \operatorname{Var}(Y) \approx \frac{\pi^{2} e^{-2 \mu}}{3 \phi^{2}\left(1+e^{-\mu}\right)^{4}}
$$

A Figura 3.1 mostra gráficos de fdp da distribuição $\operatorname{LL}(\mu, \phi)$ com várias configurações de $\mu$ e $\phi$. A distribuição LL pode assumir as formas simétrica, assimétrica, U, J e J invertido. Para a forma simétrica é necessário que $\mu=0$. Para forma assimétrica basta $\mu \neq 0$. Para forma de $\mathrm{U}, \mu=0$ e $\phi<1$. Para forma de $\mathrm{J}$ ou J invertido $\mu>0$ e $\mu<0$, respectivamente, e $\phi=1$. Como caso particular se $\mu=0$ e $\phi=1$ teremos a distribuição uniforme.

\section{Distribuição logito normal (LN)}

Se $X \sim \mathrm{N}(0,1)$, então dizemos que $Y$ dado em (3.3) tem distribuição logito normal com parâmetros $\mu \in \mathbb{R}$ e $\phi>0$, e escrevemos $Y \sim \operatorname{LN}(\mu, \phi)$, com fdp

$$
f(y ; \mu, \phi)=\frac{\sqrt{\phi}}{\sqrt{2 \pi} y(1-y)} \exp \left\{-\frac{\phi}{2}\left(y^{*}-\mu\right)^{2}\right\}, \quad y \in(0,1),
$$

\footnotetext{
${ }^{1}$ https://francimario.shinyapps.io/unit_data/\#section-models
} 


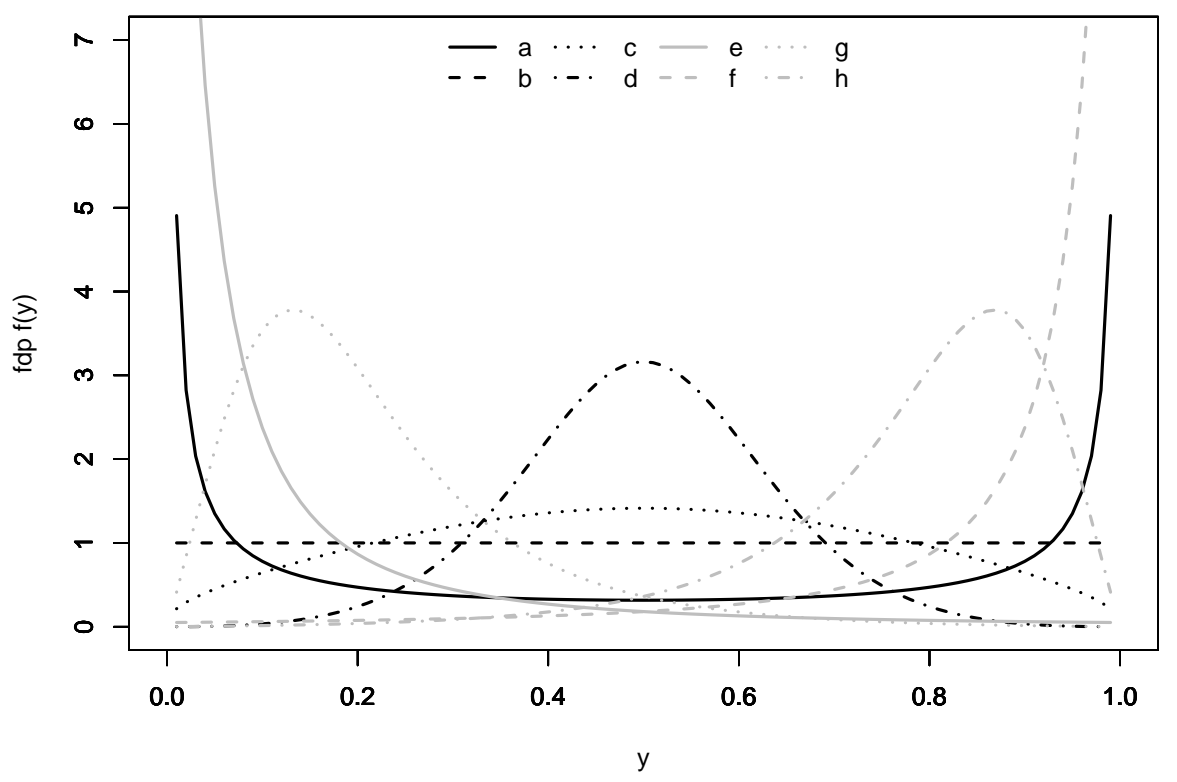

Figura 3.1: Funçôes densidade de probabilidade da distribuição $L L(\mu, \phi):(a)(\mu, \phi)=(0,0.1)$, (b) $(\mu, \phi)=(0,1)$, (c) $(\mu, \phi)=(0,2),(d)(\mu, \phi)=(0,10)$, (e) $(\mu, \phi)=(-3,1),(f)(\mu, \phi)=(3,1),(g)$ $(\mu, \phi)=(-1.5,4)$ e $(h)(\mu, \phi)=(1.5,4)$.

e fda

$$
F(y ; \mu, \phi)=\Phi\left[\sqrt{\phi}\left(y^{*}-\mu\right)\right], \quad y \in(0,1) .
$$

Como a fda não tem forma fechada então o quantil de ordem $p$ também não tem, mas fica dado por

$$
y_{p}=\frac{1}{1+e^{-\left(\phi^{-1 / 2} \Phi^{-1}(p)+\mu\right)}},
$$

em que $\Phi^{-1}(p)$ é o quantil de ordem $p$ da distribuição $N(0,1)$.

A média e a variância da distribuição $\mathrm{N}(0,1)$ são $\mu_{X}=0$ e $\xi \phi_{X}^{-1}=1$, respectivamente. Utilizando a aproximação dada em (3.4) temos que

$$
\mathrm{E}(Y) \approx \frac{1}{1+e^{-\mu}}, \quad \operatorname{Var}(Y) \approx \frac{e^{-2 \mu}}{\phi^{2}\left(1+e^{-\mu}\right)^{4}} .
$$

Na Figura 3.2 estão expressos os gráficos da fdp da distribuição $\operatorname{LN}(\mu, \phi)$ para diferentes configurações de $\mu$ e $\phi$. As formas possíveis para esta distribuição são simétrica, assimétrica, bimodal, U, J e J invertido. Para forma simétrica deve-se ter $\mu=0$, enquanto assimétrica $\mu \neq 0$. O caso bimodal é bem limitado. Para forma de U é necessário $\mu=0.5$ e $\phi \leq 0.2$. Não conseguimos uma relação matemática para as formas $\mathrm{J}$ e $\mathrm{J}$ invertido.

\section{Distribuição logito t (LT)}

Se $X \sim \mathrm{T}(0,1, \nu)$ (t de Student padrão com $\nu$ graus de liberdade), com $f(x)=(1+$ $\left.x^{2} / \nu\right)^{-(\nu+1) / 2} \Gamma((\nu+1) / 2) /[\Gamma(\nu / 2) \sqrt{\nu \pi}]$, então dizemos que $Y$ dado em (3.3) tem distribuição logito $\mathrm{T}$ com parâmetros $\mu \in \mathbb{R}, \phi>0$ e graus de liberdade $\nu>0$, e escrevemos $Y \sim$ 


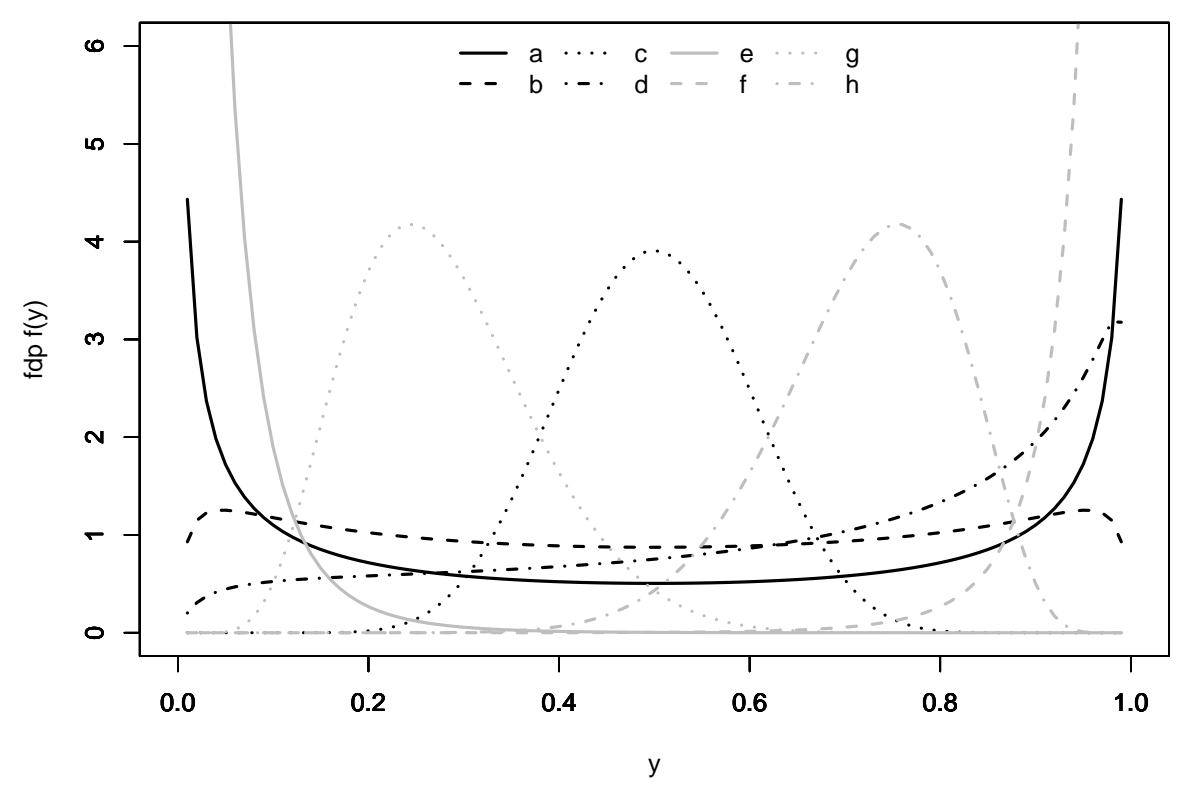

Figura 3.2: Funçôes densidade de probabilidade da distribuição $L N(\mu, \phi):(a)(\mu, \phi)=(0,0.1)$, (b) $(\mu, \phi)=(0,0.3),(c)(\mu, \phi)=(0,6),(d)(\mu, \phi)=(1,0.3),(e)(\mu, \phi)=(-3.5,1),(f)(\mu, \phi)=(3.5,1)$, (g) $(\mu, \phi)=(-1,4)$ e $(h)(\mu, \phi)=(1,4)$.

$\mathrm{LT}(\mu, \phi, \nu)$, e sua fdp é dada por

$$
f(y ; \mu, \phi, \nu)=\sqrt{\frac{\phi}{\pi \nu}} \frac{\Gamma((\nu+1) / 2)}{\Gamma(\nu / 2)}\left[1+\frac{\phi\left(y^{*}-\mu\right)^{2}}{\nu}\right]^{-(\nu+1) / 2} \frac{1}{y(1-y)}, \quad y \in(0,1),
$$

e sua fda é

$$
F(y ; \mu, \phi, \nu)=\sqrt{\frac{\phi}{\nu}}\left[y^{*}-\mu\left({ }_{2} F_{1}\left(\frac{1}{2} ; \frac{\nu+1}{2} ; \frac{3}{2} ; \frac{\phi\left(y^{*}-\mu\right)^{2}}{\nu}\right)-{ }_{2} F_{1}\left(\frac{1}{2} ; \frac{\nu+1}{2} ; \frac{3}{2} ; \frac{\phi \mu^{2}}{\nu}\right)\right)\right] \mathrm{B}^{-1}\left(\frac{\nu}{2} ; \frac{1}{2}\right),
$$

$y \in(0,1)$, em que ${ }_{2} F_{1}$ é a função hipergeométrica (Bateman et al., 1953) e B a função beta. Evidentemente, os quantis de $Y$ não têm forma fechada.

A média e a variância da distribuição $\mathrm{T}(0,1)$ são $\mu_{X}=0$ para $\nu>1$ e $\xi \phi_{X}^{-1}=\nu /(\nu-2)$ para $\nu>2$, respectivamente. Utilizando a aproximação dada em (3.4) temos que

$$
\mathrm{E}(Y) \approx \frac{1}{1+e^{-\mu}}, \quad \operatorname{Var}(Y) \approx \frac{\nu e^{-2 \mu}}{\left[\phi^{2}(\nu-2)\left(1+e^{-\mu}\right)^{4}\right.},
$$

se $\nu>2$.

A Figura 3.3 exibe esboços da fdp da distribuição $\operatorname{LT}(\mu, \phi, \nu)$ para alguns pares de parâmetros $\mu, \phi$ e $\nu$. A distribuição LT pode assumir todas as formas da distribuição LN, sob as mesmas condições desde que $\tau$ seja grande. Além disso, para $\tau$ pequeno temos os casos de caudas pesadas. 


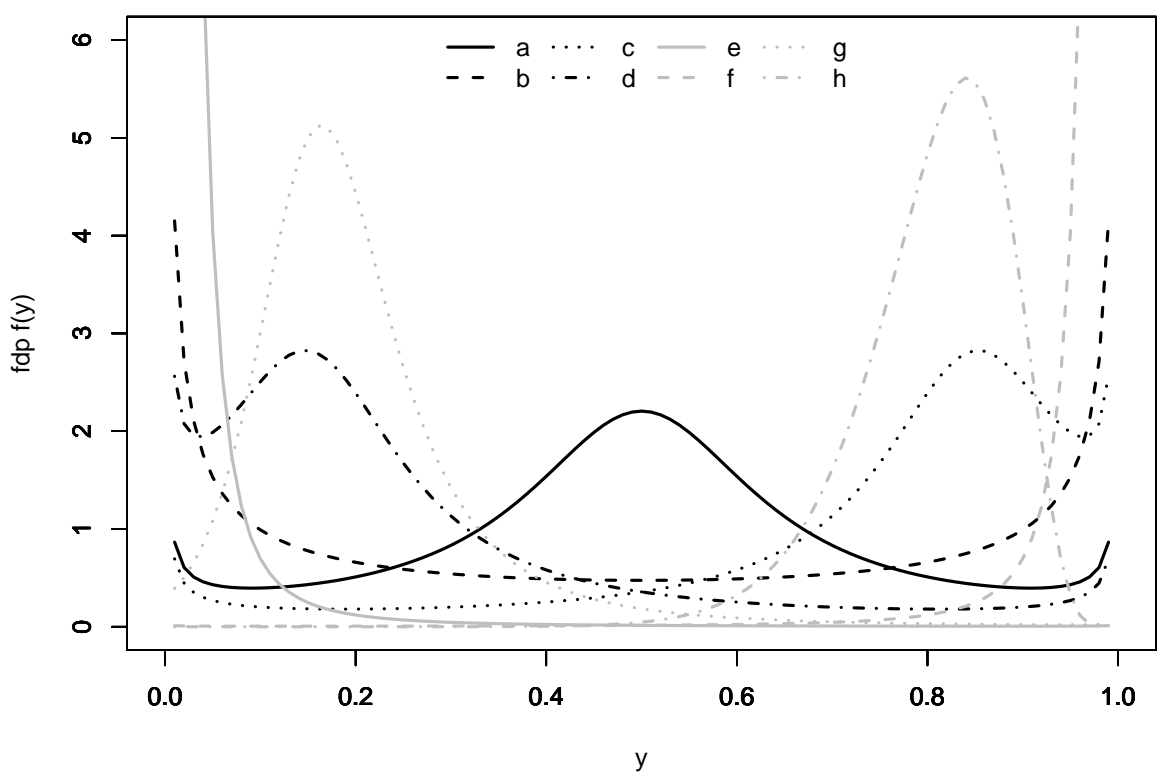

Figura 3.3: Funções densidade de probabilidade da distribuição $\operatorname{LT}(\mu, \phi, \nu):(a)(\mu, \phi, \nu)=(0,3,1)$, (b) $(\mu, \phi, \nu)=(0,0.1,4)$, (c) $(\mu, \phi, \nu)=(1.5,1.5,1)$, (d) $(\mu, \phi, \nu)=(-1.5,1.5,1)$, (e) $(\mu, \phi, \nu)=$ $(-4,1.5,4),(f)(\mu, \phi, \nu)=(4,1.5,4),(g)(\mu, \phi, \nu)=(-1.5,4,3)$ e (h) $(\mu, \phi, \nu)=(1.5,4,50)$.

\section{Distribuição logito exponencial potência (LEP)}

Se $X \sim \operatorname{PE}(0,1, \nu)$, com

$$
f(x)=\nu \sqrt{\phi} e^{-0.5|x / c|^{\nu}} /\left[c 2^{(1+1 / \nu)} \Gamma(1 / \nu)\right],
$$

em que $c=\sqrt{2^{-2 / \nu \Gamma(1 / \nu) / \Gamma(3 / \nu)}}$, dizemos que $Y$ dado em (3.3) tem distribuição logito exponencial potência com parâmetros $\mu \in \mathbb{R}, \phi>0$ e $\nu>0$, e escrevemos $Y \sim \operatorname{LEP}(\mu, \phi, \nu)$, com fdp dada por

$$
f(y ; \mu, \phi, \nu)=\frac{\nu \exp \left\{-\frac{1}{2}\left|\frac{\sqrt{\phi}\left(y^{*}-\mu\right)}{c}\right|^{\nu}\right\}}{c 2^{(1+1 / \nu)} \Gamma(1 / \nu)} \frac{\sqrt{\phi}}{y(1-y)}, \quad y \in(0,1) .
$$

A média e a variância da distribuição $\operatorname{EP}(0,1)$ são $\mu_{X}=0$ e $\xi \phi_{X}^{-1}=1$, respectivamente. Utilizando a aproximação dada em (3.4) temos que

$$
\mathrm{E}(Y) \approx \frac{1}{1+e^{-\mu}}, \quad \operatorname{Var}(Y) \approx \frac{e^{-2 \mu}}{\phi^{2}\left(1+e^{-\mu}\right)^{4}}
$$

A Figura 3.4 exibe gráficos da fdp da distribuição LEP para diferentes valores de $\mu, \phi$ e $\nu$. Quando $\nu=2$ temos o caso da distribuição LN, portanto é uma extensão da família de distribuições LN. A distribuição LEP é uma alternativa a distribuição LT que tem formas no sentido de comportar dados com cauda pesada. Na LEP é possível ter formas flexíveis em subintervalos do intervalo $(0,1)$.

No geral, todas as distribuições desta subseção podem assumir as formas simétrica, as- 


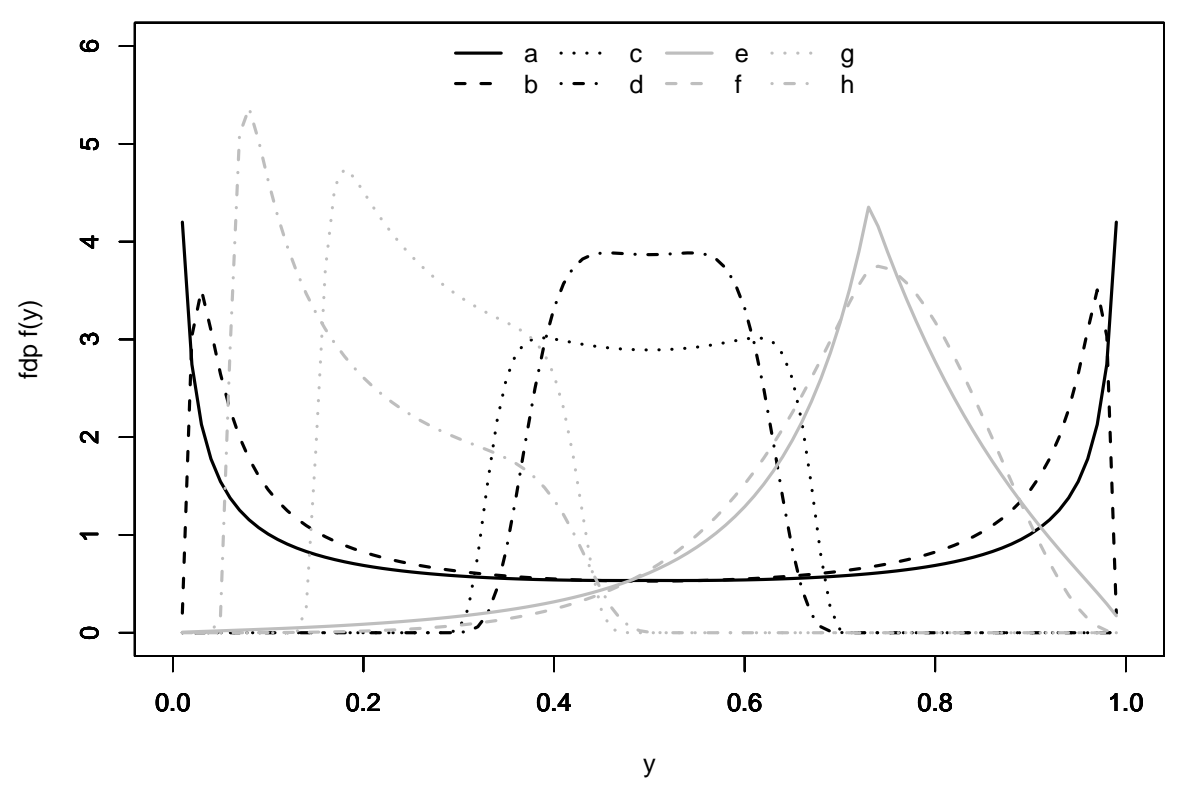

Figura 3.4: Funções densidade de probabilidade da distribuição $\operatorname{LEP}(\mu, \phi, \nu):(a)(\mu, \phi, \nu)=$ $(0,0.1,1.5)$, (b) $(\mu, \phi, \nu)=(0,0.1,10)$, (c) $(\mu, \phi, \nu)=(0,3,10)$, (d) $(\mu, \phi, \nu)=(0,6,5)$, (e) $(\mu, \phi, \nu)=(1,3,1),(f)(\mu, \phi, \nu)=(1,3,1.5),(g)(\mu, \phi, \nu)=(-1,3,10)$ e $(h)(\mu, \phi, \nu)=(-1.5,1,10)$.

simétrica, U, J e J invertido, sendo que a distribuição LL é a única que assume a forma uniforme. Além disso, as distribuições LT e LEP conseguem outras formas que não sejam as citadas no início do parágrafo. Entretanto, essas formas são restritas, como por exemplo, na distribuição LT as modas das formas bimodais têm uma certa restrição, ou seja, não podem ser em qualquer posição do intervalo $(0,1)$. Sendo assim, é mais apropriado utilizar uma mistura de distribuições para obter multimodalidade.

\subsubsection{Classe logito skew}

Nesta subseção apresentamos as distribuições logito com $X$ tendo distribuição na classe skew, expressando as fdp's e fda's, assim como a média e variância utilizando a aproximação por expansão em série de Taylor, suas formas e alguns comentários que julgamos relevantes. Se $\lambda=0$ temos as distribuições da Subseção 3.2.1.

\section{Distribuição logito skew logística (LSL)}

Se $X \sim \operatorname{SL}(0,1, \lambda)$, com $f(x)=2 g_{X_{0}}(x) G_{X_{0}}(\lambda x)$, em que $X_{0} \sim \mathrm{L}(0,1)$ e $\lambda \in \mathbb{R}$, então dizemos que $Y$ dado em (3.3) tem distribuição logito skew logística com parâmetro de locação $\mu \in \mathbb{R}$, precisão $\phi>0$ e assimetria $\lambda \in \mathbb{R}$, e escrevemos $Y \sim \operatorname{LSL}(\mu, \phi, \lambda)$. Sua fdp é

$$
f(y ; \mu, \phi, \lambda)=\frac{2 \sqrt{\phi} e^{-\sqrt{\phi}\left(y^{*}-\mu\right)}}{\left[1+e^{-\sqrt{\phi}\left(y^{*}-\mu\right)}\right]^{2}[y(1-y)]\left[1+e^{-\lambda \sqrt{\phi}\left(y^{*}-\mu\right)}\right]}, \quad y \in(0,1) .
$$

Para mais detalhes sobre a distribuição skew logística ver Gupta et al. (2002) e Nadarajah (2009). 
Nadarajah (2009) apresenta os quatro primeiros momentos da distribuição skew logística. Seja

$$
\kappa(a, q)=\sum_{j=0}^{\infty} \frac{1}{(q+j)^{a}}
$$

$\mathrm{e}$

$$
\varsigma(j, k)=\kappa\left(k, \frac{1+2 \lambda+j}{2 \lambda}\right)-\kappa\left(k, \frac{1+\lambda+j}{2 \lambda}\right) .
$$

Temos que a média e a variância da distribuição skew logística padrão são

$$
\mu_{X}=\frac{1}{\lambda^{2}} \sum_{j=0}^{\infty}(-1)^{j}(j+1) \varsigma(j, 2), \quad \xi \phi_{X}^{-1}=\frac{\pi}{3}-\left(\mu_{X}\right)^{2},
$$

respectivamente.

Utilizando a aproximação dada em (3.4) temos que

$$
\mathrm{E}(Y) \approx \frac{1 / \lambda^{2} \sum_{j=0}^{\infty}(-1)^{j}(j+1) \varsigma(j, 2) e^{-\mu}+\sqrt{\phi}\left(1+e^{-\mu}\right)}{\sqrt{\phi}\left(1+e^{-\mu}\right)^{2}}
$$

$\mathrm{e}$

$$
\operatorname{Var}(Y) \approx\left[\frac{\pi}{3}-\left(\mu_{X}\right)^{2}\right]\left[\frac{e^{-\mu}}{\phi\left(1+e^{-\mu}\right)^{2}}\right]^{2}
$$

A Figura 3.5 mostra gráficos de fdp da distribuição $\operatorname{LSL}(\mu, \phi, \lambda)$ com várias configurações de $\mu, \phi$ e $\lambda$. Quando $\lambda=0$ temos a distribuição LL. Nesta distribuição há uma vasta gama de possibilidades de formas.

\section{Distribuição logito skew normal (LSN)}

Se $X \sim \operatorname{SN}(0,1, \lambda)$, com $f(x)=2 g_{X_{0}}(x) G_{X_{0}}(\lambda x)$, em que $X_{0} \sim \mathrm{N}(0,1)$ e $\lambda \in \mathbb{R}$, então dizemos que $Y$ dado em (3.3) tem distribuição logito skew normal com parâmetro de locação $\mu \in \mathbb{R}$, precisão $\phi>0$ e assimetria $\lambda \in \mathbb{R}$, e escrevemos $Y \sim \operatorname{LSN}(\mu, \phi, \lambda)$. Sua fdp é

$$
f(y ; \mu, \phi, \lambda)=\frac{\sqrt{2 \phi} e^{-\phi\left(y^{*}-\mu\right)^{2}} \Phi\left(-\lambda \sqrt{\phi}\left(y^{*}-\mu\right)\right)}{\sqrt{\pi} y(1-y)}, \quad y \in(0,1) .
$$

Para mais detalhes sobre a distribuição skew normal ver Azzalini (1985) e Gupta et al. (2002).

A média e a variância da distribuição skew normal padrão são

$$
\mu_{X}=\delta \sqrt{\frac{2}{\pi}}, \quad \xi \phi_{X}^{-1}=1-\frac{2 \delta^{2}}{\pi},
$$

com $\delta=\lambda / \sqrt{1+\lambda^{2}}$, respectivamente.

Utilizando a aproximação dada em (3.4) temos que

$$
\mathrm{E}(Y) \approx \frac{\delta \sqrt{2 / \pi} e^{-\mu}+\sqrt{\phi}\left(1+e^{-\mu}\right)}{\sqrt{\phi}\left(1+e^{-\mu}\right)^{2}}, \quad \operatorname{Var}(Y) \approx\left[1-\frac{2 \delta^{2}}{\pi}\right]\left[\frac{e^{-\mu}}{\phi\left(1+e^{-\mu}\right)^{2}}\right]^{2} .
$$




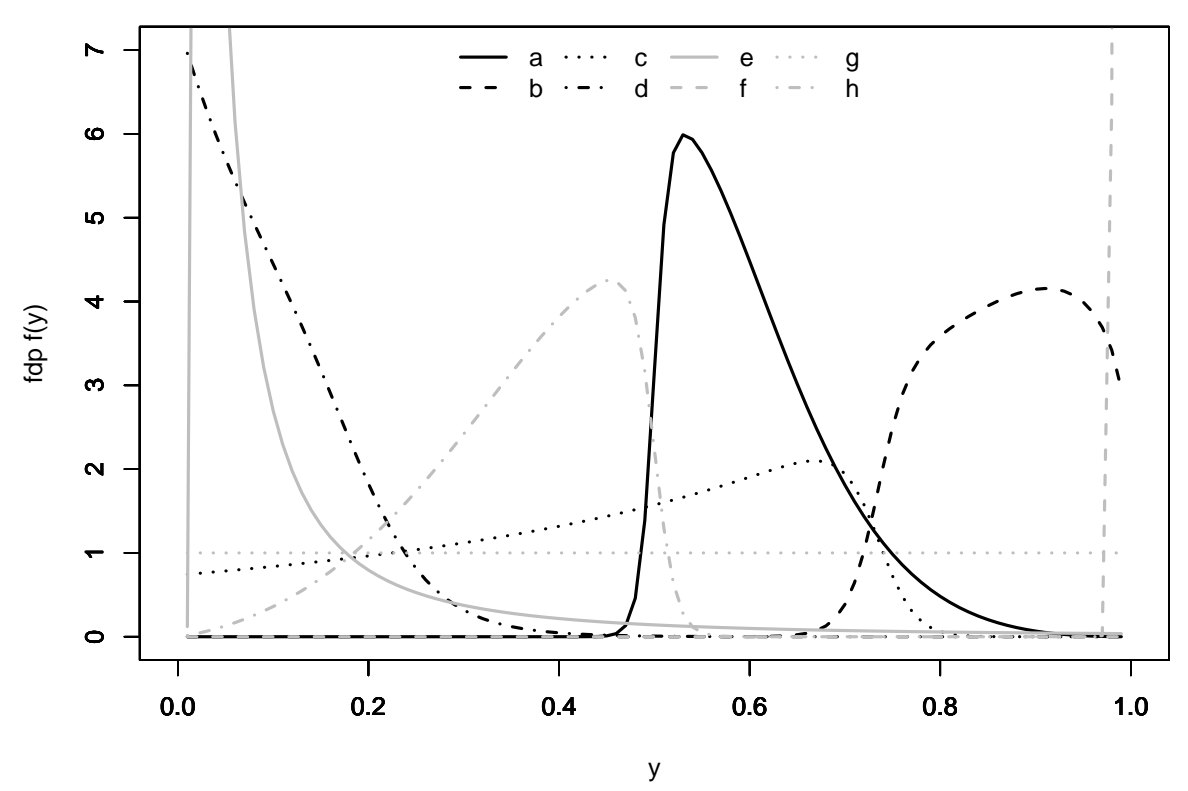

Figura 3.5: Funções densidade de probabilidade da distribuição $\operatorname{LSL}(\mu, \phi, \lambda):$ (a) $(\mu, \phi, \lambda)=$ $(0,10,-10)$, (b) $(\mu, \phi, \lambda)=(1,1.5,-10)$, (c) $(\mu, \phi, \lambda)=(1,1,10)$, (d) $(\mu, \phi, \lambda)=(-1.3,1,4)$, (e) $(\mu, \phi, \lambda)=(-4,1,-10)$, (f) $(\mu, \phi, \lambda)=(4,2,-10),(g)(\mu, \phi, \lambda)=(0,1,0)$ e $(h)(\mu, \phi, \lambda)=(0,5,10)$.

Na Figura 3.6 estão expressos os gráficos da fdp da distribuição $\operatorname{LSN}(\mu, \phi, \lambda)$ para diferentes configurações de $\mu, \phi$ e $\lambda$. Quando $\lambda=0$ temos a distribuição LN. Assim como a distribuição LSL, nesta distribuição é possível obter uma grande variedade de formas assimétricas.

\section{Distribuição logito skew t (LST)}

Se $X \sim \mathrm{ST}(0,1, \nu, \lambda)$, com $f(x)=2 g_{X_{0}}(x) G_{X_{0}}(\lambda x)$, em que $X_{0} \sim \mathrm{T}(0,1, \nu)$ e $\lambda \in \mathbb{R}$, então dizemos que $Y$ dado em (3.3) tem distribuição logito skew t com parâmetro de locação $\mu \in \mathbb{R}$, precisão $\phi>0$, graus de liberdade $\nu>0$ e assimetria $\lambda \in \mathbb{R}$, e escrevemos $Y \sim$ $\operatorname{LST}(\mu, \phi, \nu, \lambda)$. Sua fdp é

$$
f(y ; \mu, \phi, \nu, \lambda)=\sqrt{\frac{\phi}{\pi \nu}} \frac{\Gamma((\nu+1) / 2)}{\Gamma(\nu / 2)}\left[1+\frac{\phi\left(y^{*}-\mu\right)^{2}}{\nu}\right]^{-(\nu+1) / 2} \frac{2 F(\lambda y ; \mu, \phi, \nu)}{y(1-y)}, \quad y \in(0,1) .
$$

Para mais detalhes sobre a distribuição skew t ver Gupta et al. (2002) e Azzalini e Capitanio (2003). A distribuição logito skew t foi introduzida e aplicada à estimação de quantis em Hossain et al. (2016).

A Figura 3.7 exibe esboços da fdp da distribuição $\operatorname{LST}(\mu, \phi, \nu, \lambda)$ para alguns pares de parâmetros $\mu, \phi, \nu$ e $\lambda$. Quando $\lambda=0$ temos como caso particular a distribuição LT. Assim como as distribuições LSL e LSN, esta distribuição comporta bem os casos de assimetria. Além disso, permite formas que não são possíveis nas distribuições anteriormente apresentadas, que é o caso das configurações $(c)$ e (d). 


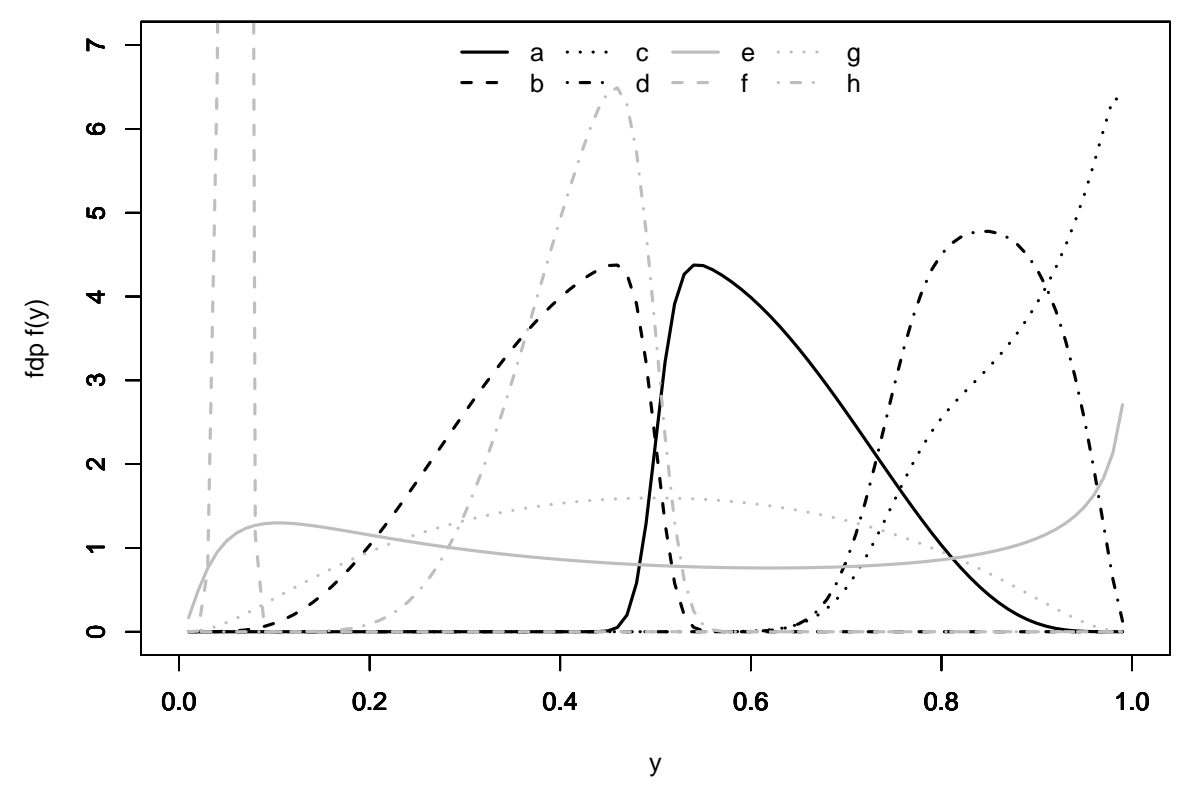

Figura 3.6: Funções densidade de probabilidade da distribuição $\operatorname{LSN}(\mu, \phi, \lambda):$ (a) $(\mu, \phi, \lambda)=$ $(0,2,10)$, (b) $(\mu, \phi, \lambda)=(0,2,-10)$, (c) $(\mu, \phi, \lambda)=(1,0.3,8),(d)(\mu, \phi, \lambda)=(1,1,5),(e)(\mu, \phi, \lambda)=$ $(-2.1,0.1,3),(f)(\mu, \phi, \lambda)=(-2.5,10,-10),(g)(\mu, \phi, \lambda)=(0,1,0) e(h)(\mu, \phi, \lambda)=(0,5,-5)$.

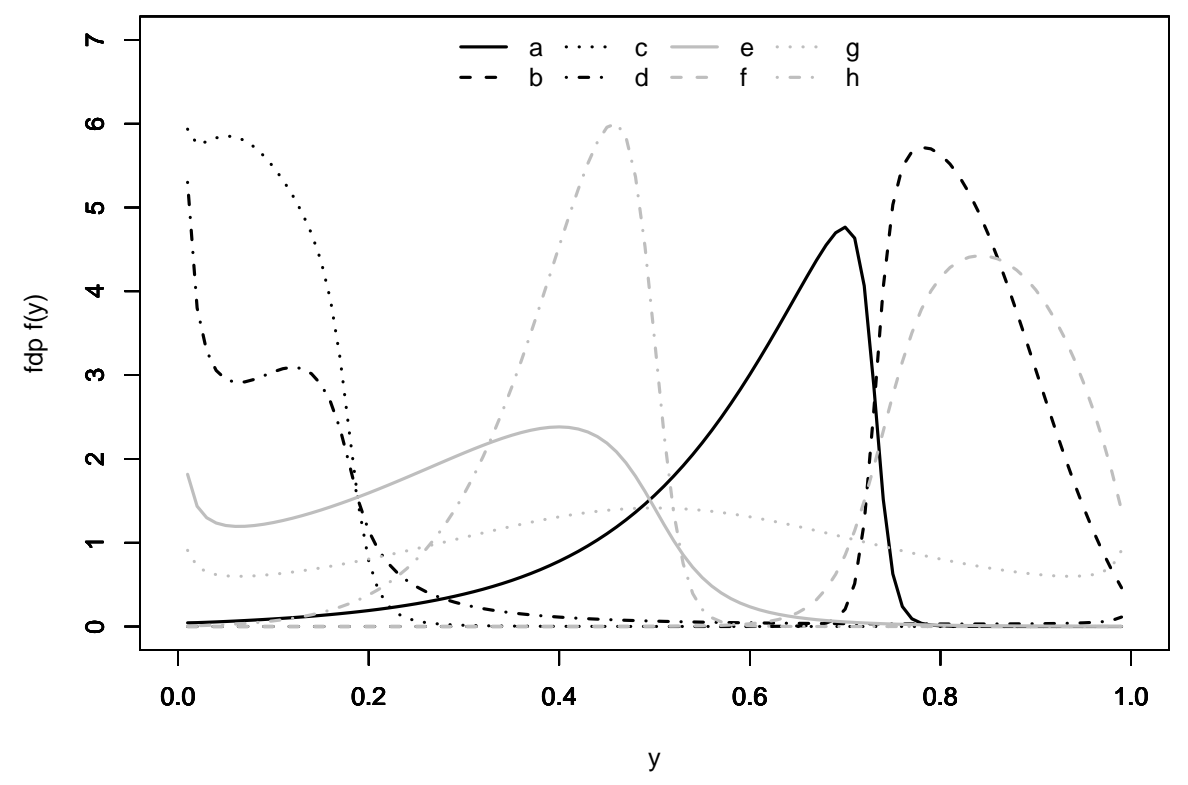

Figura 3.7: Funçôes densidade de probabilidade da distribuição $\operatorname{LST}(\mu, \phi, \nu, \lambda):$ (a) $(\mu, \phi, \nu, \lambda)=$ $(1,2,5,-10)$, (b) $(\mu, \phi, \nu, \lambda)=(1,2,5,10)$, (c) $(\mu, \phi, \nu, \lambda)=(-1.5,0.7,3,-10)$, (d) $(\mu, \phi, \nu, \lambda)=$ $(-1.5,1,0.5,-6)$, (e) $(\mu, \phi, \nu, \lambda)=(0,1,2,-5)$, (f) $(\mu, \phi, \nu, \lambda)=(1,1,6,5), \quad(g)(\mu, \phi, \nu, \lambda)=$ $(0,1,2,0) e(h)(\mu, \phi, \nu, \lambda)=(0,5,6,-5)$.

\section{Distribuição logito skew exponencial potência (LSEP)}

Se $X \sim \operatorname{SEP}(0,1, \nu, \lambda)$, com $f(x)=2 g_{X_{0}}(x) G_{X_{0}}(\lambda x)$, em que $X_{0} \sim \operatorname{EP}(0,1, \nu)$ e $\lambda \in \mathbb{R}$, então dizemos que $Y$ dado em (3.3) tem distribuição logito skew exponencial potencia com 
parâmetro de locação $\mu \in \mathbb{R}$, precisão $\phi>0$, curtose $\nu>0$ e assimetria $\lambda \in \mathbb{R}$, e escrevemos $Y \sim \operatorname{LSEP}(\mu, \phi, \nu, \lambda)$. A fdp de $Y$ é dada por

$$
f(y ; \mu, \phi, \nu \lambda)=\frac{2 \sqrt{\phi} \nu \exp \left\{-\frac{1}{2}\left|\frac{\sqrt{\phi}\left(y^{*}-\mu\right)}{c}\right|^{\nu}\right\} \Phi_{E P_{0}}\left(\operatorname{sinal}(z)|z|^{\nu / 2} \lambda(2 / \nu)^{1 / 2}\right)}{c 2^{(1+1 / \nu)} \Gamma(1 / \nu)[y(1-y)]}, \quad y \in(0,1),
$$

em que $c=\sqrt{2^{-2 / \nu \Gamma(1 / \nu) \Gamma(3 / \nu)}}$ e $z=\sqrt{\phi}\left(y^{*}-\mu\right)$. Para mais detalhes sobre a distribuição skew exponencial potência ver Azzalini (1986) e DiCiccio e Monti (2004).

A Figura 3.8 exibe gráficos da fdp da distribuição LSEP para diferentes valores de $\mu, \phi, \nu$ e $\lambda$. Quando $\lambda=0$ temos o caso da distribuição LEP. Assim como a distribuição LST, esta distribuição permite formas mais exóticas em relação às distribuições LSL e LSN. De certa maneira, algumas dessas formas parecem pouco plausíveis para modelar dados reais.

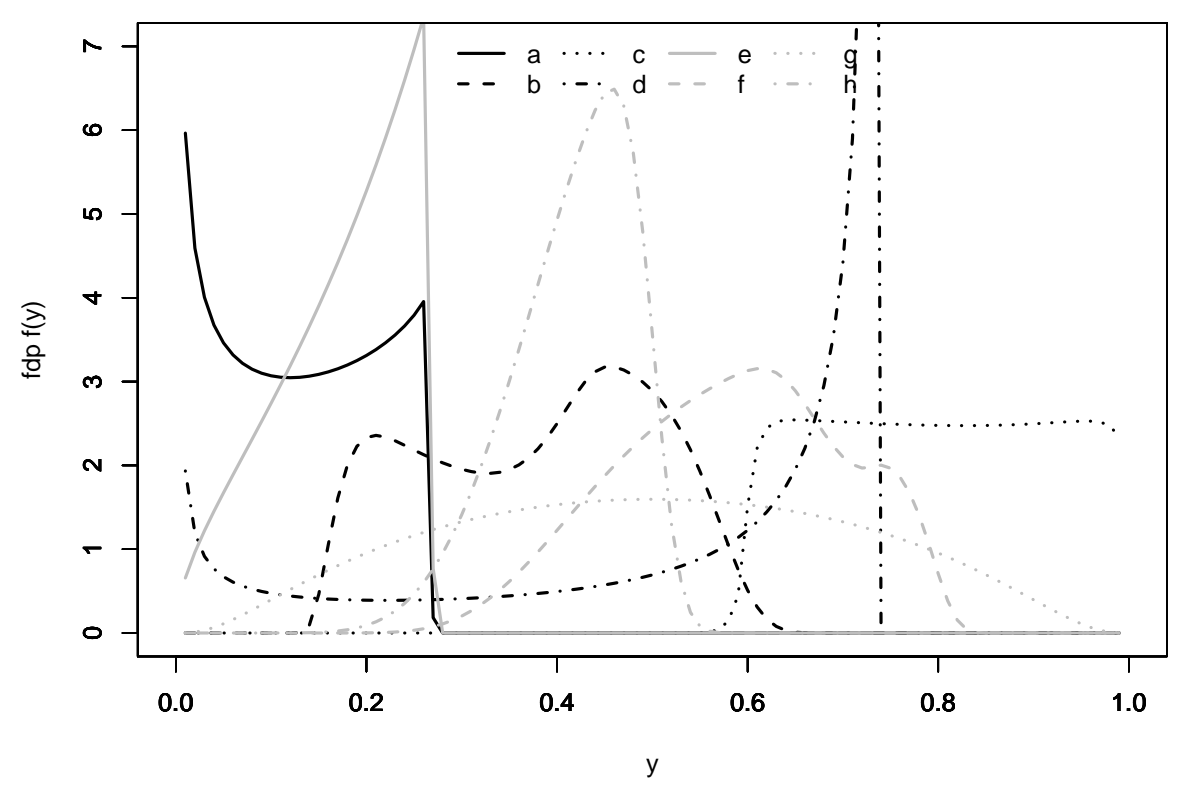

Figura 3.8: Funções densidade de probabilidade da distribuição $\operatorname{LSEP}(\mu, \phi, \nu, \lambda):($ a) $(\mu, \phi, \nu, \lambda)=$ $(-1,0.5,0.8,-10), \quad$ (b) $(\mu, \phi, \nu, \lambda)=(-1,1,8,10), \quad$ (c) $(\mu, \phi, \nu, \lambda)=(0.4,0.5,1.4,10), \quad$ (d) $(\mu, \phi, \nu, \lambda)=(1,2,0.2,-7.5)$, (e) $(\mu, \phi, \nu, \lambda)=(-1,2.3,1,-10)$, (f) $(\mu, \phi, \nu, \lambda)=(1,1,4,-10)$, (g) $(\mu, \phi, \nu, \lambda)=(0,1,2,0)$ e $(h)(\mu, \phi, \nu, \lambda)=(0,5,2,-5)$.

\subsection{Estimação}

Nesta seção será apresentado o processo de estimação dos parâmetros da classe logito e logito skew, já que para as distribuições apresentadas no Capítulo 2 já há uma vasta literatura com métodos de estimação assim como vários algoritmos implementados. Vários métodos podem ser utilizados para estimar os parâmetros. Os mais utilizados são os métodos de mínimos quadrados, momentos e máxima verossimilhança. Daremos destaque ao método da máxima verossimilhança pois, além de ser o método mais utilizado, é o que também tem uma quantidade significativa de propriedades úteis para o processo inferencial. 


\subsubsection{Estimação na classe logito}

A estimação dos parâmetros dessa classe foi estudada por Lemonte e Bazán (2015). Apresentaremos os resultados obtidos por eles para o caso da estimação pelo método da máxima verossimilhança.

Sejam $y_{1}, \ldots, y_{n}$ uma amostra aleatória de $Y \sim \operatorname{Logito}(\mu, \phi ; g)$ com fdp $f_{Y}$ dada em (3.6). O logaritmo da função de verossimilhança é dado por

$$
l=l(\mu, \phi)=\frac{n}{2} \log \phi+\sum_{i=1}^{n} \log g\left(y_{0 i}^{* 2}\right)-\sum_{i=1}^{n} \log y_{i}\left(1-y_{i}\right),
$$

em que $y_{0}^{*}=\sqrt{\phi}\left(y^{*}-\mu\right)$

Assumindo que $g$ é diferenciável então as funções escore de $\mu$ e $\phi$ são

$$
U_{\mu}(\mu, \phi)=\frac{\partial l}{\partial \mu}=-2 \sqrt{\phi} \sum_{i=1}^{n} y_{0 i}^{*} W_{g}\left(y_{0 i}^{* 2}\right)
$$

e

$$
U_{\phi}(\mu, \phi)=\frac{\partial l}{\partial \phi}=\frac{n}{2 \phi}+\frac{1}{\phi} \sum_{i=1}^{n} y_{0 i}^{* 2} W_{g}\left(y_{0 i}^{* 2}\right),
$$

respectivamente, em que $W_{g}(u)=g^{\prime}(u) / g(u)$. Na Tabela (3.1) estão as expressões de $W_{g}$ para as distribuições com as quais estamos trabalhando. Para obter as estimativas de máxima verossimilhança é necessário encontrar as raízes do sistema de equações $U_{\mu}(\mu, \phi)=$ $U_{\phi}(\mu, \phi)=0$. Como são funções não-lineares, então é necessário utilizar algum método numérico como Newton-Raphson. Um outro aspecto é que $W_{g}$ pode depender de quantidades desconhecidas, como é o caso do parâmetro de graus de liberdade na distribuição t e do parâmetro de potência da distribuição exponencial potência. Nesse caso, pode-se estabelecer

\begin{tabular}{|c|c|c|c|c|}
\hline & Logística & Normal & $\mathrm{t}$ & Exponencial potência \\
\hline \multirow{2}{*}{$g(u)$} & $e^{-\sqrt{u}}$ & $e^{-u / 2}$ & $\left.\mathrm{~B}^{-1}(1 / 2 ; \nu / 2){ }_{1} \quad u\right\rceil^{-(\nu+1) / 2}$ & $\nu e^{-0.5\left(u / c^{2}\right)^{\nu / 2}}$ \\
\hline & $\overline{\left(1+e^{-\sqrt{u}}\right)^{2}}$ & $\overline{\sqrt{2 \pi}}$ & {$\left[1+\frac{u}{\nu}\right]^{-(\nu+1) / 2}$} & $\overline{c 2^{(1+1 / \nu)} \Gamma(1 / \nu)}$ \\
\hline \multirow{2}{*}{$W_{g}(u)$} & $1-e^{-\sqrt{u}}$ & 1 & $\nu+1$ & $\nu(u)^{\nu / 2-1}$ \\
\hline & $\overline{2 \sqrt{u}\left(1+e^{-\sqrt{u}}\right)}$ & $\overline{2}$ & $\overline{2(\nu+u)}$ & $\overline{4}\left(\overline{c^{2}}\right)$ \\
\hline
\end{tabular}
um valor fixado (conhecido) para o parâmetro de graus de liberdade.

Tabela 3.1: Funções $g(u)$ e $W_{g}(u)$ para diferentes distribuições.

Uma segunda maneira de estimar os parâmetros pelo método da máxima verossimilhança é fazer uma transformação inversa, ou seja, ao invés de estimar os parâmetros com os dados na escala original estimam-se os parâmetros na escala transformada. Para isso basta realizar a seguinte transformação

$$
\mu+\frac{X}{\sqrt{\phi}}=\log \left\{\frac{Y}{1-Y}\right\}
$$

dessa maneira $\mu+X / \sqrt{\phi} \sim \mathrm{S}(\mu, \phi ; g)$. 


\subsubsection{Estimação na classe logito skew}

Sejam $y_{1}, \ldots, y_{n}$ uma amostra aleatória de $Y \sim \operatorname{LogitoS}(\mu, \phi, \lambda ; \mathrm{g})$ com fdp $f_{Y}$ dada por (3.6) sendo que a v.a. $X$ tem fdp dada por (3.1) e a v.a. $X_{0}$ tem a mesma fdp $\operatorname{com} \lambda=0$, ou seja, tem uma distribuição simétrica. O logaritmo da função de verossimilhança é dado por

$$
l=l(\mu, \phi, \lambda)=\frac{n}{2} \log \phi+\sum_{i=1}^{n} \log g\left(y_{0 i}^{* 2}\right)-\sum_{i=1}^{n} \log y_{i}\left(1-y_{i}\right)+\sum_{i=1}^{n} \zeta_{0}\left(\lambda y_{0 i}^{*}\right),
$$

em que $y_{0}^{*}=\sqrt{\phi}\left(y^{*}-\mu\right)$, com $y^{*}=\log [y /(1-y)]$ e $\zeta_{0}(\cdot)=\log \left(2 \Phi_{X_{0}}(\cdot)\right)$, com $\Phi_{X_{0}}$ sendo a fda de $X_{0}$.

A função $g$ é a mesma apresentada na Subseção 3.3.1. As funções escores de $\mu, \phi$ e $\lambda$ são

$$
U_{\mu}(\mu, \phi)=\frac{\partial l}{\partial \mu}=-2 \sqrt{\phi} \sum_{i=1}^{n} y_{0 i}^{*} W_{g}\left(y_{0 i}^{* 2}\right)-\lambda \sqrt{\phi} \sum_{i=1}^{n} \zeta_{1}\left(\lambda y_{0 i}^{*}\right),
$$

em que $\zeta_{1}(\cdot)=f(\cdot) / \Phi(\cdot)$,

$$
U_{\phi}(\mu, \phi)=\frac{\partial l}{\partial \phi}=\frac{n}{2 \phi}+\frac{1}{\phi} \sum_{i=1}^{n} y_{0 i}^{* 2} W_{g}\left(y_{0 i}^{* 2}\right)+\frac{\lambda}{2 \phi} \sum_{i=1}^{n} y_{0 i}^{*} \zeta_{1}\left(\lambda y_{0 i}^{*}\right)
$$

$\mathrm{e}$

$$
U_{\lambda}(\mu, \phi)=\frac{\partial l}{\partial \lambda}=\sum_{i=1}^{n} y_{0 i}^{*} \zeta_{1}\left(\lambda y_{0 i}^{*}\right)
$$

respectivamente. De (3.10) e (3.11) temos que $\widehat{\mu}$ e $\widehat{\phi}$, as estimativas de máxima verossimilhança de $\mu$ e $\phi$, devem satisfazer a equação

$$
\widehat{\phi}=-\frac{n}{2}\left[\sum_{i=1}^{n}\left(y^{*}-\widehat{\mu}\right)^{2} W_{g}\left(\widehat{y}_{0 i}^{*}\right)\right] .
$$

Novamente como as equações $U_{\mu}(\mu, \phi)=U_{\phi}(\mu, \phi)=U_{\lambda}(\mu, \phi)=0$ são não-lineares é necessário utilizar métodos numéricos. Uma possível solução para esse problema é fazer a transformação inversa

$$
\mu+\frac{X}{\sqrt{\phi}}=\log \left\{\frac{Y}{1-Y}\right\}
$$

em que $\mu+X / \sqrt{\phi} \sim \operatorname{Skew}(\mu, \phi, \lambda ; g)$. Dessa forma, utilizam-se os métodos existentes para estimar os parâmetros por máxima verossimilhança para os dados transformados. Além do pacote gamlss, o pacote sn de Azzalini (2017) estima os parâmetros das distribuições skew normal e skew t. 


\section{Capítulo 4}

\section{Aplicação}

Nos Capítulos 2 e 3 foram apresentadas várias distribuições de probabilidade que podem ser utilizadas para modelar dados com distribuição contínua e suporte no intervalo $(0,1)$. Neste capítulo apresentamos alguns conjuntos de dados com essa característica. Além disso, buscamos dados das mais diversas formas para realizar um estudo de qual ou quais distribuições apresentam uma maior flexibilidade em termos práticos para modelar esses dados.

Utilizamos o software R (R Core Team, 2017) para estimar os parâmetros das distribuições pelo método de máxima verossimilhança. Para a distribuição beta, Kumaraswamy, simplex, gama unitária, beta retangular e logito skew logística utilizamos a função optim com derivadas numéricas, enquanto que, para as demais distribuições, usamos a função gamlss do pacote gamlss (Stasinopoulos et al., 2017). Para a distribuição simplex estimamos $\sigma^{2}=1 / \phi$ e pelo princípio da invariância obtivemos a estimativa de $\phi$.

Para escolher qual distribuição melhor se ajusta aos dados é necessário utilizar algum critério. Sendo assim, utilizaremos quatro critérios para escolher qual distribuição melhor se ajusta a cada variável do conjunto de dados da aplicação. Os critérios utilizados são apresentados a seguir.

\section{AIC}

Akaike (1973) propôs utilizar a divergência de Kullback-Leibler (K-L) para selecionar modelos. Entretanto, para calcular a divergência de K-L é necessário o conhecimento do verdadeiro modelo $f$. Akaike estima a divergência de K-L através da função log-verossimilhança no ponto máximo menos $k$, que é o número de parâmetros do modelo. A constante $k$ para grandes amostras é uma boa aproximação para a quantidade desconhecida da divergência.

Suponha que temos um conjunto de dados e um modelo estatístico. Seja $k$ o número de parâmetros do modelo e $\widehat{L}$ o valor máximo da função de verossimilhança. Então o critério de informação de Akaike (AIC) do modelo é dado por

$$
\mathrm{AIC}=-2 \log (\widehat{L})+2 k .
$$

A diferença $\log (\widehat{L})-k$ é multiplicada por -2 por questões práticas. Dado um conjunto de modelos estatísticos, o modelo escolhido é o que apresenta menor AIC. 


\section{$\mathrm{BIC}$}

Um outro critério de seleção de modelos foi proposto por Schwarz (1978) e é conhecido como critério de informação bayesiana (BIC). É bem similar ao AIC, sendo que neste critério há uma penalização maior aos modelos que têm um maior número de parâmetros. Sua expressão é dada por

$$
\mathrm{BIC}=-2 \log (\widehat{L})+k \log n,
$$

ou seja, $\mathrm{BIC}=\mathrm{AIC}+k(\log n-2)$. Nota-se que o BIC é maior que o AIC para $n \geq 8$. Neste critério o melhor modelo é aquele que apresenta o menor BIC.

\section{Evidência empírica $\Delta$}

Na prática selecionam-se os modelos com menores AIC e BIC. Mas, dentre todos os modelos, aquele que apresentar o menor AIC ou BIC é de fato melhor que os outros? $\mathrm{Ou}$ seja, quão melhor é em detrimento aos demais modelos? Ainda, esse modelo é de fato uma boa aproximação para a realidade? Lembramos que estamos apenas comparando modelos.

Nesse sentido, Burnham e Anderson (2002) apresentam uma medida que quantifica a diferença entres os modelos. Essa medida é dada por

$$
\Delta_{m}=\mathrm{AIC}_{m}-\mathrm{AIC}_{\min }
$$

em que $\mathrm{AIC}_{m}$ é o valor do critério de AIC para o modelo $m$, com $m=1, \cdots, T$, e $T$ é o número de modelos considerados, e $\mathrm{AIC}_{\min }=\min \left\{\mathrm{AIC}_{m}\right\}$. Se $\Delta_{m} \in[0,2]$ então o modelo $m$ é considerado plausível, caso contrário não é um modelo aceitável. Possivelmente outros valores de corte podem ser utilizados ou podem ser criadas categorias de plausibilidade. Segundo Burnham e Anderson (2002), modelos com $\Delta_{m}>10$ podem ser descartados das análises; esses modelos falham em explicar algumas variações explicáveis.

Para quantificar essa diferença Burnham e Anderson (2002) propõem duas medidas: a razão da evidência,

$$
\mathrm{RE}_{\mathrm{m}}=\exp \left(\Delta_{m} / 2\right)
$$

(note que $\mathrm{RE}_{\mathrm{m}}=1$ para o modelo com menor $\mathrm{AIC}$ e $\mathrm{RE}_{\mathrm{m}}>1$ para os demais modelos), e o peso do modelo

$$
w_{\mathrm{m}}=\frac{\exp \left(-\Delta_{m} / 2\right)}{\sum_{i=m}^{T} \exp \left(-\Delta_{m} / 2\right)},
$$

que é interpretado como a probabilidade do modelo $m$ ser o melhor modelo.

Analogamente, difine-se peso do modelo baseado em BIC. Denotaremos os pesos por $w_{\mathrm{AIC}}$ e $w_{\mathrm{BIC}}$ quando utilizamos o AIC e BIC respectivamente.

\section{TRVG $V_{\text {RV }}$}

Uma outra forma de selecionar o melhor modelo é utilizar o teste de razão de verossimilhanças generalizado (TRVG) proposto por Vuong (1989). Para testar o modelo $g$ versus o modelo $f$ a estatística de teste é dada por

$$
V_{\mathrm{RV}}=\frac{l^{f}(\widehat{\theta})-l^{g}(\widehat{\gamma})}{\sqrt{n} \widehat{w}}=\frac{1}{\sqrt{n} \widehat{w}} \sum_{i=1}^{n} \log \frac{f\left(y_{i} ; \widehat{\theta}\right)}{g\left(y_{i} ; \widehat{\gamma}\right)},
$$


em que $l^{f}(\widehat{\theta})$ é a log-verossimilhança do modelo $f$ avaliada no estimador de máxima verossimilhança de $\theta$, que é o vetor de parâmetros do modelo $f, l^{g}(\widehat{\gamma})$ é a log-verossimilhança do modelo $g$ avaliada no estimador de máxima verossimilhança de $\gamma$, que é o vetor de parâmetros do modelo $g$, e

$$
\widehat{w}=\frac{1}{n} \sum_{i=1}^{n}\left\{\log \left[\frac{f\left(y_{i} ; \widehat{\theta}\right)}{g\left(y_{i} ; \widehat{\gamma}\right)}\right]\right\}^{2}-\left\{\frac{1}{n} \sum_{i=1}^{n} \log \left[\frac{f\left(y_{i} ; \widehat{\theta}\right)}{g\left(y_{i} ; \widehat{\gamma}\right)}\right]\right\}^{2} .
$$

A estatística $V_{\mathrm{RV}}$ mede a distância entre dois modelos em termos da discrepância de K-L e assintoticamente segue uma distribuição normal padrão sob a hipótese nula (Vuong, 1989). A hipótese nula é que $g$ é o modelo correto e a hipótese alternativa é a de que $f$ é o modelo correto. Valores grandes de $V_{\mathrm{RV}}$ dão evidências em favor de $f$ contra $g$. Se o p-valor for menor que o nível de significância então escolhemos o modelo $f$, caso contrário não há evidências suficientes contra o modelo $g$. Neste caso p-valor $=p_{V}=1-\Phi\left(V_{\mathrm{RV}}\right)$, em que $\Phi(t)$ é a fda da distribuição $\mathrm{N}(0,1)$ no ponto $t$.

Para nossa aplicação utilizamos um conjunto de dados de World Bank (2017). O World Bank ou Banco Mundial não é propriamente um banco no sentido comum. É um grupo formado por cinco instituições que têm como objetivo reduzir a extrema pobreza e promover a prosperidade. O Banco Mundial é uma fonte de assistência financeira e técnica aos países em desenvolvimento. Fundado em 1944, o grupo tem sede em Washington, DC e tem 10 mil funcionários em mais de 120 escritórios em todo o mundo. Para mais informações acessar World Bank.

Selecionamos as seguintes variáveis: exportações de alta tecnologia (proporção das exportações de manufaturados), despesas em educação (proporção da despesa total do governo), terra arável (proporção da área da terra), serviços (proporção do PIB), despesas em pesquisa e desenvolvimento (proporção do PIB), taxa de alfabetização (proporção de pessoas com 15 anos de idade ou mais), energia alternativa e nuclear (proporção do uso total de energia), área florestal (proporção da área da terra), exportações de combustível (proporção das exportações de mercadorias), despesas (proporção do PIB), terra agrícola (proporção da área terrestre), população rural (proporção da população total), população urbana (proporção da população total), saneamento (proporção da população com acesso), saneamento urbano (proporção da população urbana com acesso) e títulos da dívida externa (proporção do rendimento nacional bruto (RNB)). Estas são variáveis que se encaixam no nosso estudo e que expressam as formas e características que queremos estudar. As proporções/taxas são referentes a 195 países independentes listados no site da Nations Online. Dependendo da variável o ano de referência pode ser 2014, 2015 ou 2016. Todos os resultados a seguir foram obtidos utilizando o software R (R Core Team, 2017). Segue a descrição de cada variável.

Exportações de alta tecnologia: Produtos de alta tecnologia referem-se àqueles com alta intensidade de P\&D, como itens aeroespaciais, computadores, produtos farmacêuticos, instrumentos científicos e máquinas elétricas. A variável é a proporção do total de exportações de produtos de alta tecnologia sobre o total de exportações de produtos manufaturados.

Despesas em educação: Referem-se a despesas em educação pública incluindo gastos de governos municipais, regionais e nacionais em instituições educacionais, administração de educação e subsídios para entidades privadas. A variável é calculada dividindo a despesa pública total em educação incorrida por todas as agências / departamentos governamentais 
pela despesa total do governo.

Terra arável: As terras aráveis incluem terras definidas pela Organização das Nações Unidas para Alimentação e Agricultura como terras sob lavouras temporárias, prados temporários para corte ou para pastagem, etc. A variável é a proporção do território que corresponde a terras aráveis.

Serviços: Serviços correspondem a valores agregados no comércio, transporte e serviços governamentais, financeiros, profissionais e pessoais, tais como educação, cuidados de saúde e serviços imobiliários. Também estão incluídas as taxas imputadas ao serviço bancário e os direitos de importação. A variável é a proporção do PIB gasto em serviços.

Despesas em pesquisa e desenvolvimento: As despesas com pesquisa e desenvolvimento são gastos de capital realizados sistematicamente para aumentar o conhecimento, incluindo o conhecimento da humanidade, a cultura e a sociedade, e o uso do conhecimento para novas aplicações. A variável é a proporção do PIB gasto em pequisa e desenvolvimento.

Taxa de alfabetização: A taxa de alfabetização de adultos é a proporção de pessoas com mais de 15 anos de idade, que podem ler e escrever com compreensão.

Energia alternativa e nuclear: A energia alternativa e nuclear é a energia limpa e a energia não carboidratada, que não produz dióxido de carbono quando gerada. Inclui energia hidrelétrica e nuclear, geotérmica e solar, entre outras. A variável é calculada dividindo o consumo de energia alternativa e nuclear pelo consumo total de energia.

Área florestal: A área florestal é terra sob cerco natural ou plantação de árvores, seja produtiva ou não, e exclui as árvores em sistemas de produção agrícola e árvores em parques e jardins urbanos. A variável é a proporção do território que tem área florestal.

Exportações de combustível: Os combustíveis compreendem as commodities de combustíveis minerais, lubrificantes e materiais relacionados. A variável é calculada dividindo o total das exportações em combustível pelo total de exportações.

Despesas: Despesas referem-se pagamento em dinheiro para atividades operacionais do governo no fornecimento de bens e serviços. Inclui remuneração de funcionários, juros e subsídios, benefícios sociais e outras despesas, tais como aluguel e dividendos. A variável corresponde à proporção do PIB gasto em despesas.

Terra agrícola: Terras agrícolas são terras cultiváveis, sob culturas permanentes e em pastagens permanentes. Não precisa ser replantada após cada colheita, como é o caso de cacau, café e borracha. Esta categoria inclui terras sob arbustos floridos, árvores frutíferas, nozes e videiras. A variável é a proporção do território que corresponde a terras agrícolas.

População rural: A população rural refere-se a pessoas que vivem em áreas rurais, conforme definido pelos escritórios nacionais de estatística. É calculada como a diferença entre população total e população urbana. A variável corresponde à proporção da população total que vivem em áreas rurais. 
População urbana: A população urbana refere-se a pessoas que vivem em áreas urbanas, conforme definido pelos escritórios nacionais de estatística. A variável corresponde à proporção da população total que vivem em áreas urbanas.

Saneamento: O acesso a saneamento refere-se à proporção da população usando instalações de saneamento. As instalações de saneamento são susceptíveis de garantir a separação higiênica de excretas humanas do contato humano.

Saneamento urbano: O acesso a saneamento urbano refere-se à proporção da população urbana utilizando instalações de saneamento.

Títulos da dívida externa: A dívida externa é a soma das dívidas de longo prazo públicas, uso do crédito do FMI e dívida de curto prazo. A variável é calculada dividindo a soma de todas as dívidas externas pelo rendimento nacional, com truncamento em 1.

Para cada variável ajustamos os seguintes modelos: beta, Kumaraswamy, simplex, gama unitário, beta retangular, logito logístico, logito normal, logito t, logito exponencial potência, logito skew logístico, logito skew normal, logito skew t e logito skew exponencial potência. Calculamos as seguintes medidas de qualiadde de ajuste: AIC, BIC, $w_{\mathrm{AIC}}, w_{\mathrm{BIC}}$ e a estatística de teste $V_{\mathrm{RV}}$. Realizamos o teste da razão de verossimilhanças generalizado, sob a hipótese nula de que o modelo correto é beta, e reportamos o respectivo p-valor. Continuamos a análise com o modelo beta, considerado aqui como de referência, e dois modelos selecionados usando os diversos critérios. A seleção é feita com alguma subjetividade considerando conjuntamente $w_{\mathrm{AIC}}, w_{\mathrm{BIC}}$ e $p_{V}$.

Nas Tabelas ímpares de 4.1 a 4.31 são apresentados os AIC's, BIC's, wAIC's, w $w_{\text {BIC's e }}$ $p_{V}$ 's para cada modelo. O melhor modelo sob cada critério está sublinhado. A rejeição do modelo beta pelo teste TRVG a 10\% (5\%,1\%) será indicado com um asterisco (dois, três) junto ao valor de $p_{V}$. Nas Figuras 4.1 a 4.16 estão as fdp e fda estimadas do modelo beta e fdp's e fda's estimadas dos dois modelos selecionados, sempre com a distribuição beta com linha contínua. Em verde temos a densidade obtida por suavização e a fda empírica. Por fim, nas Tabelas pares de 4.2 a 4.32 apresentamos algumas medidas descritivas e estimativas para cada variável utilizando o modelo beta e os modelos selecionados. ${ }^{1}$

\footnotetext{
${ }^{1}$ Nestas tabelas dp significa desvio padrão.
} 
Tabela 4.1: Critérios de seleção de modelos, Exportações de alta tecnologia; $n=123$, ano $=2015$.

\begin{tabular}{l|rrrrrr}
\hline Dist & AIC & BIC & $w_{\text {AIC }}$ & $w_{\text {BIC }}$ & $V_{R V}$ & $p_{V}$ \\
\hline Beta & -338.006 & -332.382 & $\underline{0.356}$ & $\underline{0.470}$ & & \\
Kuma & -337.486 & -331.862 & 0.275 & 0.362 & -0.447 & 0.673 \\
Simplex & 89.703 & 95.328 & 0.000 & 0.000 & -4.177 & 1.000 \\
Gu & -334.539 & -328.915 & 0.063 & 0.083 & -1.012 & 0.844 \\
BR & -336.006 & -327.570 & 0.131 & 0.042 & 0.000 & 0.500 \\
LL & -319.476 & -313.852 & 0.000 & 0.000 & -2.543 & 0.995 \\
LN & -307.170 & -301.546 & 0.000 & 0.000 & -2.161 & 0.985 \\
LT & -322.692 & -312.256 & 0.000 & 0.000 & -2.536 & 0.994 \\
LEP & -320.829 & -312.392 & 0.000 & 0.000 & -2.244 & 0.988 \\
LSL & -335.815 & -327.379 & 0.119 & 0.038 & -0.223 & 0.588 \\
LSN & -305.170 & -296.733 & 0.000 & 0.000 & -2.157 & 0.984 \\
LST & -332.635 & -321.387 & 0.024 & 0.002 & -0.388 & 0.651 \\
LSEP & -333.151 & -321.903 & 0.031 & 0.002 & -0.212 & 0.584 \\
\hline$*=$ Rej. $10 \%, * *=$ Rej. $5 \% \mathrm{e}^{* * *}=$ Rej. $1 \%$. & & &
\end{tabular}

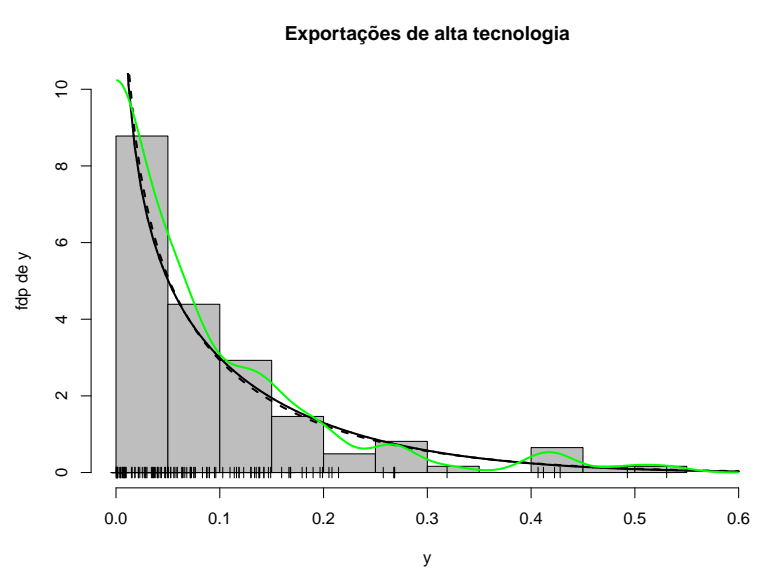

(a) fdp

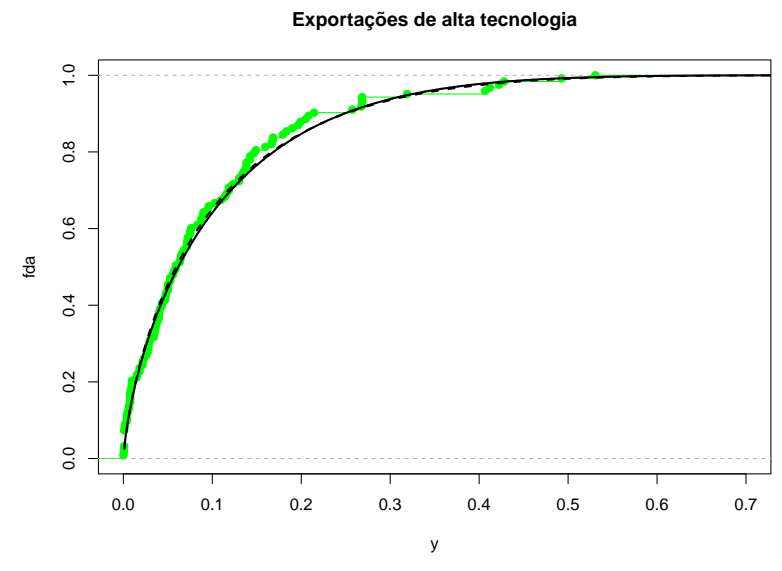

(b) fda

Figura 4.1: Fdp e fda ajustadas, beta (continua), Kumaraswamy (tracejada) e BR (pontilhada), suavização e fda empírica (verde); Exportações de alta tecnologia.

Tabela 4.2: Medidas descritivas e estimativas, Exportações de alta tecnologia.

\begin{tabular}{l|ccccccccc}
\hline Dist. & $y_{0.05}$ & $y_{0.10}$ & $y_{0.25}$ & $y_{0.50}$ & $y_{0.75}$ & $y_{0.90}$ & $y_{0.95}$ & média & $\mathrm{dp}$ \\
\hline Empírica & 0.001 & 0.004 & 0.022 & 0.059 & 0.136 & 0.213 & 0.314 & 0.097 & 0.108 \\
Beta & 0.003 & 0.006 & 0.020 & 0.062 & 0.143 & 0.249 & 0.323 & 0.097 & 0.107 \\
Kuma & 0.003 & 0.006 & 0.020 & 0.060 & 0.140 & 0.250 & 0.330 & 0.097 & 0.110 \\
BR & 0.003 & 0.006 & 0.020 & 0.062 & 0.143 & 0.249 & 0.323 & 0.097 & 0.107 \\
\hline
\end{tabular}


Tabela 4.3: Critérios de seleção de modelos, Despesas em educação; $n=83$, ano $=2014$.

\begin{tabular}{l|rrrrrr}
\hline Dist & AIC & BIC & $w_{\text {AIC }}$ & $w_{\text {BIC }}$ & $V_{R V}$ & $p_{V}$ \\
\hline Beta & -260.993 & -256.155 & $\underline{0.238}$ & $\underline{0.309}$ & & \\
Kuma & -259.600 & -254.763 & 0.119 & 0.154 & -0.363 & 0.642 \\
Simplex & -257.945 & -253.107 & 0.052 & 0.067 & -1.015 & 0.845 \\
Gu & -260.446 & -255.608 & 0.181 & 0.235 & -0.719 & 0.764 \\
BR & -258.993 & -251.736 & 0.088 & 0.034 & 0.000 & 0.500 \\
LL & -257.183 & -252.346 & 0.035 & 0.046 & -2.050 & 0.980 \\
LN & -258.719 & -253.881 & 0.076 & 0.099 & -1.017 & 0.845 \\
LT & -258.859 & -249.602 & 0.082 & 0.012 & -1.148 & 0.874 \\
LEP & -256.767 & -249.511 & 0.029 & 0.011 & -0.882 & 0.811 \\
LSL & -257.759 & -250.502 & 0.047 & 0.018 & -0.671 & 0.749 \\
LSN & -256.719 & -249.462 & 0.028 & 0.011 & -1.018 & 0.846 \\
LST & -255.453 & -245.778 & 0.015 & 0.002 & -1.224 & 0.889 \\
LSEP & -254.796 & -245.121 & 0.011 & 0.001 & -0.853 & 0.803 \\
\hline$*=$ Rej. $10 \%, * *=$ Rej. 5\% e *** = Rej. $1 \%$. & & &
\end{tabular}

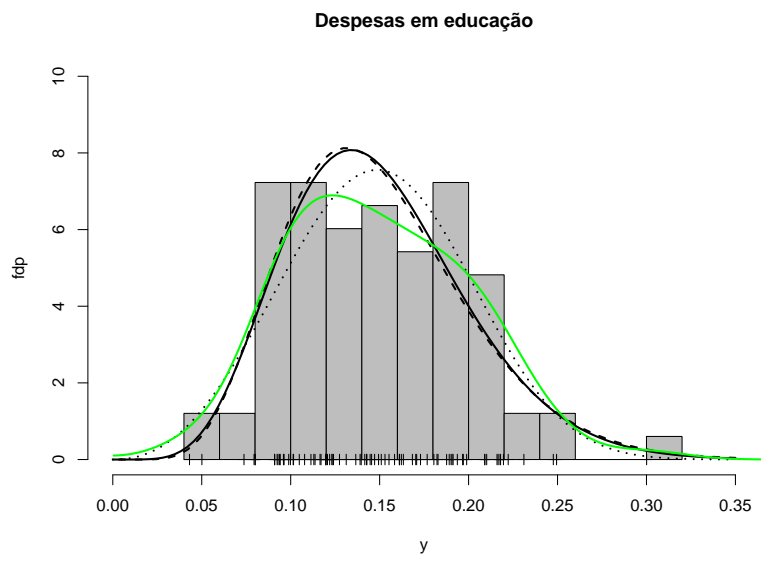

(a) fdp

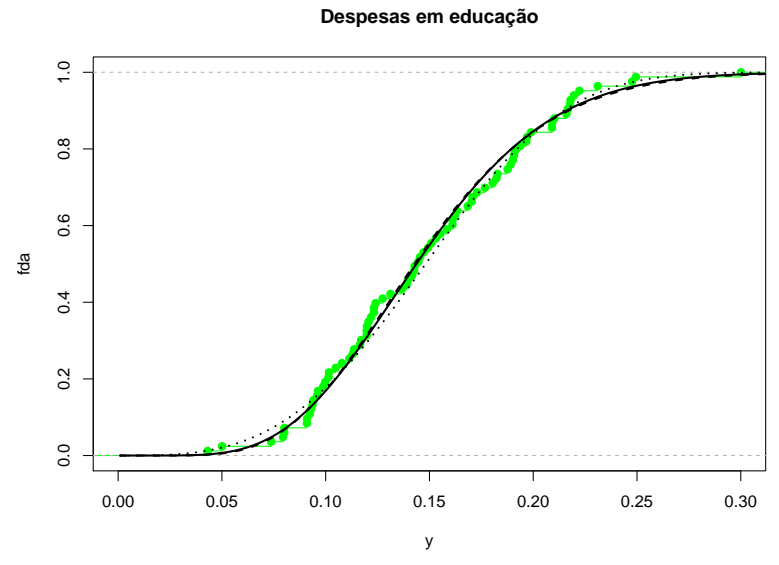

(b) fda

Figura 4.2: Fdp e fda ajustadas, beta (contínua), Gu (tracejada) e Kumaraswamy (pontilhada), suavização e fda empírica (verde); Despesas em educação.

Tabela 4.4: Medidas descritivas e estimativas, Despesas em educação.

\begin{tabular}{l|ccccccccc}
\hline Dist. & $y_{0.05}$ & $y_{0.10}$ & $y_{0.25}$ & $y_{0.50}$ & $y_{0.75}$ & $y_{0.90}$ & $y_{0.95}$ & média & $\mathrm{dp}$ \\
\hline Empírica & 0.080 & 0.092 & 0.112 & 0.145 & 0.188 & 0.216 & 0.222 & 0.149 & 0.050 \\
Beta & 0.075 & 0.088 & 0.112 & 0.144 & 0.180 & 0.216 & 0.239 & 0.149 & 0.050 \\
$\mathrm{Gu}$ & 0.076 & 0.088 & 0.112 & 0.143 & 0.180 & 0.218 & 0.242 & 0.149 & 0.051 \\
Kuma & 0.067 & 0.083 & 0.113 & 0.149 & 0.184 & 0.215 & 0.233 & 0.149 & 0.051 \\
\hline
\end{tabular}


Tabela 4.5: Critérios de seleção de modelos, Terra arável; $n=190$, ano $=2014$.

\begin{tabular}{l|rrrrrr}
\hline Dist & AIC & BIC & $w_{\text {AIC }}$ & $w_{\text {BIC }}$ & $V_{R V}$ & $p_{V}$ \\
\hline Beta & -348.223 & -341.728 & 0.284 & 0.330 & & \\
Kuma & -348.322 & $\underline{-341.828}$ & $\underline{0.299}$ & $\underline{0.347}$ & 0.289 & 0.386 \\
Simplex & -256.604 & -250.110 & 0.000 & 0.000 & -2.877 & 0.998 \\
Gu & -347.927 & -341.433 & 0.245 & 0.285 & -0.141 & 0.556 \\
BR & -346.223 & -336.481 & 0.105 & 0.024 & 0.000 & 0.500 \\
LL & -331.931 & -325.437 & 0.000 & 0.000 & -2.946 & 0.998 \\
LN & -332.794 & -326.300 & 0.000 & 0.000 & -2.017 & 0.978 \\
LT & -333.195 & -321.454 & 0.000 & 0.000 & -2.224 & 0.987 \\
LEP & -331.250 & -321.509 & 0.000 & 0.000 & -2.271 & 0.988 \\
LSL & -345.137 & -335.396 & 0.061 & 0.014 & -2.023 & 0.978 \\
LSN & -330.794 & -321.052 & 0.000 & 0.000 & -2.017 & 0.978 \\
LST & -339.394 & -326.406 & 0.003 & 0.000 & -1.651 & 0.951 \\
LSEP & -338.225 & -325.236 & 0.002 & 0.000 & -1.478 & 0.930 \\
\hline$*=$ Rej. $10 \%, * *=$ Rej. $5 \% \mathrm{e} * * *=$ Rej. $1 \%$. & & &
\end{tabular}

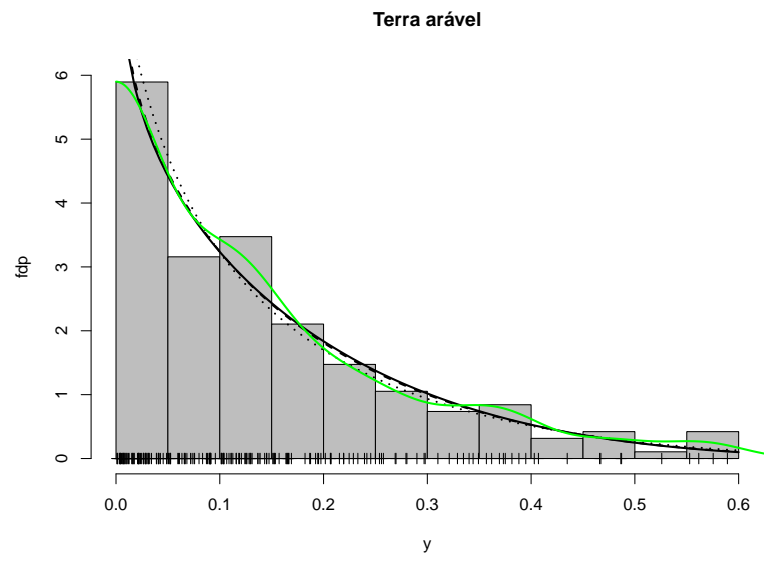

(a) fdp

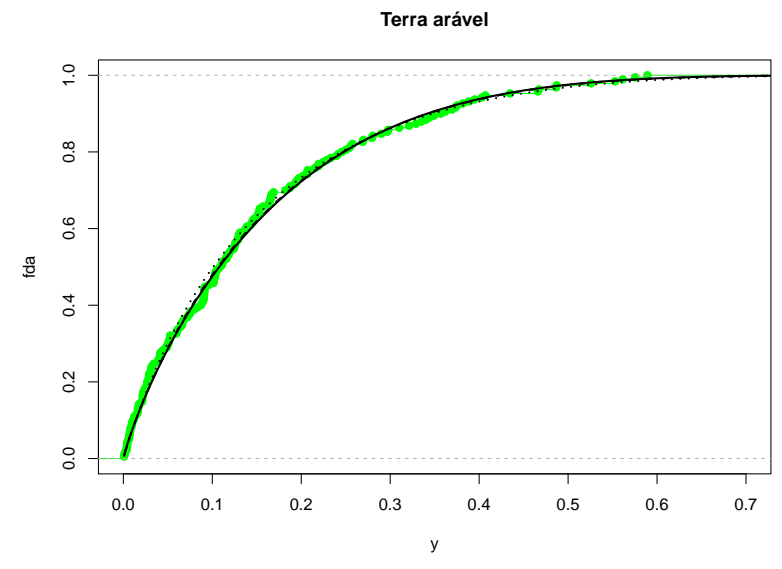

(b) fda

Figura 4.3: Fdp e fda ajustadas, beta (continua), Kumaraswamy (tracejada) e Gu (pontilhada), suavização e fda empírica (verde); Terra arável.

Tabela 4.6: Medidas descritivas e estimativas, Terra arável.

\begin{tabular}{l|ccccccccc}
\hline Dist. & $y_{0.05}$ & $y_{0.10}$ & $y_{0.25}$ & $y_{0.50}$ & $y_{0.75}$ & $y_{0.90}$ & $y_{0.95}$ & média & $\mathrm{dp}$ \\
\hline Empírica & 0.007 & 0.012 & 0.039 & 0.110 & 0.207 & 0.357 & 0.422 & 0.147 & 0.137 \\
Beta & 0.007 & 0.014 & 0.043 & 0.108 & 0.216 & 0.342 & 0.425 & 0.147 & 0.135 \\
Kuma & 0.007 & 0.014 & 0.042 & 0.107 & 0.215 & 0.343 & 0.427 & 0.147 & 0.136 \\
$\mathrm{Gu}$ & 0.007 & 0.015 & 0.040 & 0.101 & 0.211 & 0.349 & 0.442 & 0.146 & 0.141 \\
\hline
\end{tabular}


Tabela 4.7: Critérios de seleção de modelos, Serviços; $n=133$, ano $=2016$.

\begin{tabular}{l|rrrrrr}
\hline Dist & AIC & BIC & $w_{\text {AIC }}$ & $w_{\text {BIC }}$ & $V_{R V}$ & $p_{V}$ \\
\hline Beta & -190.239 & -184.459 & 0.135 & 0.203 & & \\
Kuma & -186.567 & -180.787 & 0.021 & 0.032 & -1.775 & 0.962 \\
Simplex & -190.714 & $\underline{-184.933}$ & $\underline{0.171}$ & $\underline{0.257}$ & 0.243 & 0.404 \\
Gu & -190.364 & -184.583 & 0.143 & 0.216 & 1.205 & 0.114 \\
BR & -188.239 & -179.568 & 0.050 & 0.018 & 0.000 & 0.500 \\
LL & -184.392 & -178.611 & 0.007 & 0.011 & -3.55 & 1.000 \\
LN & -190.058 & -184.278 & 0.123 & 0.185 & -0.189 & 0.575 \\
LT & -190.058 & -179.387 & 0.123 & 0.016 & -0.189 & 0.575 \\
LEP & -189.775 & -181.103 & 0.107 & 0.038 & 0.450 & 0.326 \\
LSL & -185.094 & -176.423 & 0.010 & 0.004 & -1.348 & 0.911 \\
LSN & -188.059 & -179.387 & 0.045 & 0.016 & -0.188 & 0.575 \\
LST & -186.075 & -174.514 & 0.017 & 0.001 & -0.173 & 0.569 \\
LSEP & -188.172 & -176.610 & 0.048 & 0.004 & 0.552 & 0.290 \\
\hline$*=$ Rej. $10 \%, * *=$ Rej. 5\% e *** = Rej. $1 \%$. & & &
\end{tabular}

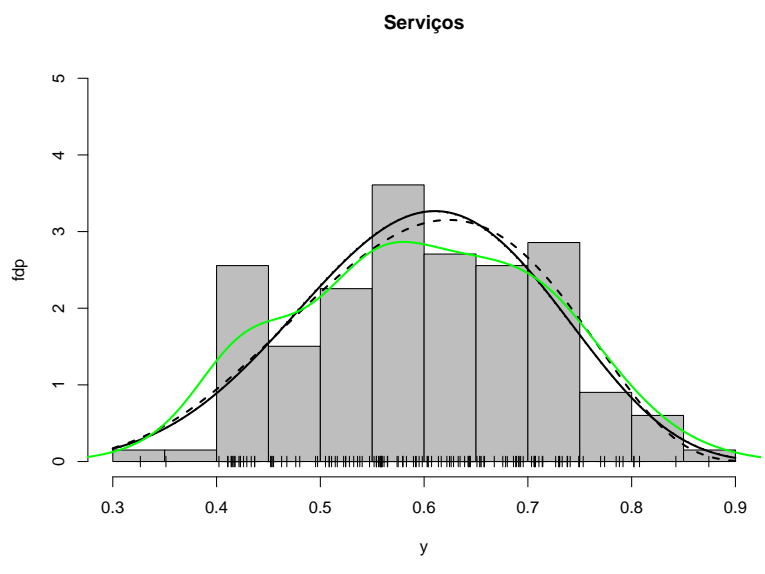

(a) fdp

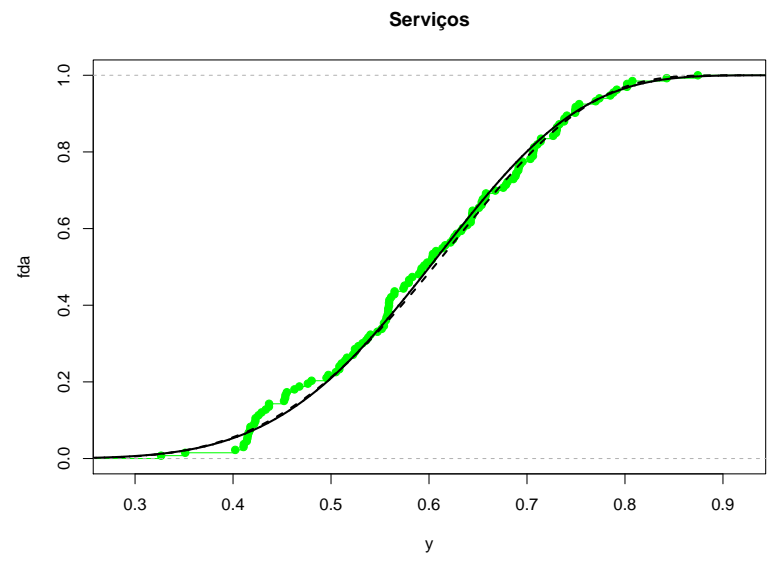

(b) fda

Figura 4.4: Fdp e fda ajustadas, beta (contínua), simplex (tracejada) e Gu (pontilhada), suavização e fda empírica (verde); Serviços.

Tabela 4.8: Medidas descritivas e estimativas, Serviços.

\begin{tabular}{l|ccccccccc}
\hline Dist. & $y_{0.05}$ & $y_{0.10}$ & $y_{0.25}$ & $y_{0.50}$ & $y_{0.75}$ & $y_{0.90}$ & $y_{0.95}$ & média & $\mathrm{dp}$ \\
\hline Empírica & 0.415 & 0.424 & 0.514 & 0.595 & 0.691 & 0.747 & 0.786 & 0.597 & 0.118 \\
Beta & 0.397 & 0.441 & 0.517 & 0.601 & 0.681 & 0.747 & 0.783 & 0.597 & 0.117 \\
Simplex & 0.394 & 0.438 & 0.517 & 0.605 & 0.686 & 0.749 & 0.782 & 0.599 & 0.105 \\
$\mathrm{Gu}$ & 0.398 & 0.442 & 0.517 & 0.601 & 0.681 & 0.747 & 0.784 & 0.597 & 0.117 \\
\hline
\end{tabular}


Tabela 4.9: Critérios de seleção de modelos, Despesas em pesquisa e desenvolvimento; $n=69$, ano $=2015$.

\begin{tabular}{l|rrrrrr}
\hline Dist & AIC & BIC & $w_{\text {AIC }}$ & $w_{\text {BIC }}$ & $V_{R V}$ & $p_{V}$ \\
\hline Beta & -476.275 & -471.807 & 0.166 & 0.249 & & \\
Kuma & -476.187 & -471.719 & 0.159 & 0.239 & -0.417 & 0.662 \\
Simplex & -468.857 & -464.389 & 0.004 & 0.006 & -0.900 & 0.816 \\
Gu & -476.532 & -472.064 & $\underline{0.189}$ & $\underline{0.284}$ & 0.110 & 0.456 \\
BR & -474.275 & -467.573 & 0.061 & 0.030 & 0.014 & 0.494 \\
LL & -469.724 & -465.256 & 0.006 & 0.009 & -1.833 & 0.967 \\
LN & -473.736 & -469.268 & 0.047 & 0.070 & -0.606 & 0.728 \\
LT & -473.736 & -465.034 & 0.047 & 0.008 & -0.606 & 0.728 \\
LEP & -475.457 & -468.755 & 0.111 & 0.054 & 0.182 & 0.428 \\
LSL & -472.982 & -466.280 & 0.032 & 0.016 & -0.430 & 0.666 \\
LSN & -471.736 & -465.033 & 0.017 & 0.008 & -0.606 & 0.728 \\
LST & -469.737 & -460.800 & 0.006 & 0.001 & -0.605 & 0.728 \\
LSEP & -476.110 & -467.173 & 0.153 & 0.025 & 0.664 & 0.253 \\
\hline$*=$ Rej. $10 \%, * *=$ Rej. $5 \% \mathrm{e}{ }^{* * *}=$ Rej. $1 \%$. & & &
\end{tabular}

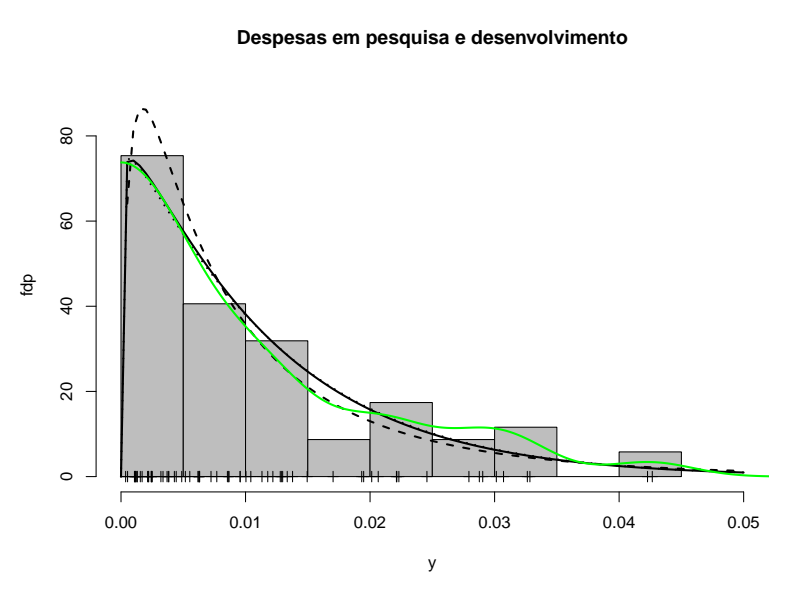

(a) fdp

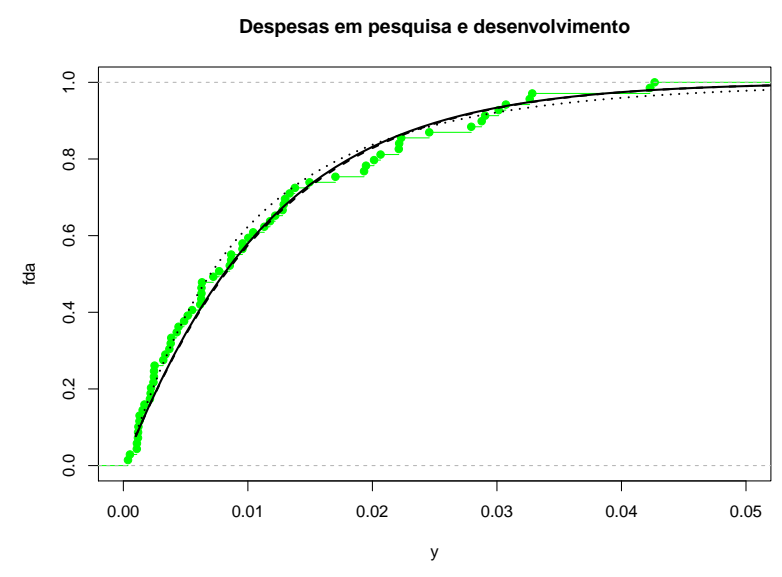

(b) fda

Figura 4.5: Fdp e fda ajustadas, beta (contínua), Gu (tracejada) e Kumaraswamy (pontilhada), suavização e fda empírica (verde); Despesas em pesquisa e desenvolvimento.

Tabela 4.10: Medidas descritivas e estimativas, Despesas em pesquisa e desenvolvimento.

\begin{tabular}{l|ccccccccc}
\hline Dist. & $y_{0.05}$ & $y_{0.10}$ & $y_{0.25}$ & $y_{0.50}$ & $y_{0.75}$ & $y_{0.90}$ & $y_{0.95}$ & média & $\mathrm{dp}$ \\
\hline Empírica & 0.001 & 0.001 & 0.003 & 0.008 & 0.017 & 0.029 & 0.032 & 0.011 & 0.011 \\
Beta & 0.001 & 0.002 & 0.004 & 0.009 & 0.016 & 0.026 & 0.033 & 0.011 & 0.011 \\
$\mathrm{Gu}$ & 0.001 & 0.002 & 0.003 & 0.007 & 0.015 & 0.027 & 0.037 & 0.012 & 0.013 \\
Kuma & 0.001 & 0.002 & 0.004 & 0.009 & 0.016 & 0.026 & 0.034 & 0.011 & 0.011 \\
\hline
\end{tabular}


Tabela 4.11: Critérios de seleção de modelos, Taxa de alfabetização; $n=36$, ano $=2014$.

\begin{tabular}{l|rrrrrr}
\hline Dist & AIC & BIC & $w_{\text {AIC }}$ & $w_{\text {BIC }}$ & $V_{R V}$ & $p_{V}$ \\
\hline Beta & -54.232 & -51.065 & 0.159 & 0.215 & & \\
Kuma & -54.145 & -50.978 & 0.153 & 0.206 & -0.587 & 0.721 \\
Simplex & -2.917 & 0.250 & 0.000 & 0.000 & -2.870 & 0.998 \\
Gu & -54.239 & $\underline{-51.071}$ & $\underline{0.160}$ & $\underline{0.215}$ & 0.374 & 0.354 \\
BR & -52.232 & -47.481 & 0.059 & 0.036 & 0.000 & 0.500 \\
LL & -53.519 & -50.352 & 0.112 & 0.150 & -0.201 & 0.580 \\
LN & -51.183 & -48.016 & 0.035 & 0.047 & -0.655 & 0.744 \\
LT & -53.778 & -47.027 & 0.127 & 0.029 & -0.137 & 0.555 \\
LEP & -52.084 & -47.333 & 0.054 & 0.033 & -0.034 & 0.514 \\
LSL & -52.815 & -48.064 & 0.079 & 0.048 & 0.363 & 0.358 \\
LSN & -49.183 & -44.433 & 0.013 & 0.008 & -0.657 & 0.744 \\
LST & -50.776 & -44.442 & 0.028 & 0.008 & 0.251 & 0.401 \\
LSEP & -50.244 & -43.909 & 0.022 & 0.006 & 0.003 & 0.499 \\
\hline
\end{tabular}

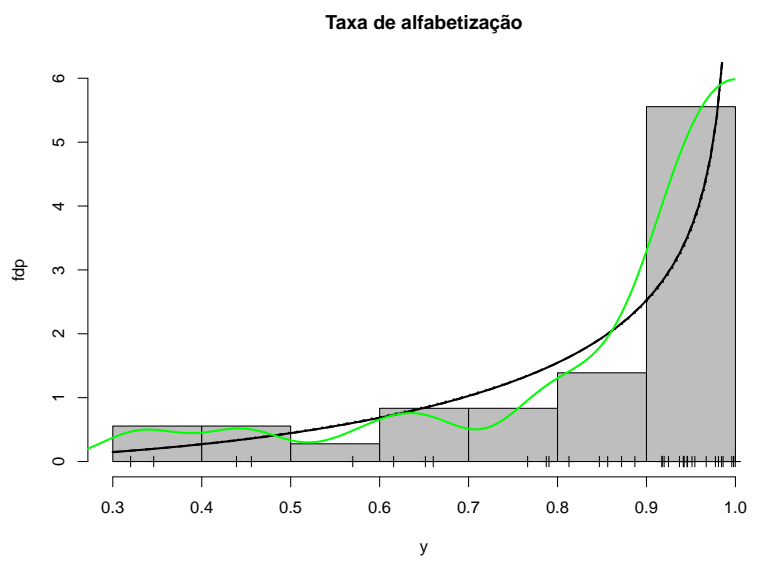

(a) fdp

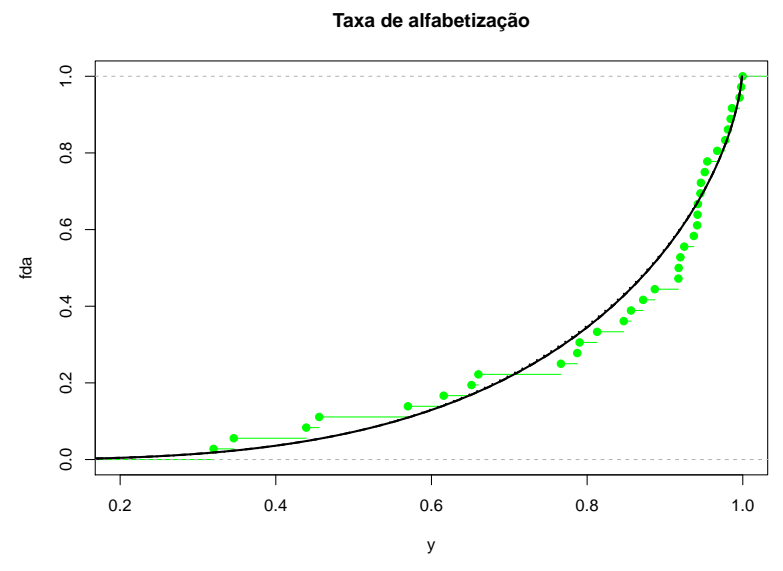

(b) fda

Figura 4.6: Fdp e fda ajustadas, beta (contínua), Gu (tracejada) e Kumaraswamy (pontilhada), suavização e fda empírica (verde); Taxa de alfabetização.

Tabela 4.12: Medidas descritivas e estimativas, Taxa de alfabetização.

\begin{tabular}{l|ccccccccc}
\hline Dist. & $y_{0.05}$ & $y_{0.10}$ & $y_{0.25}$ & $y_{0.50}$ & $y_{0.75}$ & $y_{0.90}$ & $y_{0.95}$ & média & $\mathrm{dp}$ \\
\hline Empírica & 0.416 & 0.513 & 0.782 & 0.919 & 0.952 & 0.985 & 0.996 & 0.828 & 0.193 \\
Beta & 0.445 & 0.555 & 0.731 & 0.882 & 0.963 & 0.990 & 0.996 & 0.825 & 0.179 \\
Gu & 0.445 & 0.555 & 0.732 & 0.882 & 0.963 & 0.990 & 0.996 & 0.825 & 0.179 \\
Kuma & 0.445 & 0.552 & 0.728 & 0.880 & 0.962 & 0.990 & 0.996 & 0.824 & 0.179 \\
\hline
\end{tabular}


Tabela 4.13: Critérios de seleção de modelos, Energia alternativa e nuclear; $n=133$, ano $=2015$.

\begin{tabular}{|c|c|c|c|c|c|c|}
\hline Dist & $\overline{\mathrm{AIC}}$ & $\overline{B I C}$ & $w_{\mathrm{AIC}}$ & $w_{\mathrm{BIC}}$ & $V_{R V}$ & $p_{V}$ \\
\hline Beta & -413.913 & -408.132 & 0.009 & 0.025 & & \\
\hline Kuma & -417.958 & -412.178 & 0.068 & 0.191 & 1.591 & $\underline{0.056}^{*}$ \\
\hline Simplex & 190.513 & 196.294 & 0.000 & 0.000 & $-\overline{3.438}$ & $\overline{1.000}$ \\
\hline $\mathrm{Gu}$ & -418.968 & -413.187 & 0.113 & 0.317 & 1.309 & $0.095^{*}$ \\
\hline $\mathrm{BR}$ & -422.031 & -413.360 & $\underline{0.521}$ & $\underline{0.346}$ & 1.411 & $0.079^{*}$ \\
\hline LL & $\overline{-409.525}$ & $\overline{-403.744}$ & $\overline{0.001}$ & $\overline{0.003}$ & -0.339 & 0.633 \\
\hline $\mathrm{LN}$ & -401.961 & -396.180 & 0.000 & 0.000 & -0.731 & 0.768 \\
\hline $\mathrm{LT}$ & -410.230 & -399.559 & 0.001 & 0.000 & -0.296 & 0.616 \\
\hline LEP & -406.151 & -397.480 & 0.000 & 0.000 & -0.432 & 0.667 \\
\hline LSL & -419.428 & -410.757 & 0.142 & 0.094 & 1.283 & $0.100^{*}$ \\
\hline LSN & -399.959 & -391.288 & 0.000 & 0.000 & -0.731 & 0.768 \\
\hline LST & -415.717 & -404.156 & 0.022 & 0.003 & 0.696 & 0.243 \\
\hline LSEP & -419.145 & -407.583 & 0.123 & 0.019 & 1.164 & 0.122 \\
\hline
\end{tabular}

${ }^{*}=$ Rej. $10 \%,{ }^{* *}=$ Rej. $5 \% \mathrm{e}^{* * *}=$ Rej. $1 \%$.

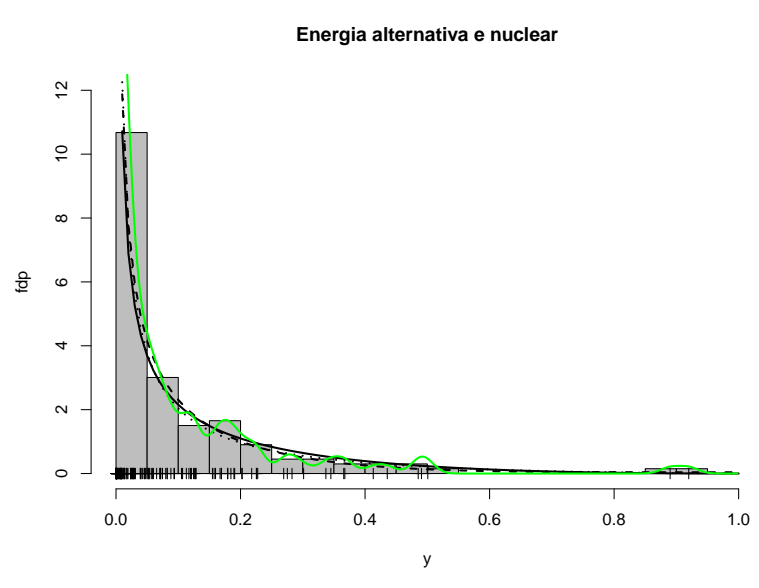

(a) fdp

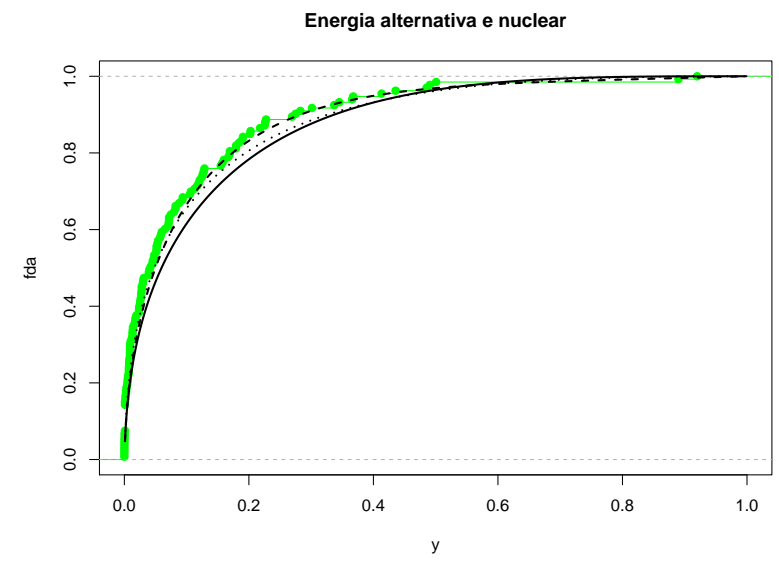

(b) fda

Figura 4.7: Fdp e fda ajustadas, beta (contínua), BR (tracejada) e Gu (pontilhada), suavização e fda empírica (verde); Energia alternativa e nuclear.

Tabela 4.14: Medidas descritivas e estimativas, Energia alternativa e nuclear.

\begin{tabular}{l|ccccccccc}
\hline Dist. & $y_{0.05}$ & $y_{0.10}$ & $y_{0.25}$ & $y_{0.50}$ & $y_{0.75}$ & $y_{0.90}$ & $y_{0.95}$ & média & $\mathrm{dp}$ \\
\hline Empírica & 0.000 & 0.001 & 0.008 & 0.042 & 0.128 & 0.274 & 0.386 & 0.101 & 0.153 \\
Beta & 0.002 & 0.003 & 0.014 & 0.060 & 0.175 & 0.338 & 0.450 & 0.111 & 0.147 \\
$\mathrm{BR}$ & 0.002 & 0.003 & 0.013 & 0.050 & 0.141 & 0.283 & 0.404 & 0.100 & 0.165 \\
$\mathrm{Gu}$ & 0.001 & 0.003 & 0.011 & 0.049 & 0.154 & 0.327 & 0.454 & 0.105 & 0.150 \\
\hline
\end{tabular}


Tabela 4.15: Critérios de seleção de modelos, Área florestal; $n=192$, ano $=2015$.

\begin{tabular}{l|rrrrrr}
\hline Dist & AIC & BIC & $w_{\text {AIC }}$ & $w_{\text {BIC }}$ & $V_{R V}$ & $p_{V}$ \\
\hline Beta & -89.548 & -83.033 & 0.031 & $\underline{0.269}$ & & \\
Kuma & -88.619 & -82.104 & 0.019 & 0.169 & -2.261 & 0.988 \\
Simplex & 278.336 & 284.851 & 0.000 & 0.000 & -3.906 & 1.000 \\
Gu & -86.823 & -80.308 & 0.008 & 0.069 & -2.387 & 0.992 \\
BR & -87.548 & -77.776 & 0.011 & 0.019 & 0.000 & 0.500 \\
LL & -68.160 & -61.645 & 0.000 & 0.000 & -3.492 & 1.000 \\
LN & -49.972 & -43.457 & 0.000 & 0.000 & -4.018 & 1.000 \\
LT & -76.081 & -64.308 & 0.000 & 0.000 & -1.860 & 0.969 \\
LEP & -79.180 & -69.408 & 0.000 & 0.000 & -0.783 & 0.783 \\
LSL & -92.220 & -82.448 & 0.117 & 0.201 & 0.945 & 0.172 \\
LSN & -47.968 & -38.195 & 0.000 & 0.000 & -4.017 & 1.000 \\
LST & -93.295 & -80.266 & 0.201 & 0.067 & 1.191 & 0.117 \\
LSEP & -95.520 & -82.490 & $\underline{0.612}$ & 0.205 & 1.261 & 0.104 \\
\hline
\end{tabular}

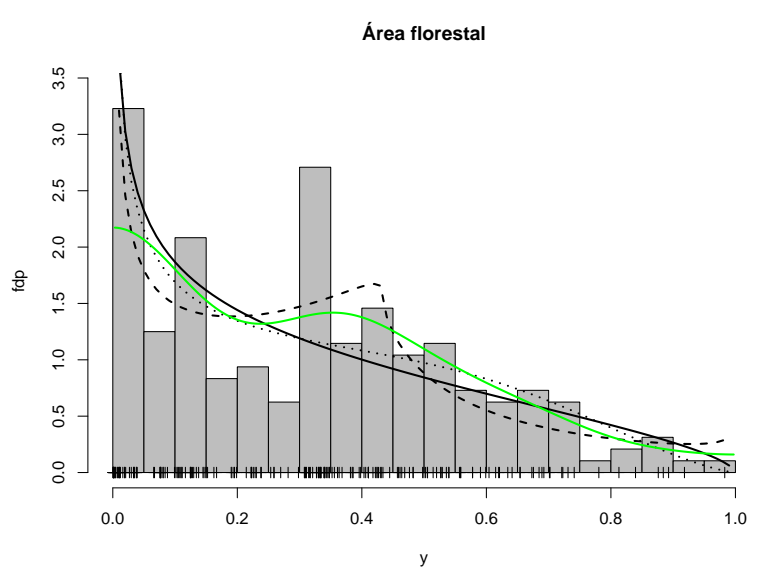

(a) fdp

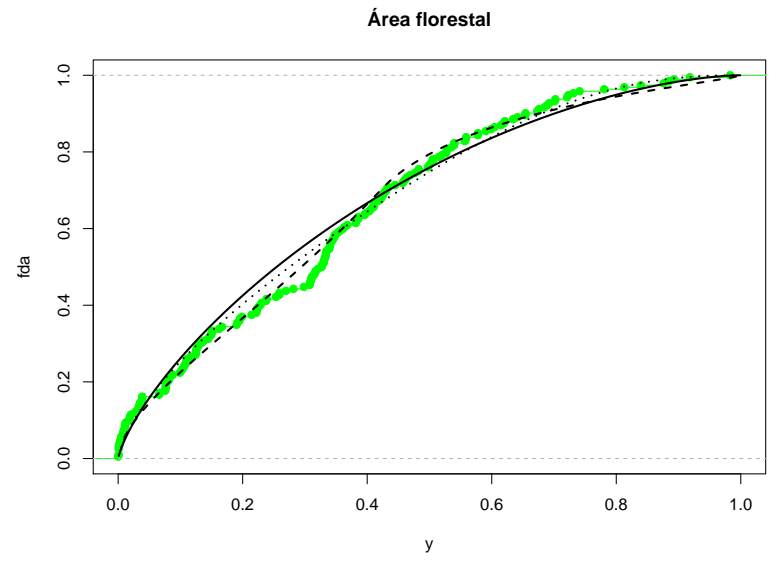

(b) fda

Figura 4.8: Fdp e fda ajustadas, beta (continua), LSEP (tracejada) e LSL (pontilhada), suavização e fda empírica (verde); Área florestal.

Tabela 4.16: Medidas descritivas e estimativas, Área florestal.

\begin{tabular}{l|ccccccccc}
\hline Dist. & $y_{0.05}$ & $y_{0.10}$ & $y_{0.25}$ & $y_{0.50}$ & $y_{0.75}$ & $y_{0.90}$ & $y_{0.95}$ & média & $\mathrm{dp}$ \\
\hline Empírica & 0.005 & 0.019 & 0.110 & 0.327 & 0.482 & 0.654 & 0.727 & 0.322 & 0.238 \\
Beta & 0.011 & 0.028 & 0.094 & 0.255 & 0.490 & 0.701 & 0.802 & 0.311 & 0.253 \\
LSEP & 0.008 & 0.027 & 0.117 & 0.294 & 0.456 & 0.675 & 0.819 & 0.323 & 0.243 \\
LSL & 0.010 & 0.026 & 0.099 & 0.276 & 0.503 & 0.680 & 0.765 & 0.318 & 0.245 \\
\hline
\end{tabular}


Tabela 4.17: Critérios de seleção de modelos, Exportações de combustivel; $n=125$, ano $=2015$.

\begin{tabular}{l|rrrrrl}
\hline Dist & AIC & BIC & $w_{\text {AIC }}$ & $w_{\text {BIC }}$ & $V_{R V}$ & $p_{V}$ \\
\hline Beta & -397.271 & -391.614 & 0.000 & 0.001 & & \\
Kuma & -398.986 & -393.329 & 0.000 & 0.003 & 0.895 & 0.185 \\
Simplex & 619.125 & 624.782 & 0.000 & 0.000 & -4.958 & 1.000 \\
Gu & -399.370 & -393.713 & 0.000 & 0.003 & 0.939 & 0.174 \\
BR & -411.442 & $\underline{-402.957}$ & 0.152 & $\underline{0.342}$ & $\underline{2.315}$ & $\underline{0.010^{* * *}}$ \\
LL & -404.050 & -398.394 & 0.004 & 0.035 & 0.556 & 0.289 \\
LN & -389.645 & -383.988 & 0.000 & 0.000 & -0.531 & 0.702 \\
LT & -409.728 & -399.243 & 0.065 & 0.053 & 0.998 & 0.159 \\
LEP & -409.860 & -401.375 & 0.069 & 0.155 & 1.121 & 0.131 \\
LSL & -406.144 & -397.659 & 0.011 & 0.024 & 1.682 & $0.046^{* *}$ \\
LSN & -387.644 & -379.159 & 0.000 & 0.000 & -0.530 & 0.702 \\
LST & -410.421 & -399.108 & 0.091 & 0.050 & 1.747 & $0.040^{* *}$ \\
LSEP & -414.213 & -402.900 & $\underline{0.608}$ & 0.333 & 1.775 & $0.038^{* *}$ \\
\hline
\end{tabular}

${ }^{*}=$ Rej. $10 \%,{ }^{* *}=$ Rej. $5 \% \mathrm{e}^{* * *}=$ Rej. $1 \%$.

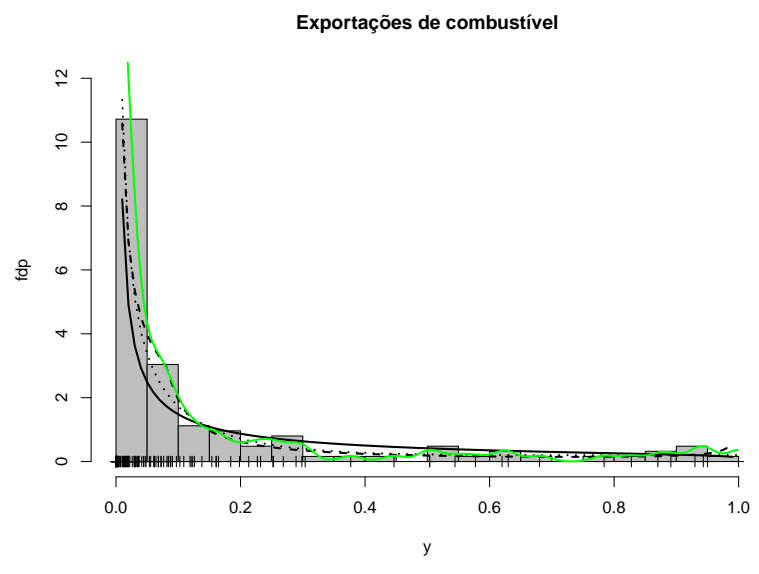

(a) fdp

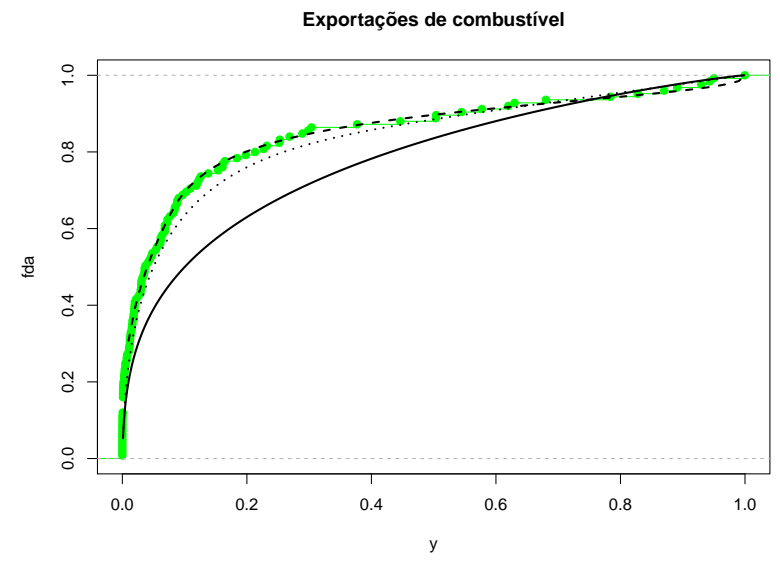

(b) fda

Figura 4.9: Fdp e fda ajustadas, beta (contínua), LSEP (tracejada) e BR (pontilhada), suavização e fda empírica (verde); Exportaçôes de combustivel.

Tabela 4.18: Medidas descritivas e estimativas, Exportações de combustível.

\begin{tabular}{l|ccccccccc}
\hline Dist. & $y_{0.05}$ & $y_{0.10}$ & $y_{0.25}$ & $y_{0.50}$ & $y_{0.75}$ & $y_{0.90}$ & $y_{0.95}$ & média & $\mathrm{dp}$ \\
\hline Empírica & 0.000 & 0.000 & 0.007 & 0.037 & 0.154 & 0.528 & 0.819 & 0.148 & 0.246 \\
Beta & 0.001 & 0.003 & 0.017 & 0.101 & 0.349 & 0.650 & 0.797 & 0.186 & 0.251 \\
LSEP & 0.000 & 0.000 & 0.005 & 0.040 & 0.137 & 0.517 & 0.839 & 0.149 & 0.250 \\
BR & 0.001 & 0.003 & 0.011 & 0.051 & 0.189 & 0.562 & 0.779 & 0.143 & 0.265 \\
\hline
\end{tabular}


Tabela 4.19: Critérios de seleção de modelos, Despesas; $n=94$, ano $=2015$.

\begin{tabular}{l|rrrrrl}
\hline Dist & AIC & BIC & $w_{\text {AIC }}$ & $w_{\text {BIC }}$ & $V_{R V}$ & $p_{V}$ \\
\hline Beta & -119.424 & -114.337 & 0.007 & 0.021 & & \\
Kuma & -115.570 & -110.483 & 0.001 & 0.003 & -1.29 & 0.901 \\
Simplex & -103.900 & -98.814 & 0.000 & 0.000 & -1.664 & 0.952 \\
Gu & -119.840 & -114.753 & 0.009 & 0.025 & 0.297 & 0.383 \\
BR & -126.106 & -118.476 & 0.208 & 0.164 & 1.173 & 0.120 \\
LL & -126.033 & -120.946 & 0.201 & $\underline{0.563}$ & 1.249 & 0.106 \\
LN & -117.408 & -112.321 & 0.003 & 0.008 & -0.419 & 0.662 \\
LT & -127.365 & -117.735 & $\underline{0.391}$ & 0.113 & 1.263 & 0.103 \\
LEP & -122.558 & -114.928 & 0.035 & 0.028 & 0.806 & 0.210 \\
LSL & -124.081 & -116.451 & 0.076 & 0.060 & 1.336 & $0.091^{*}$ \\
LSN & -115.408 & -107.778 & 0.001 & 0.001 & -0.419 & 0.662 \\
LST & -123.424 & -113.250 & 0.054 & 0.012 & 1.327 & $0.092^{*}$ \\
LSEP & -120.564 & -110.391 & 0.013 & 0.003 & 0.795 & 0.213 \\
\hline
\end{tabular}

$*=$ Rej. $10 \%,{ }^{* *}=$ Rej. $5 \%$ e ${ }^{* * *}=$ Rej. $1 \%$.

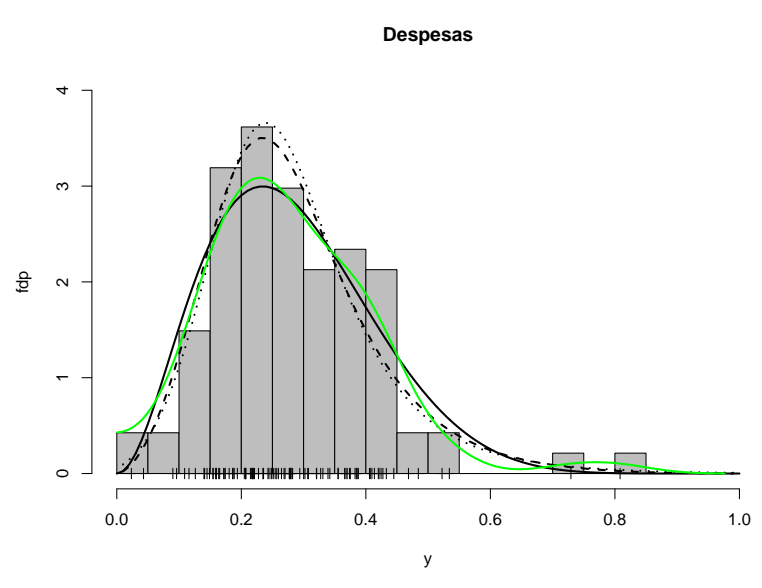

(a) fdp

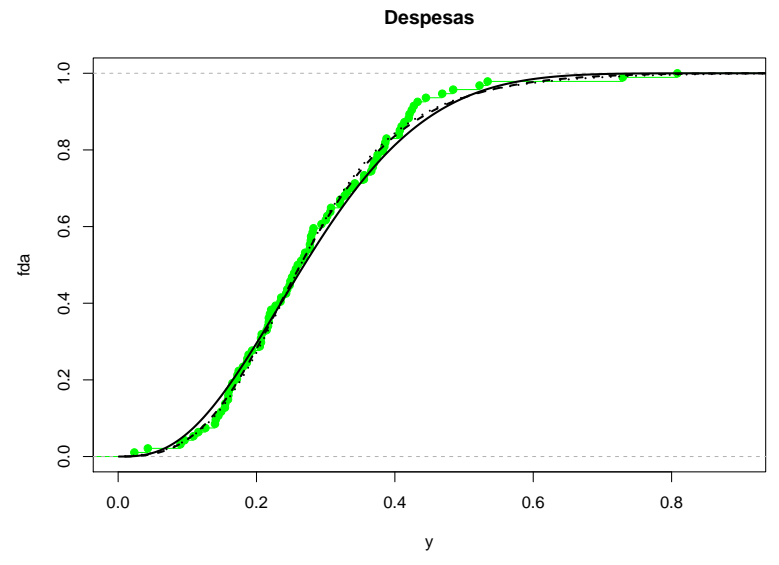

(b) fda

Figura 4.10: Fdp e fda ajustadas, beta (contínua), LL (tracejada) e LT (pontilhada), suavização e fda empirica (verde); Despesas.

Tabela 4.20: Medidas descritivas e estimativas, Despesas.

\begin{tabular}{l|ccccccccc}
\hline Dist. & $y_{0.05}$ & $y_{0.10}$ & $y_{0.25}$ & $y_{0.50}$ & $y_{0.75}$ & $y_{0.90}$ & $y_{0.95}$ & média & $\mathrm{dp}$ \\
\hline Empírica & 0.114 & 0.146 & 0.187 & 0.262 & 0.368 & 0.423 & 0.474 & 0.281 & 0.130 \\
Beta & 0.093 & 0.123 & 0.185 & 0.270 & 0.368 & 0.463 & 0.520 & 0.283 & 0.131 \\
LL & 0.104 & 0.134 & 0.190 & 0.263 & 0.352 & 0.453 & 0.524 & 0.282 & 0.130 \\
LT & 0.105 & 0.137 & 0.194 & 0.264 & 0.349 & 0.448 & 0.523 & 0.284 & 0.132 \\
\hline
\end{tabular}


Tabela 4.21: Critérios de seleção de modelos, Terra agrícola; $n=192$, ano $=2014$.

\begin{tabular}{l|rrllll}
\hline Dist & AIC & BIC & $w_{\text {AIC }}$ & $w_{\text {BIC }}$ & $V_{R V}$ & $p_{V}$ \\
\hline Beta & -59.087 & -52.572 & 0.022 & 0.087 & & \\
Kuma & -60.511 & -53.996 & 0.044 & 0.177 & $\underline{2.322}$ & $\underline{0.010^{* * *}}$ \\
Simplex & 2.658 & 9.173 & 0.000 & 0.000 & -2.632 & 0.996 \\
Gu & -56.999 & -50.484 & 0.008 & 0.031 & -3.141 & 0.999 \\
BR & -57.087 & -47.315 & 0.008 & 0.006 & 0.000 & 0.500 \\
LL & -50.344 & -43.829 & 0.000 & 0.001 & -4.793 & 1.000 \\
LN & -46.901 & -40.386 & 0.000 & 0.000 & -2.482 & 0.993 \\
LT & -50.424 & -38.651 & 0.000 & 0.000 & -4.555 & 1.000 \\
LEP & -47.859 & -38.087 & 0.000 & 0.000 & -4.275 & 1.000 \\
LSL & -66.484 & -56.712 & $\underline{0.873}$ & $\underline{0.690}$ & 1.659 & $0.049^{* *}$ \\
LSN & -44.899 & -35.127 & 0.000 & 0.000 & -2.486 & 0.994 \\
LST & -60.302 & -47.272 & 0.040 & 0.006 & 1.520 & $0.064^{*}$ \\
LSEP & -56.364 & -43.334 & 0.006 & 0.001 & 0.422 & 0.337 \\
\hline
\end{tabular}

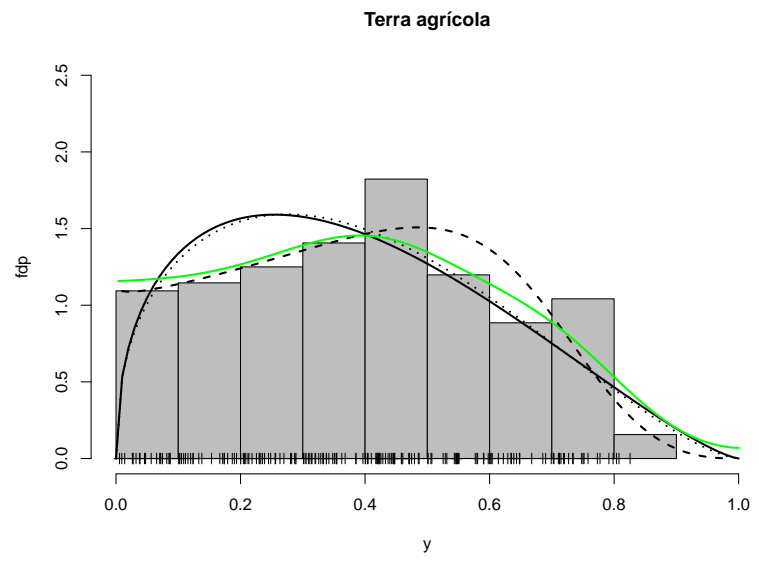

(a) fdp

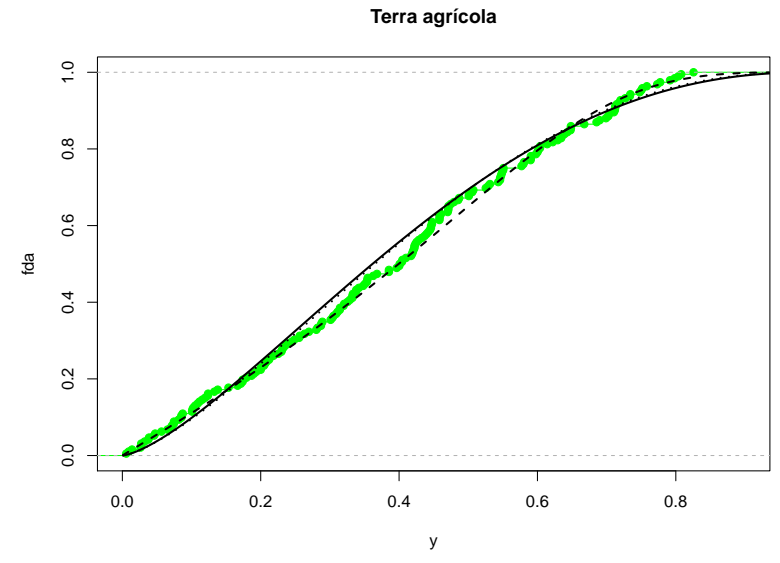

(b) fda

Figura 4.11: Fdp e fda ajustadas, beta (continua), LSL (tracejada) e Kumarwamy (pontilhada), suavização e fda empírica (verde); Terra agrícola.

Tabela 4.22: Medidas descritivas e estimativas, Terra agrícola.

\begin{tabular}{l|ccccccccc}
\hline Dist. & $y_{0.05}$ & $y_{0.10}$ & $y_{0.25}$ & $y_{0.50}$ & $y_{0.75}$ & $y_{0.90}$ & $y_{0.95}$ & média & $\mathrm{dp}$ \\
\hline Empírica & 0.047 & 0.086 & 0.213 & 0.402 & 0.557 & 0.712 & 0.749 & 0.393 & 0.220 \\
Beta & 0.062 & 0.102 & 0.203 & 0.362 & 0.546 & 0.703 & 0.782 & 0.384 & 0.222 \\
LSL & 0.046 & 0.091 & 0.217 & 0.400 & 0.568 & 0.687 & 0.746 & 0.396 & 0.218 \\
Kuma & 0.063 & 0.104 & 0.207 & 0.366 & 0.546 & 0.698 & 0.775 & 0.385 & 0.220 \\
\hline
\end{tabular}


Tabela 4.23: Critérios de seleção de modelos, População rural; $n=193$, ano $=2016$.

\begin{tabular}{l|rrrrrl}
\hline Dist & AIC & BIC & $w_{\text {AIC }}$ & $w_{\text {BIC }}$ & $V_{R V}$ & $p_{V}$ \\
\hline Beta & -33.429 & -26.904 & 0.016 & 0.090 & & \\
Kuma & -34.485 & -27.960 & 0.026 & 0.152 & $\underline{2.130}$ & $\underline{0.017^{* *}}$ \\
Simplex & 229.569 & 236.094 & 0.000 & 0.000 & -5.623 & 1.000 \\
Gu & -30.591 & -24.065 & 0.004 & 0.022 & -3.022 & 0.999 \\
BR & -31.429 & -21.641 & 0.006 & 0.006 & 0.000 & 0.500 \\
LL & -28.492 & -21.966 & 0.001 & 0.008 & -2.104 & 0.982 \\
LN & -3.389 & 3.136 & 0.000 & 0.000 & -2.683 & 0.996 \\
LT & -33.766 & -21.978 & 0.018 & 0.008 & 0.081 & 0.468 \\
LEP & -23.813 & -14.025 & 0.000 & 0.000 & -1.979 & 0.976 \\
LSL & -40.601 & -30.813 & $\underline{0.562}$ & $\underline{0.634}$ & 1.484 & $0.069^{*}$ \\
LSN & -1.388 & 8.400 & 0.000 & 0.000 & -2.672 & 0.996 \\
LST & -39.745 & -26.694 & 0.366 & 0.081 & 1.569 & $0.058^{*}$ \\
LSEP & -25.110 & -12.060 & 0.000 & 0.000 & -1.023 & 0.847 \\
* Rej. $10 \%, * *=$ Rej. 5\% e ${ }^{* * *}=$ Rej. $1 \%$.
\end{tabular}

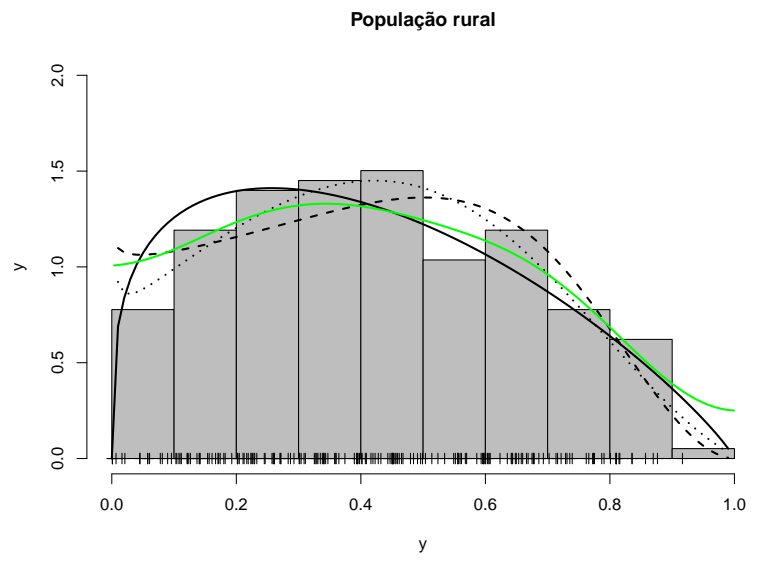

(a) fdp

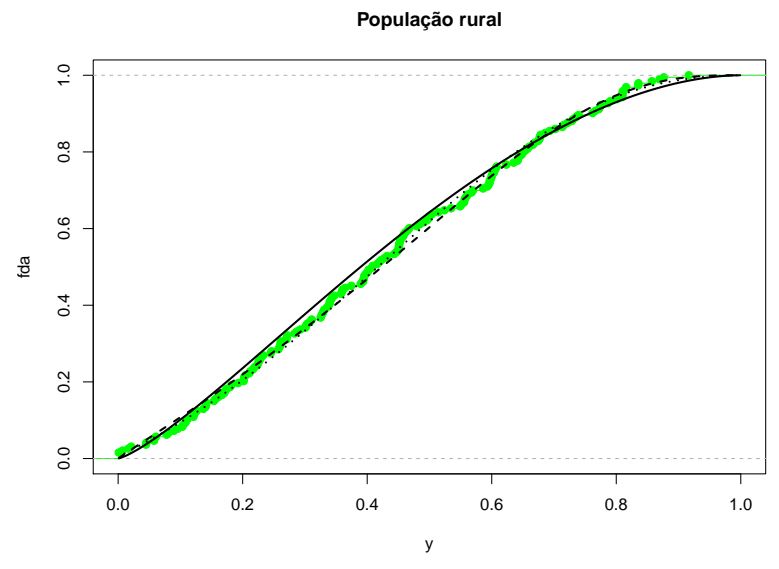

(b) fda

Figura 4.12: Fdp e fda ajustadas, beta (contínua), LSL (tracejada) e LST (pontilhada), suavização e fda empírica (verde); População rural.

Tabela 4.24: Medidas descritivas e estimativas, População rural.

\begin{tabular}{lccccccccc}
\hline Dist. & $y_{0.05}$ & $y_{0.10}$ & $y_{0.25}$ & $y_{0.50}$ & $y_{0.75}$ & $y_{0.90}$ & $y_{0.95}$ & média & $\mathrm{dp}$ \\
\hline Empírica & 0.060 & 0.114 & 0.226 & 0.409 & 0.604 & 0.758 & 0.810 & 0.422 & 0.234 \\
Beta & 0.057 & 0.099 & 0.211 & 0.390 & 0.594 & 0.757 & 0.834 & 0.411 & 0.241 \\
LSL & 0.047 & 0.093 & 0.227 & 0.424 & 0.610 & 0.741 & 0.804 & 0.422 & 0.236 \\
LST & 0.053 & 0.106 & 0.237 & 0.417 & 0.597 & 0.739 & 0.810 & 0.423 & 0.231 \\
\hline
\end{tabular}


Tabela 4.25: Critérios de seleção de modelos, População urbana; $n=193$, ano $=2016$.

\begin{tabular}{l|rrrrrl}
\hline Dist & AIC & BIC & $w_{\text {AIC }}$ & $w_{\text {BIC }}$ & $V_{R V}$ & $p_{V}$ \\
\hline Beta & -33.429 & -26.904 & 0.015 & 0.089 & & \\
Kuma & -32.835 & -26.310 & 0.011 & 0.066 & -1.911 & 0.972 \\
Simplex & 229.569 & 236.094 & 0.000 & 0.000 & -5.623 & 1.000 \\
Gu & -33.778 & -27.252 & 0.018 & 0.106 & $\underline{2.556}$ & $\underline{0.005}^{* * *}$ \\
BR & -31.429 & -21.641 & 0.006 & 0.006 & 0.000 & 0.500 \\
LL & -28.492 & -21.966 & 0.001 & 0.008 & -2.104 & 0.982 \\
LN & -3.389 & 3.136 & 0.000 & 0.000 & -2.683 & 0.996 \\
LT & -33.766 & -21.978 & 0.018 & 0.008 & 0.081 & 0.468 \\
LEP & -23.814 & -14.026 & 0.000 & 0.000 & -1.975 & 0.976 \\
LSL & -40.601 & -30.813 & $\underline{0.550}$ & $\underline{0.631}$ & 1.484 & $0.069^{*}$ \\
LSN & -1.389 & 8.399 & 0.000 & 0.000 & -2.671 & 0.996 \\
LST & -39.860 & -26.809 & 0.380 & 0.085 & 1.581 & $0.057^{*}$ \\
LSEP & -25.184 & -12.133 & 0.000 & 0.000 & -1.010 & 0.844 \\
\hline
\end{tabular}

${ }^{*}=$ Rej. $10 \%,{ }^{* *}=$ Rej. $5 \% \mathrm{e}^{* * *}=$ Rej. $1 \%$.

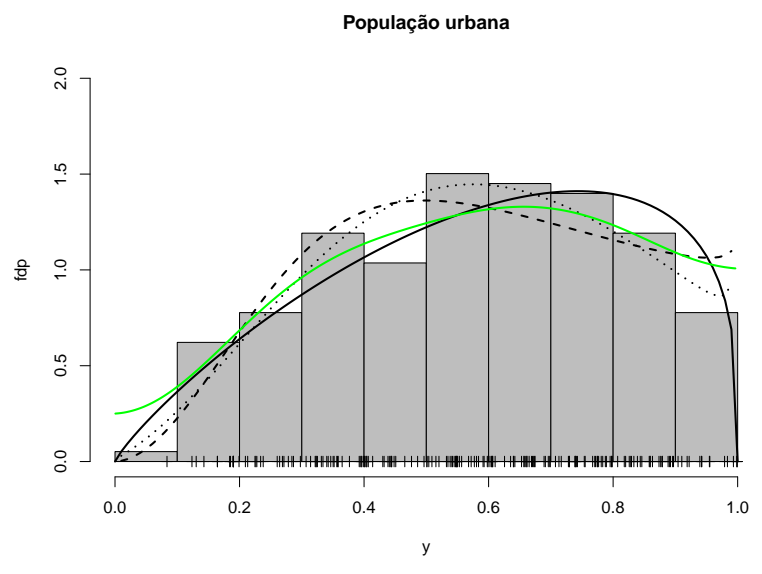

(a) fdp

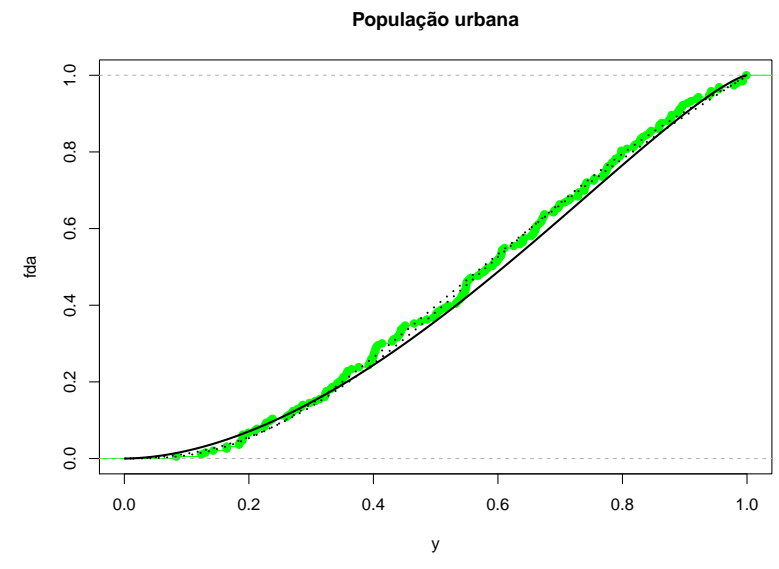

(b) fda

Figura 4.13: Fdp e fda ajustadas, beta (continua), LSL (tracejada) e LST (pontilhada), suavização e fda empírica (verde); População urbana.

Tabela 4.26: Medidas descritivas e estimativas, População urbana.

\begin{tabular}{l|ccccccccc}
\hline Dist. & $y_{0.05}$ & $y_{0.10}$ & $y_{0.25}$ & $y_{0.50}$ & $y_{0.75}$ & $y_{0.90}$ & $y_{0.95}$ & média & $\mathrm{dp}$ \\
\hline Empírica & 0.190 & 0.243 & 0.395 & 0.591 & 0.774 & 0.886 & 0.940 & 0.578 & 0.234 \\
Beta & 0.166 & 0.243 & 0.406 & 0.610 & 0.789 & 0.901 & 0.943 & 0.589 & 0.241 \\
LSL & 0.196 & 0.259 & 0.390 & 0.576 & 0.774 & 0.907 & 0.954 & 0.578 & 0.236 \\
LST & 0.190 & 0.261 & 0.402 & 0.582 & 0.763 & 0.895 & 0.948 & 0.576 & 0.232 \\
\hline
\end{tabular}


Tabela 4.27: Critérios de seleção de modelos, Saneamento; $n=181$, ano $=2015$.

\begin{tabular}{l|rrrrrl}
\hline Dist & AIC & BIC & $w_{\text {AIC }}$ & $w_{\text {BIC }}$ & $V_{R V}$ & $p_{V}$ \\
\hline Beta & -198.479 & -192.082 & 0.000 & 0.000 & & \\
Kuma & -198.640 & -192.243 & 0.000 & 0.000 & 1.929 & $0.027^{* *}$ \\
Simplex & -109.669 & -103.272 & 0.000 & 0.000 & -4.606 & 1.000 \\
Gu & -197.912 & -191.515 & 0.000 & 0.000 & -1.685 & 0.954 \\
BR & -201.262 & -191.666 & 0.000 & 0.000 & 0.842 & 0.200 \\
LL & -194.059 & -187.662 & 0.000 & 0.000 & -0.846 & 0.801 \\
LN & -202.550 & -196.153 & 0.000 & 0.001 & 0.680 & 0.248 \\
LT & -202.550 & -190.955 & 0.000 & 0.000 & 0.680 & 0.248 \\
LEP & -219.470 & -209.874 & $\underline{0.912}$ & $\underline{0.979}$ & 1.427 & $0.077^{*}$ \\
LSL & -201.275 & -191.680 & 0.000 & 0.000 & $\underline{3.728}$ & $\underline{0.000^{* * *}}$ \\
LSN & -200.550 & -190.955 & 0.000 & 0.000 & 0.680 & 0.248 \\
LST & -198.738 & -185.944 & 0.000 & 0.000 & 0.711 & 0.239 \\
LSEP & -214.786 & -201.992 & 0.088 & 0.019 & 1.811 & $0.035^{* *}$ \\
\hline
\end{tabular}

$*=$ Rej. $10 \%,{ }^{* *}=$ Rej. $5 \%$ e $^{* * *}=$ Rej. $1 \%$.

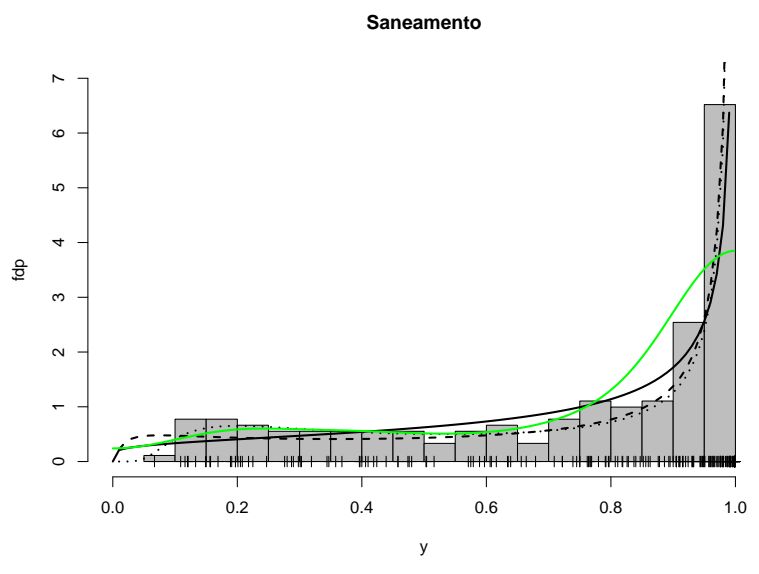

(a) fdp

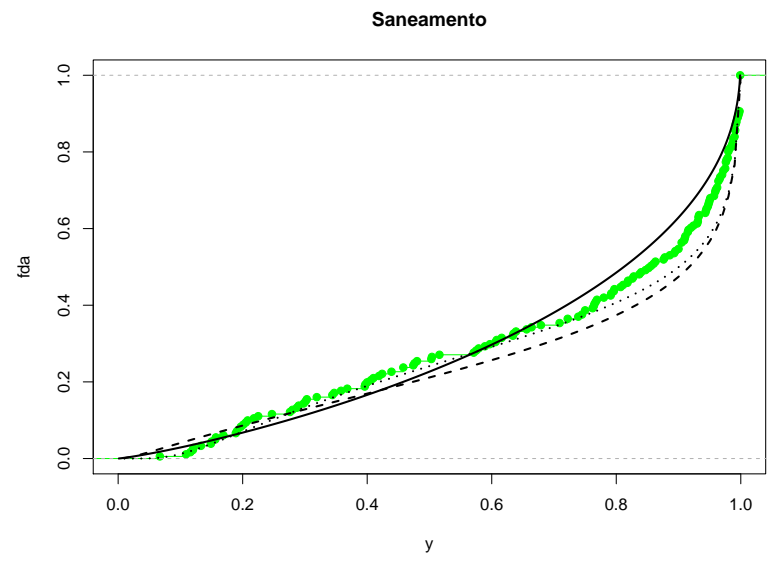

(b) fda

Figura 4.14: Fdp e fda ajustadas, beta (continua), LEP (tracejada) e LSEP (pontilhada), suavização e fda empírica (verde); Saneamento.

Tabela 4.28: Medidas descritivas e estimativas, Saneamento.

\begin{tabular}{l|ccccccccc}
\hline Dist. & $y_{0.05}$ & $y_{0.10}$ & $y_{0.25}$ & $y_{0.50}$ & $y_{0.75}$ & $y_{0.90}$ & $y_{0.95}$ & média & $\mathrm{dp}$ \\
\hline Empírica & 0.157 & 0.218 & 0.480 & 0.856 & 0.972 & 0.997 & 0.999 & 0.727 & 0.292 \\
Beta & 0.157 & 0.273 & 0.535 & 0.813 & 0.955 & 0.991 & 0.997 & 0.730 & 0.274 \\
LEP & 0.120 & 0.233 & 0.586 & 0.917 & 0.989 & 0.998 & 0.999 & 0.764 & 0.294 \\
LSEP & 0.169 & 0.246 & 0.518 & 0.901 & 0.988 & 0.997 & 0.998 & 0.739 & 0.303 \\
\hline
\end{tabular}


Tabela 4.29: Critérios de seleção de modelos, Saneamento urbano; $n=182$, ano $=2015$.

\begin{tabular}{l|rrrrrl}
\hline Dist & AIC & BIC & $w_{\text {AIC }}$ & $w_{\text {BIC }}$ & $V_{R V}$ & $p_{V}$ \\
\hline Beta & -274.078 & -267.670 & 0.000 & 0.000 & & \\
Kuma & -274.248 & -267.840 & 0.000 & 0.000 & 0.638 & 0.262 \\
Simplex & -184.687 & -178.279 & 0.000 & 0.000 & -4.685 & 1.000 \\
Gu & -273.903 & -267.495 & 0.000 & 0.000 & -1.117 & 0.868 \\
BR & -272.078 & -262.466 & 0.000 & 0.000 & 0.000 & 0.500 \\
LL & -267.945 & -261.537 & 0.000 & 0.000 & -1.005 & 0.842 \\
LN & -276.036 & -269.628 & 0.000 & 0.000 & 0.295 & 0.384 \\
LT & -276.036 & -264.424 & 0.000 & 0.000 & 0.295 & 0.384 \\
LEP & -310.696 & -301.084 & $\underline{1.000}$ & $\underline{1.000}$ & $\underline{2.225}$ & $\underline{0.013^{* *}}$ \\
LSL & -275.257 & -265.645 & 0.000 & 0.000 & 1.659 & $0.049^{* *}$ \\
LSN & -274.036 & -264.424 & 0.000 & 0.000 & 0.295 & 0.384 \\
LST & -272.285 & -259.469 & 0.000 & 0.000 & 0.331 & 0.370 \\
LSEP & -287.993 & -275.177 & 0.000 & 0.000 & 1.654 & $0.049^{* *}$ \\
\hline$*$ Rej. $10 \%, * *=$ Rej. $5 \%$ e ${ }^{* * *}=$ Rej. $1 \%$. & & &
\end{tabular}

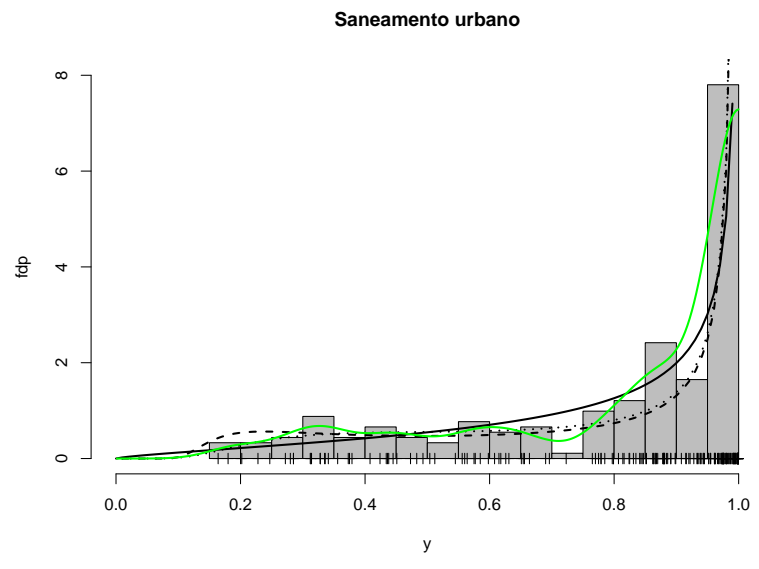

(a) fdp

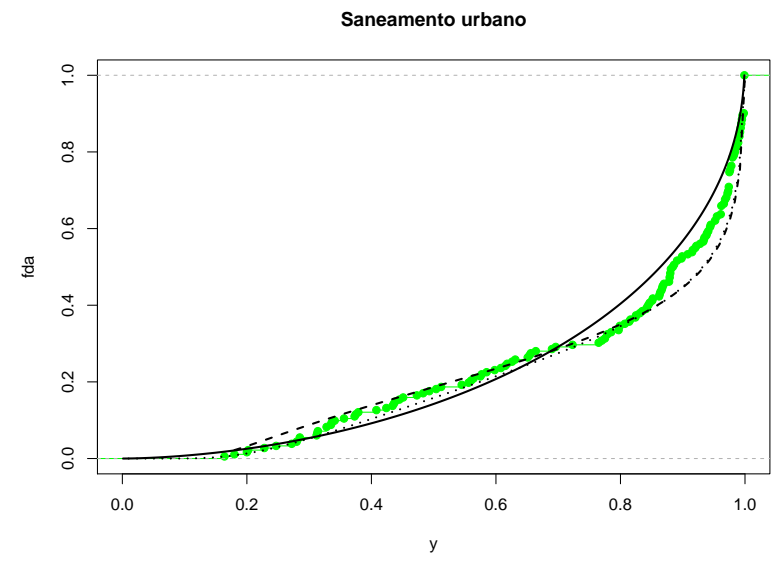

(b) fda

Figura 4.15: Fdp e fda ajustadas, beta (contínua), LEP (tracejada) e LSEP (pontilhada), suavização e fda empírica (verde); Saneamento urbano.

Tabela 4.30: Medidas descritivas e estimativas, Saneamento urbano.

\begin{tabular}{l|ccccccccc}
\hline Dist. & $y_{0.05}$ & $y_{0.10}$ & $y_{0.25}$ & $y_{0.50}$ & $y_{0.75}$ & $y_{0.90}$ & $y_{0.95}$ & média & $\mathrm{dp}$ \\
\hline Empírica & 0.286 & 0.358 & 0.627 & 0.885 & 0.976 & 0.998 & 0.999 & 0.789 & 0.243 \\
Beta & 0.290 & 0.418 & 0.654 & 0.865 & 0.966 & 0.993 & 0.997 & 0.789 & 0.229 \\
LEP & 0.232 & 0.323 & 0.631 & 0.934 & 0.992 & 0.998 & 0.999 & 0.790 & 0.263 \\
LSEP & 0.296 & 0.394 & 0.660 & 0.932 & 0.990 & 0.997 & 0.999 & 0.801 & 0.257 \\
\hline
\end{tabular}


Tabela 4.31: Critérios de seleção de modelos, Títulos da dívida externa; $n=110$, ano $=2015$.

\begin{tabular}{l|rrllll}
\hline Dist & AIC & BIC & $w_{\text {AIC }}$ & $w_{\text {BIC }}$ & $V_{R V}$ & $p_{V}$ \\
\hline Beta & -1.140 & 4.261 & 0.000 & 0.000 & & \\
Kuma & -0.897 & 4.504 & 0.000 & 0.000 & -0.773 & 0.780 \\
Simplex & 210.252 & 215.653 & 0.000 & 0.000 & -8.761 & 1.000 \\
Gu & -0.378 & 5.023 & 0.000 & 0.000 & -1.095 & 0.863 \\
BR & -12.423 & -4.321 & 0.000 & 0.000 & 3.158 & $0.001^{* * *}$ \\
LL & 0.542 & 5.943 & 0.000 & 0.000 & -0.319 & 0.625 \\
LN & 25.513 & 30.914 & 0.000 & 0.000 & -8.079 & 1.000 \\
LT & -35.339 & -25.238 & 0.025 & 0.035 & 3.201 & $0.001^{* * *}$ \\
LEP & -30.181 & -22.080 & 0.002 & 0.007 & 2.873 & $0.002^{* *}$ \\
LSL & -10.486 & -2.385 & 0.000 & 0.000 & 1.113 & 0.133 \\
LSN & 27.514 & 35.615 & 0.000 & 0.000 & -8.103 & 1.000 \\
LST & -40.813 & -30.011 & 0.386 & 0.380 & 3.690 & $0.000^{* * *}$ \\
LSEP & -41.649 & -30.847 & $\underline{0.587}$ & $\underline{0.578}$ & $\underline{3.884}$ & $\underline{0.000^{* * *}}$ \\
\hline
\end{tabular}

$*=$ Rej. $10 \%,{ }^{* *}=$ Rej. $5 \% \mathrm{e}^{* * *}=$ Rej. $1 \%$.

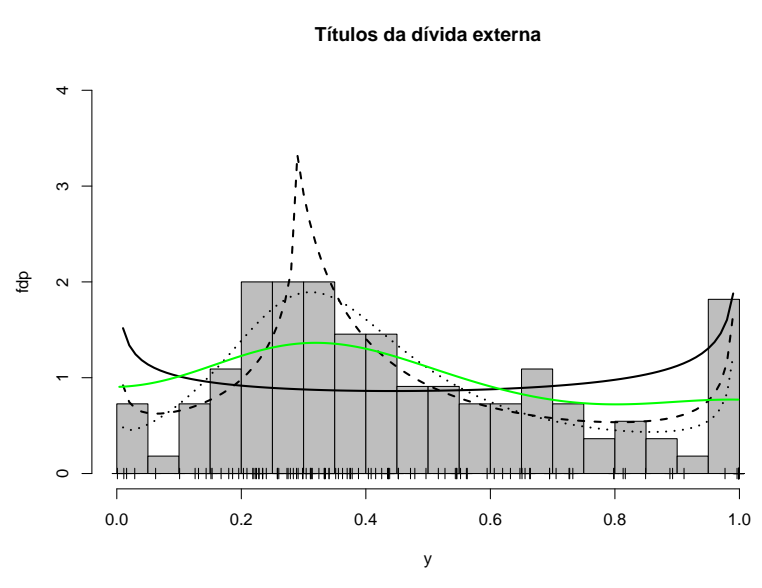

(a) fdp

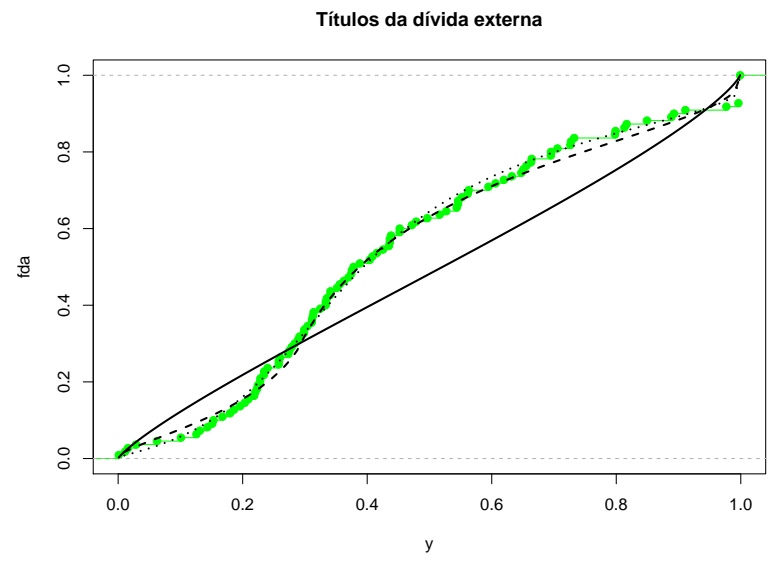

(b) fda

Figura 4.16: Fdp e fda ajustadas, beta (continua), LSEP (tracejada) e LST (pontilhada), suavização e fda empírica (verde); Títulos da divida externa.

Tabela 4.32: Medidas descritivas e estimativas, Títulos da dívida externa.

\begin{tabular}{l|ccccccccc}
\hline Dist. & $y_{0.05}$ & $y_{0.10}$ & $y_{0.25}$ & $y_{0.50}$ & $y_{0.75}$ & $y_{0.90}$ & $y_{0.95}$ & média & $\mathrm{dp}$ \\
\hline Empírica & 0.112 & 0.166 & 0.258 & 0.383 & 0.650 & 0.894 & 0.999 & 0.460 & 0.271 \\
Beta & 0.035 & 0.079 & 0.235 & 0.521 & 0.797 & 0.936 & 0.973 & 0.515 & 0.311 \\
LSEP & 0.058 & 0.134 & 0.273 & 0.387 & 0.661 & 0.926 & 0.985 & 0.464 & 0.276 \\
LST & 0.090 & 0.149 & 0.256 & 0.394 & 0.620 & 0.916 & 0.993 & 0.464 & 0.276 \\
\hline
\end{tabular}


Na Tabela 4.33 apresentamos um resumo informando o número de vezes que cada distribuição foi considerada melhor sob os critérios $w_{\text {AIC }}, w_{\text {BIC }}$ e TRVG.

Tabela 4.33: Resumo dos ajustes.

\begin{tabular}{l|cccccccccccccc}
\hline & \multicolumn{7}{|c|}{ dois parâmetros } & \multicolumn{4}{c|}{ três parâmetros } & \multicolumn{2}{c}{ quatro parâmetros } \\
\hline & Beta & Kuma & Simplex & Gu & BR & LL & LN & LT & LEP & LSL & LSN & LST & LSEP \\
\hline$w_{\text {AIC }}$ & 2 & 1 & 1 & 2 & 1 & 0 & 0 & 1 & 2 & 3 & 0 & 0 & 3 \\
\hline$w_{\text {BIC }}$ & 3 & 1 & 1 & 2 & 2 & 1 & 0 & 0 & 2 & 3 & 0 & 0 & 1 \\
\hline Rej. a 10\% & - & 4 & 0 & 2 & 3 & 0 & 0 & 1 & 3 & 8 & 0 & 6 & 4 \\
\hline Rej. a $5 \%$ & - & 3 & 0 & 1 & 2 & 0 & 0 & 1 & 2 & 4 & 0 & 2 & 4 \\
\hline Rej. a 1\% & - & 0 & 0 & 1 & 2 & 0 & 0 & 1 & 0 & 1 & 0 & 1 & 1 \\
\hline
\end{tabular}

Para seis variáveis (exportações de alta tecnologia, despesas em educação, terra arável, serviços, despesas em pesquisa e desenvolvimento e taxa de alfabetização) entre as dezesseis consideradas, os modelos selecionados são todos de apenas dois parâmetros, a saber, beta, Kumaraswamy, simplex e gama unitário, exceto para a variável exportações de alta tecnologia para a qual a distribuição beta retangular, de três parâmetros, é a terceira selecionada. Entretanto, neste caso, a estimativa de $\alpha$, parâmetro de cauda, é muito próximo de zero (zero até a oitava casa decimal), o que significa que o ajuste é praticamente equivalente ao do modelo beta, de apenas dois parâmetros. Observa-se que as outras distribuições de dois parâmetros consideradas no estudo, ou seja, logito logística e logito normal, não foram selecionadas para modelagem de nenhuma destas variáveis. É notável que o modelo beta não é rejeitado em favor dos modelos alternativos para nenhuma dessas seis variáveis. Ainda, as estimativas produzidas por esses modelos são todas muito próximas. Note-se que tanto as fdp's quanto as fda's ajustadas são semelhantes exceto possivelmente para o ajuste da distribuição Kumaraswamy para a variável despesas em educação e o da distribuição gama unitária para a variável despesas em pesquisa e desenvolvimento. Para o conjunto das seis variáveis as densidades ajustadas apresentam as formas J, J invertido e unimodal, e visualmente produzem ajustes adequados. A escolha de um único modelo para representar cada uma dessas variáveis pode ser feita pelo pesquisador levando em consideração a necessidade ou não de interpretação de parâmetros. Por exemplo, como já mencionado, $\mu$ representa a média das distribuições beta, simplex e gama unitária, e estas distribuições não têm quantis e fda em forma fechada, ao contrário da distribuição Kumaraswamy.

Para a variável energia alternativa e nuclear, o modelo considerado mais adequado é o beta retangular. Note que os pesos $w_{\mathrm{AIC}}$ e $w_{\mathrm{BIC}}$ são bem altos e que o modelo beta retangular acompanha melhor a fda empírica (Figura 4.7(b)). Entretanto, a Tabela 4.14 evidencia que o modelo gama unitário fornece estimativas tão boas quanto o modelo beta retangular. De fato, os pesos baseados em BIC dos modelos beta retangular e gama unitário são bem próximos. Pelo TRVG o modelo beta é rejeitado em favor dos modelos Kumaraswamy, gama unitário, beta retangular e logito skew logística. O modelo simplex não foi considerado já que não houve convergência no processo de estimação dos parâmetros.

Para as demais nove variáveis pelo menos uma distribuição gerada por transformação foi selecionada, sendo de apenas dois parâmetros em um único caso (distribuição logito logística para a variável despesas). Entre as distribuições de três parâmetros a que aparece entre as selecionadas com maior frequência é a logito skew logística, para quatro variáveis. As distribuições de quatro parâmetros, logito skew t e logito skew exponencial potência, aparecem entre as selecionadas três e cinco vezes, respectivamente. 
Observamos visualmente e pelas estimativas de quantis que distribuições por transformação podem, de fato, produzir melhores ajustes do que a distribuição beta. Por exemplo, para a variável exportações de combustível, o modelo logito skew exponencial potência, de quatro parâmetros, parece ser muito mais adequado do que o modelo beta (ver Figura 4.9(b) e Tabela 4.18). Observe, entretanto, que o modelo LSEP aparece entre os selecionados para a modelagem da variável títulos da dívida externa, porém a densidade ajustada não parece compat 'veil com o histograma dos dados. Neste caso, o modelo logito skew t parece fornecer um ajuste mais adequado. 


\section{Capítulo 5}

\section{Considerações Finais}

Nesta dissertação estudamos algumas distribuições de probabilidade contínuas com suporte no intervalo unitário, sendo que a mais comumente utilizada na prática é a distribuição beta. Estudamos também uma classe de distribuições pouco explorada, focando principalmente em duas subclasses. Para um resumo dessas distribuições clique aqui ${ }^{1}$. Nestas subclasses de distribuições é possível obter distribuições flexíveis, que são obtidas por transformações de distribuições flexíveis na reta real (Jones, 2015; Ley, 2015).

Modelamos dados de dezesseis variáveis que representam características de países usando treze diferentes distribuições. Notamos que para diversas variáveis o modelo beta não foi descartado pelo TRVG. Quando rejeitado, em geral o modelo alternativo é um modelo obtido por transformação. Dentre os modelos com dois parâmetros, apenas os modelos Kumaraswamy e gama unitário apresentaram ser alternativas ao modelo beta pelo TRVG. Nos casos em que os modelos ajustados Kumaraswamy e beta foram comparados, as estimativas de quantis e as fda's mostraram-se similares para os dois modelos.

No nosso estudo os modelos logito normal e logito skew normal não foram selecionados entre os mais adequados para modelar nenhuma das variáveis. Os modelos simplex e logito logístico (de dois parâmetros) e o modelo logito t (de três parâmetros) foram raramente selecionados. Devemos destacar que o modelo simplex produziu ajustes consideravelmente piores que os demais modelos para diversas variáveis. Além disso, o processo de estimação sob o modelo simplex apresentou problemas de convergência em algumas situações e mostrou-se muito sensível à escolha de valores iniciais.

Entre os modelos obtidos por transformações, notamos que aqueles que envolvem distribuições skew, que contemplam um parâmetro de assimetria, são bem mais flexíveis do que os que são baseados em distribuições simétricas.

O pacote gamlss é útil para ajuste de distribuições obtidas por transformação logística. A função gen.Family () permite que qualquer distribuição com suporte na reta real disponível no pacote (por exemplo, t e skew t) seja transformada através da transformação logística para uma nova família de distribuições com suporte no intervalo unitário (por exemplo, logito t e logito skew t); ver Hossain et al. (2016, Seção 2.3). Implementamos um código em $\mathrm{R}$ e uma página na internet para analisar dados no intervalo unitário, tendo como banco de dados default o apresentado nesta dissertação. Para acessar clique aqui ${ }^{2}$.

\footnotetext{
${ }^{1}$ https://francimario.shinyapps.io/unit_data/\#section-introduction

${ }^{2}$ https://francimario.shinyapps.io/unit_data/\#section-fit
} 
Naturais e possíveis extensões deste trabalho são: comparação dos modelos estudados com modelos obtidos por mistura de distribuições beta, estudos de modelos logito e logito skew inflados de zero e/ou um e estudo de modelos de regressão logito skew com correspondente análise de diagnóstico e implementação computacional. 


\section{Referências Bibliográficas}

Akaike(1973) Hirotogu Akaike. Information theory and an extension of the maximum likelihood principle. Second International Symposium on Information Theory, 1:199-213. Citado na pág. 27

Anderson e Fang(1990) Theodore Wilbur Anderson e Kai-Tai Fang. Statistical Inference in Elliptically Contoured and Related Distributions. Allerton Press, New York. Citado na pág. 11

Azzalini(1985) Adelchi Azzalini. A class of distributions which includes the normal ones. Scandinavian Journal of Statistics, 12:171-178. Citado na pág. 11, 20

Azzalini(1986) Adelchi Azzalini. Further results on a class of distributions which includes the normal ones. Statistica, 46:199-208. Citado na pág. 23

Azzalini(2017) Adelchi Azzalini. Package "sn". Citado na pág. 25

Azzalini e Capitanio(2003) Adelchi Azzalini e Antonella Capitanio. Distributions generated by perturbation of symmetry with emphasis on a multivariate skew t-distribution. Journal of the Royal Statistical Society: Series B (Statistical Methodology), 65:367-389. Citado na pág. 21

Barndorff-Nielsen e Jørgensen(1991) Ole E Barndorff-Nielsen e Bent Jørgensen. Some parametric models on the simplex. Journal of Multivariate Analysis, 39:106-116. Citado na pág. 7

Bateman et al.(1953) Harry Bateman, Arthur Erdélyi et al. Higher Transcendental Functions. McGraw-Hill, New York. Citado na pág. 17

Bayes et al.(2012) Cristian L Bayes, Jorge L Bazán, Catalina García et al. A new robust regression model for proportions. Bayesian Analysis, 7:841-866. Citado na pág. 9

Burnham e Anderson(2002) Kenneth P Burnham e David R Anderson. Model Selection and Multimodel Inference: A Practical Information-Theoretic Approach. Springer Science \& Business Media, New York. Citado na pág. 28

DiCiccio e Monti(2004) Thomas J DiCiccio e Anna Clara Monti. Inferential aspects of the skew exponential power distribution. Journal of the American Statistical Association, 99:439-450. Citado na pág. 23

Ferrari e Cribari-Neto(2004) Silvia LP Ferrari e Francisco Cribari-Neto. Beta regression for modelling rates and proportions. Journal of Applied Statistics, 31:799-815. Citado na pág. 1,4 
García et al.(2011) CB García, J García Pérez e J René van Dorp. Modeling heavy-tailed, skewed and peaked uncertainty phenomena with bounded support. Statistical Methods $\&$ Applications, 20:463-486. Citado na pág. 8

Grassia(1977) A Grassia. On a family of distributions with argument between 0 and 1 obtained by transformation of the gamma and derived compound distributions. Australian \& New Zealand Journal of Statistics, 19:108-114. Citado na pág. 7

Gupta et al.(2002) AK Gupta, Fu-Chuen Chang e Wen-Jang Huang. Some skew-symmetric models. Random Operators and Stochastic Equations, 10:133-140. Citado na pág. 11, 19, 20, 21

Gupta e Nadarajah(2004) Arjun K Gupta e Saralees Nadarajah. Handbook of Beta Distribution and its Applications. CRC press. Citado na pág. 1

Hahn(2008) Eugene David Hahn. Mixture densities for project management activity times: A robust approach to pert. European Journal of Operational Research, 188:450-459. Citado na pág. 8

Hossain et al.(2016) Abu Hossain, Robert Rigby, Mikis Stasinopoulos e Marco Enea. Centile estimation for a proportion response variable. Statistics in Medicine, 35:895-904. Citado na pág. 21,51

Johnson(1949) Norman L Johnson. Systems of frequency curves generated by methods of translation. Biometrika, (1/2):149-176. Citado na pág. 12, 14

Jones(2015) MC Jones. On families of distributions with shape parameters. International Statistical Review, 83:175-192. Citado na pág. 1, 3, 51

Kotz e Van Dorp(2004) Samuel Kotz e Johan Ren Van Dorp. Beyond Beta: Other Continuous Families of Distributions with Bounded Support and Applications. World Scientific, Hackensack. Citado na pág. 3

Kumaraswamy(1980) Ponnambalam Kumaraswamy. A generalized probability density function for double-bounded random processes. Journal of Hydrology, 46:79-88. Citado na pág. 1,4

Lemonte e Bazán(2015) Artur J Lemonte e Jorge L Bazán. New class of johnson sb distributions and its associated regression model for rates and proportions. Biometrical Journal. Citado na pág. iii, 2, 14, 24

Ley(2015) Christophe Ley. Flexible modelling in statistics: past, present and future. Journal de la Société Franccaise de Statistique, 156:76-96. Citado na pág. 1, 51

Mitnik e Baek(2013) Pablo A Mitnik e Sunyoung Baek. The kumaraswamy distribution: median-dispersion re-parameterizations for regression modeling and simulation-based estimation. Statistical Papers, 54:177-192. Citado na pág. 5, 6

Nadarajah(2009) Saralees Nadarajah. The skew logistic distribution. AStA Advances in Statistical Analysis, 93:187-203. Citado na pág. 19

R Core Team(2017) R Core Team. R: A Language and Environment for Statistical Computing. R Foundation for Statistical Computing, Vienna, Austria, 2017. URL https://www.R-project.org/. Citado na pág. 27, 29 
Rennolls e Wang(2005) Keith Rennolls e Mingliang Wang. A new parameterization of johnson's sb distribution with application to fitting forest tree diameter data. Canadian Journal of Forest Research, 35:575-579. Citado na pág. 14

Rigby e Stasinopoulos(2005) Robert A Rigby e D Mikis Stasinopoulos. Generalized additive models for location, scale and shape. Journal of the Royal Statistical Society: Series C (Applied Statistics), 54:507-554. Citado na pág. 14

Schwarz(1978) Gideon Schwarz. Estimating the dimension of a model. The Annals of Statistics, 6:461-464. Citado na pág. 28

Smithson e Merkle(2013) Michael Smithson e Edgar C Merkle. Generalized Linear Models for Categorical and Continuous Limited Dependent Variables. CRC Press, New York. Citado na pág. iii, 1, 2, 11, 12

Stasinopoulos et al.(2017) Mikis Stasinopoulos, Bob Rigby, Vlasios Voudouris, Calliope Akantziliotou, Marco Enea e Daniil Kiose. Package "gamlss". Citado na pág. 14, 27

Tadikamalla e Johnson(1982) Pandu R Tadikamalla e Norman L Johnson. Systems of frequency curves generated by transformations of logistic variables. Biometrika, 69: 461-465. Citado na pág. 12, 14

Vuong(1989) Quang H Vuong. Likelihood ratio tests for model selection and non-nested hypotheses. Econometrica: Journal of the Econometric Society, 57:307-333. Citado na pág. 28,29

World Bank(2017) The World Bank. World bank open data, 2017. URL https://data. worldbank.org/. Citado na pág. 29 